\title{
Higgs boson masses and $B$-physics constraints in Non-Minimal Flavor Violating SUSY scenarios
}

\author{
M. Arana-Catania, ${ }^{a}$ S. Heinemeyer, ${ }^{b}$ M.J. Herrero ${ }^{a}$ and S. Peñaranda ${ }^{c}$ \\ ${ }^{a}$ Departamento de Física Teórica and Instituto de Física Teórica, \\ IFT-UAM/CSIC, Universidad Autónoma de Madrid, \\ Nicolás Cabrera 13-15, Cantoblanco, E-28049 Madrid, Spain \\ ${ }^{b}$ Instituto de Física de Cantabria (IFCA-UC/CSIC), \\ Avda. de los Castros s/n, E-39005 Santander, Spain \\ ${ }^{c}$ Departamento de Física Teórica, Universidad de Zaragoza, \\ Pedro Cerbuna 12, E-50009 Zaragoza, Spain \\ E-mail: miguel.arana@uam.es, Sven.Heinemeyer@cern.ch, \\ Maria.Herrero@uam.es, siannah@unizar.es
}

ABSTRaCT: We present one-loop corrections to the Higgs boson masses in the MSSM with Non-Minimal Flavor Violation. The flavor violation is generated from the hypothesis of general flavor mixing in the squark mass matrices, and these are parametrized by a complete set of $\delta_{i j}^{X Y}(X, Y=L, R ; i, j=t, c, u$ or $b, s, d)$. We calculate the corrections to the Higgs masses in terms of these $\delta_{i j}^{X Y}$ taking into account all relevant restrictions from $B$-physics data. This includes constraints from $\operatorname{BR}\left(B \rightarrow X_{s} \gamma\right), \operatorname{BR}\left(B_{s} \rightarrow \mu^{+} \mu^{-}\right)$and $\Delta M_{B_{s}}$. After taking into account these constraints we find sizable corrections to the Higgs boson masses, in the case of the lightest MSSM Higgs boson mass exceeding tens of GeV. These corrections are found mainly for the low $\tan \beta$ case. In the case of a Higgs boson mass measurement these corrections might be used to set further constraints on $\delta_{i j}^{X Y}$.

KeYWORDs: Supersymmetry Phenomenology

ARXIV EPRINT: 1109.6232 


\section{Contents}

1 Introduction 1

2 SUSY scenarios with Non-Minimal Flavor Violation 3

3 Constraints on Non-Minimal Flavor Violating SUSY scenarios from $B$ physics

$3.1 \quad \mathrm{BR}\left(B \rightarrow X_{s} \gamma\right) \quad 9$

$3.2 \operatorname{BR}\left(B_{s} \rightarrow \mu^{+} \mu^{-}\right) \quad 11$

$\begin{array}{lll}3.3 \Delta M_{B_{s}} & 12\end{array}$

$\begin{array}{lll}3.4 & \text { Numerical results on } B \text { observables } & 14\end{array}$

4 Radiative corrections to MSSM Higgs masses within NMFV scenarios 21

4.1 The Higgs boson sector at tree-level 21

$\begin{array}{ll}4.2 & \text { The Higgs boson sector at one-loop } \\ 25\end{array}$

4.3 Analytical results of Higgs mass corrections in NMFV-SUSY 27

5 Numerical analysis $\quad \mathbf{2 8}$

$5.1 \Delta m_{\phi}$ versus one $\delta_{i j}^{X Y} \neq 0 \quad 28$

$5.2 \Delta m_{h}$ versus two $\delta_{i j}^{X Y} \neq 0 \quad 29$

6 Conclusions $\quad 36$

$\begin{array}{ll}\text { A Feynman rules } & 40\end{array}$

B Tadpoles and self-energies $\quad 44$

\section{Introduction}

The Higgs sector of the Minimal Supersymmetric Standard Model (MSSM) [1-3] with two scalar doublets accommodates five physical Higgs bosons. In lowest order these are the light and heavy $\mathcal{C P}$-even $h$ and $H$, the $\mathcal{C P}$-odd $A$, and the charged Higgs bosons $H^{ \pm}$. The Higgs sector of the MSSM can be expressed at lowest order in terms of the gauge couplings, $M_{A}$ and $\tan \beta \equiv v_{2} / v_{1}$, the ratio of the two vacuum expectation values. All other masses and mixing angles can therefore be predicted. Higher-order contributions can give large corrections to the tree-level relations (see e.g. ref. [4, 5] for reviews).

The MSSM predicts scalar partners for each fermionic degree of freedom in the Standard Model (SM), and fermionic partners for Higgs and gauge bosons. So far, the direct search for SUSY particles has not been successful. One can only set lower bounds of several hundreds of $\mathrm{GeV}$, depending on the particle and the model specifications [6, 7]. To lift 
the masses of the SUSY partners from the corresponding SM values, soft SUSY-breaking terms are introduced [1-3]. The most general flavor structure of the soft SUSY-breaking sector with flavor non-diagonal terms would induce large flavor-changing neutral-currents, contradicting the experimental results [8]. Attempts to avoid this kind of problem include flavor-blind SUSY-breaking scenarios, like minimal Supergravity or gauge-mediated SUSYbreaking. In these scenarios, the sfermion-mass matrices are flavor diagonal in the same basis as the quark matrices at the SUSY-breaking scale. However, a certain amount of flavor mixing is generated due to the renormalization-group evolution from the SUSY-breaking scale down to the electroweak scale. In a more agnostic approach all flavor-violating terms are introduced as free parameters, and each model point, i.e. each combination of flavorviolating parameters, is tested against experimental data.

Similarly strong bounds exist for the MSSM Higgs sector from the non-observation of Higgs bosons at LEP $[9,10]$, the Tevatron ${ }^{1}$ and most recently from LHC searches [12]. The LHC has good prospects to discover at least one neutral Higgs boson over the full MSSM parameter space. A precision on the mass of a SM-like Higgs boson of $\sim 200 \mathrm{MeV}$ is expected [13-15]. At the ILC a determination of the Higgs boson properties (within the kinematic reach) will be possible, and an accuracy on the mass could reach the $50 \mathrm{MeV}$ level [1619]. The interplay of the LHC and the ILC in the neutral MSSM Higgs sector is discussed in ref. [20, 21].

For the $\mathrm{MSSM}^{2}$ the status of higher-order corrections to the masses and mixing angles in the neutral Higgs sector is quite advanced. The full one-loop and potentially all leading two-loop corrections are known, see ref. [24] for a review. Most recently leading three-loop corrections became available [25-27].

However, the impact of non-minimal flavor violation (NMFV) on the MSSM Higgsboson masses and mixing angles, entering already at the one-loop level, has not been explored very deeply so far. A one-loop calculation taking into account the $L L$-mixing between the third and second generation of scalar up-type quarks has been performed in ref. [28]. A full one-loop calculation of the Higgs-boson self-energies including all NMFV mixing terms had been implemented into the Fortran code FeynHiggs [23, 24, 29-31], however no cross checks or numerical evaluations analyzing the effects of the new mixing terms were performed. Possible effects from NMFV on Higgs boson decays were investigated in [32-36]. Within a similar context of NMFV there have been also studied some effects of scharm-stop flavor mixing in top-quark FCNC processes [37] and charged Higgs processes [38] as well as the implications for LHC [38, 39]. Some previous studies on the induced radiative corrections on the Higgs mass from scharm-stop flavor mixing have also been performed in $[37,39]$, but any effects from mixing involving the first generation of scalar quarks have been neglected. The numerical estimates in [37, 39] also neglect all the flavor mixings in the scalar down-type sector, except for those of $L L$-type that are induced from the scalar up-type sector by $\mathrm{SU}(2)$ invariance. In [39] they also consider one example with a particular numerical value of non-vanishing $\tilde{s}_{L}-\tilde{b}_{R}$ mixing.

\footnotetext{
${ }^{1}$ See $[11]$ and references therein.

${ }^{2}$ We concentrate here on the case with real parameters. For complex parameters see refs. [22, 23] and references therein.
} 
We study in this paper the consequences from NMFV for the MSSM Higgs-boson spectrum, where our results are obtained in full generality, i.e. all generations in the scalar upand down-type quark sector are included in our analytical results. In the numerical analysis we focus particularly on the flavor mixing between the second and third generations which is the relevant one in $B$ physics. Our estimates include all type of flavor mixings, $L L, L R, R L$, and $R R$. We devote special attention to the $L R / R L$ sector. These kind of mixing effects are expected to be sizable, since they enter the off-diagonal $A$ parameters, which appear directly in the coupling of the Higgs bosons to scalar quarks.

Concerning the constraints from flavor observables we take into account the most up-to-date evaluations in the NMFV MSSM for $\operatorname{BR}\left(B \rightarrow X_{s} \gamma\right),{ }^{3} \operatorname{BR}\left(B_{s} \rightarrow \mu^{+} \mu^{-}\right)$and $\Delta M_{B_{s}}$, based on the BPHYSICS subroutine included in the SuFla code. ${ }^{4}$ For the evaluation of $\Delta M_{B_{s}}$ we have incorporated into this subroutine additional contributions from the one-loop gluino boxes [44] which are known to be very relevant in the context of NMFV scenarios $[45-47]$.

In the first step of the analysis we scan over the NMFV parameters, and contrast them with the experimental bounds on $\mathrm{BR}\left(B \rightarrow X_{s} \gamma\right), \mathrm{BR}\left(B_{s} \rightarrow \mu^{+} \mu^{-}\right)$and $\Delta M_{B_{s}}$. In the second step we analyze the one-loop contributions of NMFV to the MSSM Higgs boson masses, focusing on the parameter space still allowed by the experimental flavor constraints. In this way the full possible impact of NMFV in the MSSM on the Higgs sector can be explored. The paper is organized as follows: in section 2 we introduce our notation for the NMFV MSSM and define certain benchmark scenarios that are used for the subsequent analysis. Our implementation and new results on $B$-physics observables is given in section 3, where we also analyze in detail which combination of NMFV parameters are still allowed by current experimental constraints. The calculation of the corrections to Higgs boson masses in the NMFV MSSM is presented in section 4. The numerical analysis of the impact of the one-loop Higgs mass corrections is given in section 5. Our conclusions can be found in section 6 .

\section{SUSY scenarios with Non-Minimal Flavor Violation}

We work in SUSY scenarios with the same particle content as the MSSM, but with general flavor mixing hypothesis in the squark sector. Within these SUSY-NMFV scenarios, besides the usual flavor violation originated by the CKM matrix of the quark sector, the general flavor mixing in the squark mass matrices additionally generates flavor violation from the squark sector. These squark flavor mixings are usually described in terms of a set of dimensionless parameters $\delta_{i j}^{X Y}(X, Y=L, R ; i, j=t, c, u$ or $b, s, d)$, which for simplicity in the computations are frequently considered within the Mass Insertion Approximation (MIA) [48]. We will not use here this approximation, but instead we will solve exactly the diagonalization of the squark mass matrices.

\footnotetext{
${ }^{3}$ Subleading NLO MSSM corrections were evaluated in $[40,41]$. However, their effect on our evaluations would be minor.

${ }^{4}$ See $[42,43]$ and references therein.
} 
In this section we summarize the main features of the squark flavor mixing within the SUSY-NMFV scenarios and set the notation.

The relevant MSSM superpotential terms are:

$$
W=\epsilon_{\alpha \beta}\left[\hat{H}_{2}^{\alpha} \hat{Q}^{\beta} Y^{u} \hat{U}-\hat{H}_{1}^{\alpha} \hat{Q}^{\beta} Y^{d} \hat{D}+\mu \hat{H}_{1}^{\alpha} \hat{H}_{2}^{\beta}\right],
$$

where the involved superfields are: $\hat{Q}$, containing the quark $\left(\begin{array}{lll}u_{L} & d_{L}\end{array}\right)^{T}$ and squark $\left(\tilde{u}_{L} \tilde{d}_{L}\right)^{T}$ $\mathrm{SU}(2)$ doublets; $\hat{U}$, containing the quark $\left(u_{R}\right)^{c}$ and squark $\tilde{u}_{R}^{*} \mathrm{SU}(2)$ singlets; $\hat{D}$, containing the quark $\left(d_{R}\right)^{c}$ and squark $\tilde{d}_{R}^{*} \mathrm{SU}(2)$ singlets; and $\hat{H}_{1,2}$ containing the Higgs bosons $\mathrm{SU}(2)$ doublets, $\mathcal{H}_{1}=\left(\mathcal{H}_{1}^{0} \mathcal{H}_{1}^{-}\right)^{T}$ and $\mathcal{H}_{2}=\left(\mathcal{H}_{2}^{+} \mathcal{H}_{2}^{0}\right)^{T}$, and their SUSY partners. $f^{c}$ denotes here the particle-antiparticle conjugate of fermion $f$, and $\tilde{f}^{*}$ denotes the complex conjugate of sfermion $\tilde{f}$. We follow the notation of [49], but with the the convention $\epsilon_{12}=-1$. The generation indices in the superfields, $\hat{Q}_{i}, \hat{U}_{i}, \hat{D}_{i}$, quarks $q_{i}$, squarks $\tilde{q}_{i},(i=1,2,3)$, and Yukawa coupling $3 \times 3$ matrices, $Y_{i j}^{u}, Y_{i j}^{d},(i, j=1,2,3)$, have been omitted above for brevity.

Usually one starts rotating the quark fields from the $\mathrm{SU}(2)$ (interaction) eigenstate basis, $q_{L, R}^{\text {int }}$, to the mass (physical), $q_{L, R}^{\text {phys }}$ eigenstate basis by unitary transformations, $V_{L, R}^{u, d}$ :

$$
\left(\begin{array}{c}
u_{L, R}^{\text {phys }} \\
c_{L, R}^{\text {phys }} \\
t_{L, R}^{\text {phys }}
\end{array}\right)=V_{L, R}^{u}\left(\begin{array}{c}
u_{L, R}^{\text {int }} \\
c_{L, R}^{\text {int }} \\
t_{L, R}^{\text {int }}
\end{array}\right), \quad\left(\begin{array}{c}
d_{L, R}^{\text {phys }} \\
s_{L, R}^{\text {phys }} \\
b_{L, R}^{\text {phys }}
\end{array}\right)=V_{L, R}^{d}\left(\begin{array}{c}
d_{L, R}^{\text {int }} \\
s_{L, R}^{\text {int }} \\
b_{L, R}^{\text {int }}
\end{array}\right),
$$

such that the fermion mass matrices in the physical basis are diagonal:

$$
\begin{aligned}
V_{L}^{u} Y^{u *} V_{R}^{u \dagger} & =\operatorname{diag}\left(y_{u}, y_{c}, y_{t}\right)=\operatorname{diag}\left(\frac{m_{u}}{v_{2}}, \frac{m_{c}}{v_{2}}, \frac{m_{t}}{v_{2}}\right), \\
V_{L}^{d} Y^{d *} V_{R}^{d \dagger} & =\operatorname{diag}\left(y_{d}, y_{s}, y_{b}\right)=\operatorname{diag}\left(\frac{m_{d}}{v_{1}}, \frac{m_{s}}{v_{1}}, \frac{m_{b}}{v_{1}}\right),
\end{aligned}
$$

where $v_{1}=\left\langle\mathcal{H}_{1}^{0}\right\rangle$ and $v_{2}=\left\langle\mathcal{H}_{2}^{0}\right\rangle$ are the vacuum expectation values of the neutral Higgs fields. The CKM matrix, which is responsible for the flavor violation in the quark sector, is given as usual as,

$$
V_{\mathrm{CKM}}=V_{L}^{u} V_{L}^{d \dagger}
$$

A simultaneous (parallel) rotation of the squarks with the same above unitary matrices as their corresponding quark partners leads to the so-called Super-CKM basis. In the NMFV scenarios, contrary to the MFV ones, the Super-CKM basis does not coincide with the physical squark basis, i.e, their corresponding squark mass matrices are not yet diagonal. More concretely, rotating the squarks from the interaction basis, $\tilde{q}_{L, R}^{\mathrm{int}}$ to the Super-CKM basis, $\tilde{q}_{L, R}$, by

$$
\left(\begin{array}{c}
\tilde{u}_{L, R} \\
\tilde{c}_{L, R} \\
\tilde{t}_{L, R}
\end{array}\right)=V_{L, R}^{u}\left(\begin{array}{c}
\tilde{u}_{L, R}^{\mathrm{int}} \\
\tilde{c}_{L, R}^{\mathrm{int}} \\
\tilde{t}_{L, R}^{\mathrm{nt}}
\end{array}\right), \quad\left(\begin{array}{c}
\tilde{d}_{L, R} \\
\tilde{s}_{L, R} \\
\tilde{b}_{L, R}
\end{array}\right)=V_{L, R}^{d}\left(\begin{array}{c}
\tilde{d}_{L, R}^{\mathrm{int}} \\
\tilde{s}_{L, R}^{\mathrm{int}} \\
\tilde{b}_{L, R}^{\mathrm{int}}
\end{array}\right),
$$

one gets the soft-SUSY-breaking Lagrangian transformed from:

$$
\begin{aligned}
\mathcal{L}_{\text {soft }}= & -\tilde{\mathcal{U}}_{i}^{\text {int* }} m_{\tilde{U} i j}^{2} \tilde{\mathcal{U}}_{j}^{\text {int }}-\tilde{\mathcal{D}}_{i}^{\text {int* }} m_{\tilde{D} i j}^{2} \tilde{\mathcal{D}}_{j}^{\text {int }}-\tilde{\mathcal{Q}}_{i}^{\text {int }} m_{\tilde{Q}_{i j}}^{2} \tilde{\mathcal{Q}}_{j}^{\text {int }} \\
& -\left[\tilde{\mathcal{Q}}_{i}^{\text {int }} \overline{\mathcal{A}}_{i j}^{u} \tilde{\mathcal{U}}_{j}^{\text {int* }} \mathcal{H}_{2}-\tilde{\mathcal{Q}}_{i}^{\text {int }} \overline{\mathcal{A}}_{i j}^{d} \tilde{\mathcal{D}}_{j}^{\text {int* }} \mathcal{H}_{1}+\text { h.c. }\right]
\end{aligned}
$$


to:

$$
\begin{aligned}
\mathcal{L}_{\text {soft }} & =-\tilde{\mathcal{U}}_{R i}^{*} m_{\tilde{U}_{R} i j}^{2} \tilde{\mathcal{U}}_{R j}-\tilde{\mathcal{D}}_{R i}^{*} m_{\tilde{D}_{R} i j}^{2} \tilde{\mathcal{D}}_{R j}-\tilde{\mathcal{U}}_{L i}^{*} m_{\tilde{U}_{L} i j}^{2} \tilde{\mathcal{U}}_{L j}-\tilde{\mathcal{D}}_{L i}^{*} m_{\tilde{D}_{L} i j}^{2} \tilde{\mathcal{D}}_{L j} \\
- & {\left[\tilde{\mathcal{U}}_{L i} \mathcal{A}_{i j}^{u} \tilde{\mathcal{U}}_{R j}^{*} \mathcal{H}_{2}^{0}-\tilde{\mathcal{D}}_{L i}\left(V_{\mathrm{CKM}}\right)_{k i} \mathcal{A}_{k j}^{u} \tilde{\mathcal{U}}_{R j}^{*} \mathcal{H}_{2}^{+}-\tilde{\mathcal{U}}_{L i}\left(V_{\mathrm{CKM}}^{*}\right)_{i k} \mathcal{A}_{k j}^{d} \tilde{\mathcal{D}}_{R j}^{*} \mathcal{H}_{1}^{-}+\tilde{\mathcal{D}}_{L i} \mathcal{A}_{i j}^{d} \tilde{\mathcal{D}}_{R j}^{*} \mathcal{H}_{1}^{0}+\text { h.c. }\right], }
\end{aligned}
$$

where we have used calligraphic capital letters for the squark fields with generation indexes,

$$
\begin{aligned}
\tilde{\mathcal{U}}_{1,2,3}^{\text {int }} & =\tilde{u}_{R}^{\text {int }}, \tilde{c}_{R}^{\text {int }}, \tilde{t}_{R}^{\text {int }} ; \tilde{\mathcal{D}}_{1,2,3}^{\text {int }}=\tilde{d}_{R}^{\text {int }}, \tilde{s}_{R}^{\text {int }}, \tilde{b}_{R}^{\text {int }} ; \tilde{\mathcal{Q}}_{1,2,3}^{\text {int }}=\left(\tilde{u}_{L}^{\text {int }} \tilde{d}_{L}^{\text {int }}\right)^{T},\left(\tilde{c}_{L}^{\text {int }} \tilde{s}_{L}^{\text {int }}\right)^{T},\left(\tilde{t}_{L}^{\text {int }} \tilde{b}_{L}^{\text {int }}\right)^{T} ; \\
\tilde{\mathcal{U}}_{L 1,2,3} & =\tilde{u}_{L}, \tilde{c}_{L}, \tilde{t}_{L} ; \tilde{\mathcal{D}}_{L 1,2,3}=\tilde{d}_{L}, \tilde{s}_{L}, \tilde{b}_{L} ; \tilde{\mathcal{U}}_{R 1,2,3}=\tilde{u}_{R}, \tilde{c}_{R}, \tilde{t}_{R} ; \tilde{\mathcal{D}}_{R 1,2,3}=\tilde{d}_{R}, \tilde{s}_{R}, \tilde{b}_{R} ;
\end{aligned}
$$

and $(q=u, d)$

$$
\mathcal{A}^{q}=V_{L}^{q} \overline{\mathcal{A}}^{q} V_{R}^{q \dagger}, m_{\tilde{U}_{R}}^{2}=V_{R}^{u} m_{\tilde{U}}^{2} V_{R}^{u \dagger}, m_{\tilde{D}_{R}}^{2}=V_{R}^{d} m_{\tilde{D}}^{2} V_{R}^{d \dagger}, m_{\tilde{U}_{L}}^{2}=V_{L}^{u} m_{\tilde{Q}}^{2} V_{L}^{u \dagger}, m_{\tilde{D}_{L}}^{2}=V_{L}^{d} m_{\tilde{Q}}^{2} V_{L}^{d \dagger} .
$$

The usual procedure to introduce general flavor mixing in the squark sector is to include the non-diagonality in flavor space at this stage, namely, in the Super-CKM basis. Thus, one usually writes the $6 \times 6$ non-diagonal mass matrices, $\mathcal{M}_{\tilde{u}}^{2}$ and $\mathcal{M}_{\tilde{d}}^{2}$, referred to the SuperCKM basis, being ordered respectively as $\left(\tilde{u}_{L}, \tilde{c}_{L}, \tilde{t}_{L}, \tilde{u}_{R}, \tilde{c}_{R}, \tilde{t}_{R}\right)$ and $\left(\tilde{d}_{L}, \tilde{s}_{L}, \tilde{b}_{L}, \tilde{d}_{R}, \tilde{s}_{R}, \tilde{b}_{R}\right)$, and write them in terms of left- and right-handed blocks $M_{\tilde{q} A B}^{2}(q=u, d, A, B=L, R)$, which are non-diagonal $3 \times 3$ matrices,

$$
\mathcal{M}_{\tilde{q}}^{2}=\left(\begin{array}{cc}
M_{\tilde{q} L L}^{2} & M_{\tilde{q} L R}^{2} \\
M_{\tilde{q} L R}^{2 \dagger} & M_{\tilde{q} R R}^{2}
\end{array}\right), \quad \tilde{q}=\tilde{u}, \tilde{d}
$$

where:

$$
\begin{aligned}
M_{\tilde{u} L L i j}^{2} & =m_{\tilde{U}_{L} i j}^{2}+\left(m_{u_{i}}^{2}+\left(T_{3}^{u}-Q_{u} \sin ^{2} \theta_{W}\right) M_{Z}^{2} \cos 2 \beta\right) \delta_{i j}, \\
M_{\tilde{u} R R i j}^{2} & =m_{\tilde{U}_{R} i j}^{2}+\left(m_{u_{i}}^{2}+Q_{u} \sin ^{2} \theta_{W} M_{Z}^{2} \cos 2 \beta\right) \delta_{i j}, \\
M_{\tilde{u} L R i j}^{2} & =\left\langle\mathcal{H}_{2}^{0}\right\rangle \mathcal{A}_{i j}^{u}-m_{u_{i}} \mu \cot \beta \delta_{i j}, \\
M_{\tilde{d} L L i j}^{2} & =m_{\tilde{D}_{L} i j}^{2}+\left(m_{d_{i}}^{2}+\left(T_{3}^{d}-Q_{d} \sin ^{2} \theta_{W}\right) M_{Z}^{2} \cos 2 \beta\right) \delta_{i j}, \\
M_{\tilde{d} R R i j}^{2} & =m_{\tilde{D}_{R} i j}^{2}+\left(m_{d_{i}}^{2}+Q_{d} \sin ^{2} \theta_{W} M_{Z}^{2} \cos 2 \beta\right) \delta_{i j}, \\
M_{\tilde{d} L R i j}^{2} & =\left\langle\mathcal{H}_{1}^{0}\right\rangle \mathcal{A}_{i j}^{d}-m_{d_{i}} \mu \tan \beta \delta_{i j},
\end{aligned}
$$

with, $i, j=1,2,3, Q_{u}=2 / 3, Q_{d}=-1 / 3, T_{3}^{u}=1 / 2$ and $T_{3}^{d}=-1 / 2 . \theta_{W}$ is the weak angle, $M_{Z}$ is the $Z$ gauge boson mass, and $\left(m_{u_{1}}, m_{u_{2}}, m_{u_{3}}\right)=\left(m_{u}, m_{c}, m_{t}\right),\left(m_{d_{1}}, m_{d_{2}}, m_{d_{3}}\right)=$ $\left(m_{d}, m_{s}, m_{b}\right)$. It should be noted that the non-diagonality in flavor comes from the values of $m_{\tilde{U}_{L} i j}^{2}, m_{\tilde{U}_{R} i j}^{2}, m_{\tilde{D}_{L} i j}^{2}, m_{\tilde{D}_{R} i j}^{2}, \mathcal{A}_{i j}^{u}$ and $\mathcal{A}_{i j}^{d}$ for $i \neq j$.

The next step is to rotate the squark states from the Super-CKM basis, $\tilde{q}_{L, R}$, to the physical basis, $\tilde{q}^{\text {phys }}$. If we set the order in the Super-CKM basis as above, $\left(\tilde{u}_{L}, \tilde{c}_{L}, \tilde{t}_{L}, \tilde{u}_{R}, \tilde{c}_{R}, \tilde{t}_{R}\right)$ and $\left(\tilde{d}_{L}, \tilde{s}_{L}, \tilde{b}_{L}, \tilde{d}_{R}, \tilde{s}_{R}, \tilde{b}_{R}\right)$, and in the physical basis as $\tilde{u}_{1, \ldots 6}$ and 
$\tilde{d}_{1, \ldots 6}$, respectively, these last rotations are given by two $6 \times 6$ matrices, $R^{\tilde{u}}$ and $R^{\tilde{d}}$,

$$
\left(\begin{array}{c}
\tilde{u}_{1} \\
\tilde{u}_{2} \\
\tilde{u}_{3} \\
\tilde{u}_{4} \\
\tilde{u}_{5} \\
\tilde{u}_{6}
\end{array}\right)=R^{\tilde{u}}\left(\begin{array}{c}
\tilde{u}_{L} \\
\tilde{c}_{L} \\
\tilde{t}_{L} \\
\tilde{u}_{R} \\
\tilde{c}_{R} \\
\tilde{t}_{R}
\end{array}\right), \quad\left(\begin{array}{c}
\tilde{d}_{1} \\
\tilde{d}_{2} \\
\tilde{d}_{3} \\
\tilde{d}_{4} \\
\tilde{d}_{5} \\
\tilde{d}_{6}
\end{array}\right)=R^{\tilde{d}}\left(\begin{array}{c}
\tilde{d}_{L} \\
\tilde{s}_{L} \\
\tilde{b}_{L} \\
\tilde{d}_{R} \\
\tilde{s}_{R} \\
\tilde{b}_{R}
\end{array}\right),
$$

yielding the diagonal mass-squared matrices as follows,

$$
\begin{array}{r}
\operatorname{diag}\left\{m_{\tilde{u}_{1}}^{2}, m_{\tilde{u}_{2}}^{2}, m_{\tilde{u}_{3}}^{2}, m_{\tilde{u}_{4}}^{2}, m_{\tilde{u}_{5}}^{2}, m_{\tilde{u}_{6}}^{2}\right\}=R^{\tilde{u}} \mathcal{M}_{\tilde{u}}^{2} R^{\tilde{u} \dagger}, \\
\operatorname{diag}\left\{m_{\tilde{d}_{1}}^{2}, m_{\tilde{d}_{2}}^{2}, m_{\tilde{d}_{3}}^{2}, m_{\tilde{d}_{4}}^{2}, m_{\tilde{d}_{5}}^{2}, m_{\tilde{d}_{6}}^{2}\right\}=R^{\tilde{d}} \mathcal{M}_{\tilde{d}}^{2} R^{\tilde{d} \dagger} .
\end{array}
$$

The corresponding Feynman rules in the physical basis for the vertices needed for our applications, i.e. the interaction of one and two Higgs or gauge bosons with two squarks, can be found in the appendix A. This new set of generalized vertices had been implemented into the program packages FeynArts/FormCalc $[50,51,53]^{5}$ extending the previous MSSM model file [54]. The extended FeynArts version was used for the evaluation of the Feynman diagrams along this paper to obtain the general analytical results.

In the numerical part of the present study we will restrict ourselves to the case where there are flavor mixings exclusively between the second and third squark generation. These mixings are known to produce the largest flavor violation effects in $B$ meson physics since their size are usually governed by the third generation quark masses. On the other hand, and in order to reduce further the number of independent parameters, we will focus in the following analysis on constrained SUSY scenarios, where the soft mass parameters fulfill universality hypothesis at the gauge unification (GUT) scale. Concretely, we will work with the so-called Constrained MSSM (CMSSM) and Non Universal Higgs Mass (NUHM) scenarios, which are defined by(see [56] and references therein),

$$
\begin{array}{cc}
\text { CMSSM : } & m_{0}, m_{1 / 2}, A_{0}, \operatorname{sign}(\mu), \tan \beta \\
\text { NUHM : } & m_{0}, m_{1 / 2}, A_{0}, \operatorname{sign}(\mu), \tan \beta, m_{H_{1}}, m_{H_{2}},
\end{array}
$$

where, $A_{0}$ is the universal trilinear coupling, $m_{0}, m_{1 / 2}, m_{H_{1}}, m_{H_{2}}$, are the universal scalar mass, gaugino mass, and $H_{1}$ and $H_{2}$ Higgs masses, respectively, at the GUT scale, $\operatorname{sign}(\mu)$ is the sign of the $\mu$ parameter and again $\tan \beta=v_{2} / v_{1}$. The soft Higgs masses in the NUHM are usually parametrized as $m_{H_{1,2}}^{2}=\left(1+\delta_{1,2}\right) m_{0}^{2}$, such that by taking $\delta_{1,2}=0$ one moves from the NUHM to the CMSSM.

It should be noted that the condition of universal squark soft masses, $m_{\tilde{U}_{L} i j}^{2}=m_{\tilde{U}_{R} i j}^{2}=m_{\tilde{D}_{L} i j}^{2}=m_{\tilde{D}_{R} i j}^{2}=m_{0}^{2} \delta_{i j}$, is fulfilled just at the GUT scale. When running these soft mass matrices from the GUT scale down to the relevant low energy, they will generically turn non-diagonal in flavor. However, in MFV scenarios the non-diagonal terms are exclusively generated in this running by off-diagonal terms in the $V_{\mathrm{CKM}}$, and therefore

\footnotetext{
${ }^{5}$ The program and the user's guide are available via [52].
} 
they can be safely neglected at low energy. Contrary, in NMFV scenarios, the universal hypothesis in these squark mass matrices is by definition not fulfilled at low energies.

Our final settings for the numerical evaluation of the squark flavor mixings in NMFV scenarios are fixed (after RGE running) at low energy as follows,

$$
\begin{aligned}
& m_{\tilde{U}_{L}}^{2}=\left(\begin{array}{ccc}
m_{\tilde{U}_{L 11}}^{2} & 0 & 0 \\
0 & m_{\tilde{U}_{L 22}}^{2} & \delta_{23}^{L L} m_{\tilde{U}_{L 22}} m_{\tilde{U}_{L 33}} \\
0 & \delta_{23}^{L L} m_{\tilde{U}_{L 22}} m_{\tilde{U}_{L 33}} & m_{\tilde{U}_{L 33}}^{2}
\end{array}\right) \\
& v_{2} \mathcal{A}^{u}=\left(\begin{array}{ccc}
0 & 0 & 0 \\
0 & 0 & \delta_{c t}^{L R} m_{\tilde{U}_{L 22}} m_{\tilde{U}_{R 33}} \\
0 & \delta_{c t}^{R L} m_{\tilde{U}_{R 22}} m_{\tilde{U}_{L 33}} & m_{t} A_{t}
\end{array}\right) \\
& m_{\tilde{U}_{R}}^{2}=\left(\begin{array}{ccc}
m_{\tilde{U}_{R 11}}^{2} & 0 & 0 \\
0 & m_{\tilde{U}_{R 22}}^{2} & \delta_{c t}^{R R} m_{\tilde{U}_{R 22}} m_{\tilde{U}_{R 33}} \\
0 & \delta_{c t}^{R R} m_{\tilde{U}_{R 22}} m_{\tilde{U}_{R 33}} & m_{\tilde{U}_{R 33}}^{2}
\end{array}\right) \\
& m_{\tilde{D}_{L}}^{2}=V_{\mathrm{CKM}}^{\dagger} m_{\tilde{U}_{L}}^{2} V_{\mathrm{CKM}} \\
& v_{1} \mathcal{A}^{d}=\left(\begin{array}{lcc}
0 & 0 & 0 \\
0 & 0 & \delta_{s b}^{L R} m_{\tilde{D}_{L 22}} m_{\tilde{D}_{R 33}} \\
0 \delta_{s b}^{R L} m_{\tilde{D}_{R 22}} m_{\tilde{D}_{L 33}} & m_{b} A_{b}
\end{array}\right) \\
& m_{\tilde{D}_{R}}^{2}=\left(\begin{array}{ccc}
m_{\tilde{D}_{R 11}}^{2} & 0 & 0 \\
0 & m_{\tilde{D}_{R 22}}^{2} & \delta_{s b}^{R R} m_{\tilde{D}_{R 22}} m_{\tilde{D}_{R 33}} \\
0 & \delta_{s b}^{R R} m_{\tilde{D}_{R 22}} m_{\tilde{D}_{R 33}} & m_{\tilde{D}_{R 33}}^{2}
\end{array}\right)
\end{aligned}
$$

It is worth mentioning that the relation between the two soft squark mass matrices in the 'Left' sector (2.21) is due to $\mathrm{SU}(2)$ gauge invariance. Eq. (2.21) can be derived from the two last relations of eq. (2.11). This dependence between the non-diagonal terms of these squark mass matrices is the reason why is introduced $\delta_{23}^{L L}$ instead of two independent deltas $\delta_{c t}^{L L}$ and $\delta_{s b}^{L L}$. To get the needed running of the soft parameters from the GUT scale down to low energy, that we set here $1 \mathrm{TeV}$, we solve numerically the one-loop RGEs with the code SPHENO [57]. The diagonalization of all the mass matrices is performed with the program FeynHiggs [23, 24, 29-31].

In CMSSM and other SUSY-GUT scenarios the flavor changing deltas go (in the leading logarithmic approximation) as $\delta_{23}^{L L} \simeq-\frac{1}{8 \pi^{2}} \frac{\left(3 m_{0}^{2}+A_{0}^{2}\right)}{\tilde{m}^{2}}\left(Y^{{ }^{\dagger}} Y^{q}\right)_{23} \log \left(\frac{M_{\mathrm{GUT}}}{M_{\mathrm{EW}}}\right)\left(\tilde{m}^{2}\right.$ is usually taken as the geometric mean of the involved flavor diagonal squared squark mass matrix entries, see eq. (2.24)), whereas the $L R, R L$ and $R R$ deltas are suppressed instead by small mass ratios, $\sim \frac{\left(m_{q} A_{0}\right)}{\tilde{m}^{2}}$ and $\sim \frac{\left(m_{q}^{2}\right)}{\tilde{m}^{2}}$, respectively. Furthermore, in these scenarios the mixing involving the first generation squarks is naturally suppressed by the smallness of the corresponding Yukawa couplings. In order to keep the number of free parameters manageable, this motivated our above choice of neglecting in the numerical analysis the mixing of the first generation squarks. However, we will not assume any explicit hierarchy in the various mixing terms between the second and third generation. 
It should be noted that in the 'Left-Right' sector we have normalized the trilinear couplings at low energies as $\mathcal{A}_{i j}^{q}=y_{q_{i}} A_{i j}^{q}$ (with $A_{33}^{u}=A_{t}$ and $A_{33}^{d}=A_{b}$ ) and we have neglected the $A_{i}$ couplings of the first and second generations. Finally, it should be noted that the dimensionless parameters $\delta_{i j}^{X Y}$ defining the non-diagonal entries in flavor space $(i \neq j)$ are normalized respect the geometric mean of the corresponding diagonal squared soft masses. For instance,

$$
\begin{array}{ll}
\delta_{23}^{L L}=m_{\tilde{U}_{L 23}}^{2} /\left(m_{\tilde{U}_{L 22}} m_{\tilde{U}_{L 33}}\right), & \delta_{c t}^{R R}=m_{\tilde{U}_{R 23}}^{2} /\left(m_{\tilde{U}_{R 22}} m_{\tilde{U}_{R 33}}\right), \\
\delta_{c t}^{L R}=\left(v_{2} \mathcal{A}^{u}\right)_{23} /\left(m_{\tilde{U}_{L 22}} m_{\tilde{U}_{R 33}}\right), & \delta_{c t}^{R L}=\left(v_{2} \mathcal{A}^{u}\right)_{32} /\left(m_{\tilde{U}_{R 22}} m_{\tilde{U}_{L 33}}\right), \quad \text { etc. }
\end{array}
$$

For definiteness and simplicity, in the present work we will perform our estimates in specific constrained SUSY scenarios, CMSSM and NUHM, whose input parameters $m_{0}$, $m_{1 / 2}, A_{0}, \tan \beta, \operatorname{sign}(\mu), \delta_{1,2}$, are summarized in table $1,{ }^{6}$ and supplemented with $\delta_{i j}^{X Y}$ as described above. Regarding CMSSM, we have chosen six SPS benchmark points [58], SPS1a, SPS1b, SPS2, SPS3, SPS4, and SPS5 and one more point with very heavy spectra (VHeavyS). It should be noted that several of these SPSX points are already in conflict with recent LHC data [6, 7], but we have chosen them here as reference points to study the effects of SUSY on the Higgs mass corrections, since they have been studied at length in the literature. At present, a heavier SUSY spectrum, as for instance our point VHeavyS is certainly more realistic and compatible with LHC data. In general an analysis of LHC data including NMFV effects in the squark sector would be very desirable. Regarding NUHM, we have chosen a point with heavy SUSY spectra and light Higgs sector (HeavySLightH) and a point (BFP) that has been proven in [59] to give the best fit to the set of low energy observables. For later reference, needed in our posterior analysis of the Higgs mass corrections, we also include in the table the corresponding MSSM Higgs masses, computed with FeynHiggs $[23,24,29-31]$ and with all flavor changing deltas switched off, i.e., $\delta_{i j}^{X Y}=0$.

\section{Constraints on Non-Minimal Flavor Violating SUSY scenarios from $B$-physics}

In this section we analyze the constraints on Non-Minimal Flavor Violating SUSY scenarios from $B$-Physics. Since we are mainly interested in the phenomenological consequences of the flavor mixing between the third and second generations we will focus ${ }^{7}$ on the following three $\mathrm{B}$ meson observables: 1$)$ Branching ratio of the $B$ radiative decay $\left.\mathrm{BR}\left(B \rightarrow X_{s} \gamma\right), 2\right)$ Branching ratio of the $B_{s}$ muonic decay $\mathrm{BR}\left(B_{s} \rightarrow \mu^{+} \mu^{-}\right)$, and 3) $B_{s}-\bar{B}_{s}$ mass difference $\Delta M_{B_{s}}$. Another $B$ observable of interest in the present context is $\operatorname{BR}\left(B \rightarrow X_{s} l^{+} l^{-}\right)$. However, we have not included this in our study, because the predicted rates in NMFVSUSY scenarios for this observable are closely correlated with those from $\operatorname{BR}\left(B \rightarrow X_{s} \gamma\right)$ due to the dipole operators dominance in the photon-penguin diagrams mediating $\mathrm{BR}(B \rightarrow$

\footnotetext{
${ }^{6}$ We adopt here the definition in terms of the GUT-scale input parameters, while the original definition in [58] was based on the weak-scale parameters.

${ }^{7}$ We have checked that electroweak precision observables, where NMFV effects enter, for instance, via $\Delta \rho[28]$, do not lead to relevant additional constraints on the allowed parameter space. Our results on this constraint are in agreement with ref. [37].
} 


\begin{tabular}{|c|c|c|c|c|c|c|c|c|c|c|}
\hline points & $m_{1 / 2}$ & $m_{0}$ & $A_{0}$ & $\tan \beta$ & $\delta_{1}$ & $\delta_{2}$ & $m_{h}$ & $m_{H}$ & $M_{A}$ & $m_{H^{ \pm}}$ \\
\hline SPS1 a & 250 & 100 & -100 & 10 & 0 & 0 & 112 & 394 & 394 & 402 \\
SPS1 b & 400 & 200 & 0 & 30 & 0 & 0 & 116 & 526 & 526 & 532 \\
SPS2 & 300 & 1450 & 0 & 10 & 0 & 0 & 115 & 1443 & 1443 & 1445 \\
SPS3 & 400 & 90 & 0 & 10 & 0 & 0 & 115 & 573 & 572 & 578 \\
SPS4 & 300 & 400 & 0 & 50 & 0 & 0 & 114 & 404 & 404 & 414 \\
SPS5 & 300 & 150 & -1000 & 5 & 0 & 0 & 111 & 694 & 694 & 698 \\
VHeavyS & 800 & 800 & -800 & 5 & 0 & 0 & 120 & 1524 & 1524 & 1526 \\
HeavySLightH & 600 & 600 & 0 & 5 & -1.86 & +1.86 & 114 & 223 & 219 & 233 \\
BFP & 530 & 110 & -370 & 27 & -84.7 & -84.7 & 120 & 507 & 507 & 514 \\
\hline
\end{tabular}

Table 1. Values of $m_{1 / 2}, m_{0}, A_{0}, \tan \beta, \delta_{1}, \delta_{2}$ and Higgs boson masses $m_{h}, m_{H}, M_{A}$ and $m_{H^{ \pm}}$ for the points considered in the analysis. All parameters with mass dimension are in $\mathrm{GeV}$, and $\operatorname{sign}(\mu)>0$ for all points.

$\left.X_{s} l^{+} l^{-}\right)$decays. It implies that the restrictions on the flavor mixing $\delta_{i j}^{X Y}$ parameters from $\mathrm{BR}\left(B \rightarrow X_{s} l^{+} l^{-}\right)$are also expected to be correlated with those from the radiative decays.

The summary of the relevant features for our analysis of these three B meson observables is given in the following.

\section{$3.1 \quad \operatorname{BR}\left(B \rightarrow X_{s} \gamma\right)$}

The relevant effective Hamiltonian for this decay is given in terms of the Wilson coefficients $C_{i}$ and operators $O_{i}$ by:

$$
\mathcal{H}_{\text {eff }}=-\frac{4 G_{F}}{\sqrt{2}} V_{\text {CKM }}^{t s *} V_{\text {CKM }}^{t b} \sum_{i=1}^{8}\left(C_{i} O_{i}+C_{i}^{\prime} O_{i}^{\prime}\right) .
$$

Where the primed operators can be obtained from the unprimed ones by replacing $L \leftrightarrow R$. The complete list of operators can be found, for instance, in [55]. In the context of SUSY scenarios with the MSSM particle content and MFV, only two of these operators get relevant contributions, the so-called photonic dipole operator $O_{7}$ and gluonic dipole operator $O_{8}$ given, respectively, by:

$$
\begin{aligned}
& O_{7}=\frac{e}{16 \pi^{2}} m_{b}\left(\bar{s}_{L} \sigma^{\mu \nu} b_{R}\right) F_{\mu \nu}, \\
& O_{8}=\frac{g_{3}}{16 \pi^{2}} m_{b}\left(\bar{s}_{L} \sigma^{\mu \nu} T^{a} b_{R}\right) G_{\mu \nu}^{a} .
\end{aligned}
$$

We have omitted the color indices here for brevity. Within NMFV also $O_{7,8}^{\prime}$ have to be taken into account:

$$
\begin{aligned}
& O_{7}^{\prime}=\frac{e}{16 \pi^{2}} m_{b}\left(\bar{s}_{R} \sigma^{\mu \nu} b_{L}\right) F_{\mu \nu} \\
& O_{8}^{\prime}=\frac{g_{3}}{16 \pi^{2}} m_{b}\left(\bar{s}_{R} \sigma^{\mu \nu} T^{a} b_{L}\right) G_{\mu \nu}^{a} .
\end{aligned}
$$


The Wilson coefficients at the SUSY scale are obtained as usual by the matching procedure of the proper matrix element being computed from the previous effective Hamiltonian to the corresponding matrix elements being computed from the SUSY model operating at that SUSY scale, the NMFV-MSSM in our case. These Wilson coefficients encode, therefore, the contributions to $\mathrm{BR}\left(B \rightarrow X_{s} \gamma\right)$ from the loops of the SUSY and Higgs sectors of the MSSM. The effects from squark flavor mixings that are parametrized by the $\delta_{i j}^{X Y}$, are included in this observable via the squark physical masses and rotation matrices, given in the previous section, that appear in the computation of the matrix element at the one loop level and, therefore, are also encoded in the Wilson coefficients. The explicit expressions for these coefficients in the MSSM, in terms of the physical basis, can be found, for instance, in refs. [60-62]. We have included in our analysis the most relevant loop contributions to the Wilson coefficients, concretely: 1) loops with Higgs bosons, 2) loops with charginos and 3) loops with gluinos. It should be noted that, at one loop order, the gluino loops do not contribute in MFV scenarios, but they are very relevant (dominant in many cases) in the present NMFV scenarios.

Once the Wilson coefficients are known at the SUSY scale, they are evolved with the proper Renormalization Group Equations (RGEs) down to the proper low-energy scale. As a consequence of this running the previous operators mix and the corresponding Wilson coefficients, $C_{7,8}$ get involved in the computation of the $B \rightarrow X_{s} \gamma$ decay rate. The RGE-running is done in two steps: The first one is from the SUSY scale down to the electroweak scale, and the second one is from this electroweak scale down to the B-physics scale. For the first step, we use the LO-RGEs for the relevant Wilson coefficients as in [62] and fix six active quark flavors in this running. For the second running we use the NLO-RGEs as in [63] and fix, correspondingly, five active quark flavors. For the charged Higgs sector we use the NLO formulas for the Wilson coefficients as in [64].

The resummation of scalar induced large $\tan \beta$ effects is performed, as usual, by the effective Lagrangian approach that parametrizes the one-loop generated effective couplings between the $\mathcal{H}_{2}$ Higgs doublet and the down type quarks in softly broken SUSY models [65]. A necessary condition to take into account all $\tan \beta$-enhanced terms in flavor changing amplitudes is the diagonalization of the down-type quark mass matrix in the presence of these effective couplings [66-68]. A summary of this effective Lagrangian formalism for the resummation of large $\tan \beta$ effects in the three $B$ observables of our interest, within the context of MFV scenarios, can be found in [69]. We follow here the treatment of [70] where the resummation of large $\tan \beta$ effects via effective Lagrangians is generalized to the case where the effective $\bar{d}_{R}^{i} d_{L}^{j} \mathcal{H}_{2}^{0}$ coupling contains also non-minimal sources of flavor mixing. It should be noted that the most relevant scalar induced large $\tan \beta$ effects for the present work are those generated by one-loop diagrams with gluino-sbottom and chargino-stop inside the loops. The large $\tan \beta$ resummation effects and the relevance of other chirally enhanced corrections to FCNC processes within the NMFV context have recently been studied exhaustively also in $[71,72]$ (previous studies can be found, for instance, in refs. [73-75]).

The total branching ratio for this decay is finally estimated by adding the new contributions from the SUSY and Higgs sectors to the SM rate. More specifically, we use eq. (42) of [63] for the estimate of $\operatorname{BR}\left(B \rightarrow X_{s} \gamma\right)$ in terms of the ratios of the Wilson 
coefficients $C_{7,8}$ and $C_{7,8}^{\prime}$ (including all the mentioned new contributions) divided by the corresponding $C_{7,8}^{\mathrm{SM}}$ in the $\mathrm{SM}$.

For the numerical estimates of $\operatorname{BR}\left(B \rightarrow X_{s} \gamma\right)$ we use the FORTRAN subroutine BPHYSICS (modified as to include the contributions from $C_{7,8}^{\prime}$ which were not included in its original version) included in the SuFla code, that incorporates all the above mentioned ingredients $[42,43]$.

Finally, for completeness, we include below the experimental measurement of this observable $[8,76],{ }^{8}$ and its prediction within the SM [77]:

$$
\begin{aligned}
& \mathrm{BR}\left(B \rightarrow X_{s} \gamma\right)_{\exp }=(3.55 \pm 0.26) \times 10^{-4} \\
& \mathrm{BR}\left(B \rightarrow X_{s} \gamma\right)_{\mathrm{SM}}=(3.15 \pm 0.23) \times 10^{-4}
\end{aligned}
$$

\section{$3.2 \operatorname{BR}\left(B_{s} \rightarrow \mu^{+} \mu^{-}\right)$}

The relevant effective Hamiltonian for this process is [78, 79]:

$$
\mathcal{H}_{\mathrm{eff}}=-\frac{G_{F} \alpha}{\sqrt{2} \pi} V_{\mathrm{CKM}}^{t s *} V_{\mathrm{CKM}}^{t b} \sum_{i}\left(C_{i} O_{i}+C_{i}^{\prime} O_{i}^{\prime}\right),
$$

where the operators $O_{i}$ are given by:

$$
\begin{aligned}
O_{10} & =\left(\bar{s} \gamma^{\nu} P_{L} b\right)\left(\bar{\mu} \gamma_{\nu} \gamma_{5} \mu\right), & O_{10}^{\prime} & =\left(\bar{s} \gamma^{\nu} P_{R} b\right)\left(\bar{\mu} \gamma_{\nu} \gamma_{5} \mu\right), \\
O_{S} & =m_{b}\left(\bar{s} P_{R} b\right)(\bar{\mu} \mu), & O_{S}^{\prime} & =m_{s}\left(\bar{s} P_{L} b\right)(\bar{\mu} \mu), \\
O_{P} & =m_{b}\left(\bar{s} P_{R} b\right)\left(\bar{\mu} \gamma_{5} \mu\right), & O_{P}^{\prime} & =m_{s}\left(\bar{s} P_{L} b\right)\left(\bar{\mu} \gamma_{5} \mu\right) .
\end{aligned}
$$

We have again omitted the color indices here for brevity.

In this case, the RG running is straightforward since the anomalous dimensions of the above involved operators are zero, and the prediction for the decay rate is simply expressed by:

$$
\begin{aligned}
\operatorname{BR}\left(B_{s} \rightarrow \mu^{+} \mu^{-}\right)= & \frac{G_{F}^{2} \alpha^{2} m_{B_{s}}^{2} f_{B_{s}}^{2} \tau_{B_{s}}}{64 \pi^{3}}\left|V_{\mathrm{CKM}}^{t s *} V_{\mathrm{CKM}}^{t b}\right|^{2} \sqrt{1-4 \hat{m}_{\mu}^{2}} \\
& \times\left[\left(1-4 \hat{m}_{\mu}^{2}\right)\left|F_{S}\right|^{2}+\left|F_{P}+2 \hat{m}_{\mu}^{2} F_{10}\right|^{2}\right],
\end{aligned}
$$

where $\hat{m}_{\mu}=m_{\mu} / m_{B_{s}}$ and the $F_{i}$ are given by

$$
F_{S, P}=m_{B_{s}}\left[\frac{C_{S, P} m_{b}-C_{S, P}^{\prime} m_{s}}{m_{b}+m_{s}}\right], \quad \quad F_{10}=C_{10}-C_{10}^{\prime} .
$$

Within the SM the most relevant operator is $O_{10}$ as the Higgs mediated contributions to $O_{S, P}$ can be safely neglected. It should be noted that the contribution from $O_{10}$ to the decay rate is helicity suppressed by a factor of $\hat{m}_{\mu}^{2}$ since the $B_{s}$ meson has spin zero. In contrast, in SUSY scenarios the scalar and pseudo-scalar operators, $O_{S, P}$, can be very important, particularly at large $\tan \beta \gtrsim 30$ where the contributions to $C_{S}$ and $C_{P}$ from neutral Higgs penguin diagrams can become large and dominate the branching ratio,

\footnotetext{
${ }^{8}$ We have added the various contributions to the experimental error in quadrature.
} 
because in this case the branching ratio grows with $\tan \beta$ as $\tan ^{6} \beta$. The studies in the literature of these MSSM Higgs-penguin contributions to $\operatorname{BR}\left(B_{s} \rightarrow \mu^{+} \mu^{-}\right)$have focused on both MFV $[68,80,81]$ and NMFV scenarios [45, 70, 75, 78]. In both cases the rates for $\mathrm{BR}\left(B_{s} \rightarrow \mu^{+} \mu^{-}\right)$at large $\tan \beta$ can be enhanced by a few orders of magnitude compared with the prediction in the SM, therefore providing an optimal window for SUSY signals.

In the present context of SUSY-NMFV, with no preference for large $\tan \beta$ values, there are in general three types of one-loop diagrams that contribute to the previous $C_{i}$ Wilson coefficients for this $B_{s} \rightarrow \mu^{+} \mu^{-}$decay: 1) Box diagrams, 2) $Z$-penguin diagrams and 3) neutral Higgs boson $H$-penguin diagrams, where $H$ denotes the three neutral MSSM Higgs bosons. In our numerical estimates we have included what are known to be the dominant contributions to these three types of diagrams [78]: chargino contributions to box and Z-penguin diagrams and chargino and gluino contributions to $H$-penguin diagrams.

Regarding the resummation of large $\tan \beta$ effects we have followed the same effective Lagrangian formalism as explained in the previous case of $B \rightarrow X_{s} \gamma$. In the case of contributions from $H$-penguin diagrams to $B_{s} \rightarrow \mu^{+} \mu^{-}$this resummation is very relevant and it has been generalized to NMFV-SUSY scenarios in [70].

For the numerical estimates we use again the BPHYSICS subroutine included in the SuFla code $[42,43]$ which incorporates all the ingredients that we have pointed out above.

Finally, for completeness, we include below the present experimental upper bound for this observable [82], and the prediction within the SM [83]:

$$
\begin{aligned}
& \operatorname{BR}\left(B_{s} \rightarrow \mu^{+} \mu^{-}\right)_{\exp }<1.1 \times 10^{-8} \quad(95 \% \mathrm{CL}) \\
& \operatorname{BR}\left(B_{s} \rightarrow \mu^{+} \mu^{-}\right)_{\mathrm{SM}}=(3.6 \pm 0.4) \times 10^{-9}
\end{aligned}
$$

\section{$3.3 \Delta M_{B_{s}}$}

The relevant effective Hamiltonian for $B_{s}-\bar{B}_{s}$ mixing and, hence, for the $B_{s} / \bar{B}_{s}$ mass difference $\Delta M_{B_{s}}$ is:

$$
\mathcal{H}_{\mathrm{eff}}=\frac{G_{F}^{2}}{16 \pi^{2}} M_{W}^{2}\left(V_{\mathrm{CKM}}^{t b *} V_{\mathrm{CKM}}^{t s}\right)^{2} \sum_{i} C_{i} O_{i} .
$$

In the SM the most relevant operator is:

$$
O^{V L L}=\left(\bar{b}^{\alpha} \gamma_{\mu} P_{L} s^{\alpha}\right)\left(\bar{b}^{\beta} \gamma^{\mu} P_{L} s^{\beta}\right) .
$$

Where we have now written explicitly the color indices. In scenarios beyond the SM, as the present NMFV MSSM, other operators are also relevant:

$$
\begin{aligned}
O_{1}^{L R} & =\left(\bar{b}^{\alpha} \gamma_{\mu} P_{L} s^{\alpha}\right)\left(\bar{b}^{\beta} \gamma^{\mu} P_{R} s^{\beta}\right), & O_{2}^{L R} & =\left(\bar{b}^{\alpha} P_{L} s^{\alpha}\right)\left(\bar{b}^{\beta} P_{R} s^{\beta}\right), \\
O_{1}^{S L L} & =\left(\bar{b}^{\alpha} P_{L} s^{\alpha}\right)\left(\bar{b}^{\beta} P_{L} s^{\beta}\right), & O_{2}^{S L L} & =\left(\bar{b}^{\alpha} \sigma_{\mu \nu} P_{L} s^{\alpha}\right)\left(\bar{b}^{\beta} \sigma^{\mu \nu} P_{L} s^{\beta}\right),
\end{aligned}
$$

and the corresponding operators $O^{V R R}$ and $O_{i}^{S R R}$ that can be obtained by replacing $P_{L} \leftrightarrow P_{R}$ in (3.14) and (3.16). The mass difference $\Delta M_{B_{s}}$ is then evaluated by taking the matrix element

$$
\Delta M_{B_{s}}=2\left|\left\langle\bar{B}_{s}\left|\mathcal{H}_{\mathrm{eff}}\right| B_{s}\right\rangle\right|
$$


where $\left\langle\bar{B}_{s}\left|\mathcal{H}_{\text {eff }}\right| B_{s}\right\rangle$ is given by

$$
\left\langle\bar{B}_{s}\left|\mathcal{H}_{\mathrm{eff}}\right| B_{s}\right\rangle=\frac{G_{F}^{2}}{48 \pi^{2}} M_{W}^{2} m_{B_{s}} f_{B_{s}}^{2}\left(V_{\mathrm{CKM}}^{t b *} V_{\mathrm{CKM}}^{t s}\right)^{2} \sum_{i} P_{i} C_{i}\left(\mu_{W}\right) .
$$

Here $m_{B_{s}}$ is the $B_{s}$ meson mass, and $f_{B_{s}}$ is the $B_{s}$ decay constant. The coefficients $P_{i}$ contain the effects due to RG running between the electroweak scale $\mu_{W}$ and $m_{b}$ as well as the relevant hadronic matrix element. We use the coefficients $P_{i}$ from the lattice calculation $[84,85]$ :

$$
P_{1}^{V L L}=0.73, \quad P_{1}^{L R}=-1.97, \quad P_{2}^{L R}=2.50, \quad P_{1}^{S L L}=-1.02, \quad P_{2}^{S L L}=-1.97 .
$$

The coefficients $P_{1}^{V R R}$, etc., may be obtained from those above by simply exchanging $L \leftrightarrow R$.

In the present context of SUSY-NMFV, again with no preference for large $\tan \beta$ values, and besides the SM loop contributions, there are in general three types of one-loop diagrams that contribute to the previous $C_{i}$ Wilson coefficients for $B_{s}-\bar{B}_{s}$ mixing: 1) Box diagrams, 2) $Z$-penguin diagrams and 3) double Higgs-penguin diagrams. In our numerical estimates we have included what are known to be the dominant contributions to these three types of diagrams in scenarios with non-minimal flavor violation (for a review see, for instance, [45]): gluino contributions to box diagrams, chargino contributions to box and Z-penguin diagrams, and chargino and gluino contributions to double $H$-penguin diagrams. As in the previous observables, the total prediction for $\Delta M_{B_{s}}$ includes, of course, the SM contribution.

Regarding the resummation of large $\tan \beta$ effects we have followed again the effective Lagrangian formalism, generalized to NMFV-SUSY scenarios [70], as in the previous cases of $B \rightarrow X_{s} \gamma$ and $B_{s} \rightarrow \mu^{+} \mu^{-}$. It should be noted that, in the case of $\Delta M_{B_{s}}$, the resummation of large $\tan \beta$ effects is very relevant for the double $H$-penguin contributions, which grow very fast with $\tan \beta$.

For the numerical estimates we have modified the BPHYSICS subroutine included in the SuFla code $[42,43]$ which incorporates all the ingredients that we have pointed out above, except the contributions from gluino boxes. These contributions are known to be very important for $B_{s}-\bar{B}_{s}$ mixing in SUSY scenarios with non-minimal flavor violation [4547] and therefore they must be included into our analysis of $\Delta M_{B_{s}}$. Concretely, we have incorporated them into the BPHYSICS subroutine by adding the full one-loop formulas for the gluino boxes of [44] to the other above quoted contributions that were already included in the code. In order to illustrate the relevance of these gluino contributions in our analysis of $\Delta M_{B_{s}}$, we show in figure 1 each separate contribution as a function of $\tan \beta$ in a particular example with $\delta_{23}^{L L}=\delta_{s b}^{R R}=0.1$, that we have chosen for comparison with [45]. The other flavor changing deltas are set to zero, and the other relevant MSSM parameters are set to $m_{\tilde{q}}=$ $500 \mathrm{GeV}, A_{t}=-m_{\tilde{q}}, m_{\tilde{g}}=\sqrt{2} m_{\tilde{q}}, \mu=m_{\tilde{q}}$, and $m_{A}=300 \mathrm{GeV}$, as in figure 24 of [45]. We clearly see in figure 1 that it is just in the very large $\tan \beta$ region where double Higgspenguins dominate. For moderate and low $\tan \beta$ values, $\tan \beta \leq 20$ (which is a relevant region for our numerical analysis, see below) the gluino boxes fully dominates the SUSY corrections to $\Delta M_{B_{s}}$ and compete with the SM contributions. Our numerical estimate in 


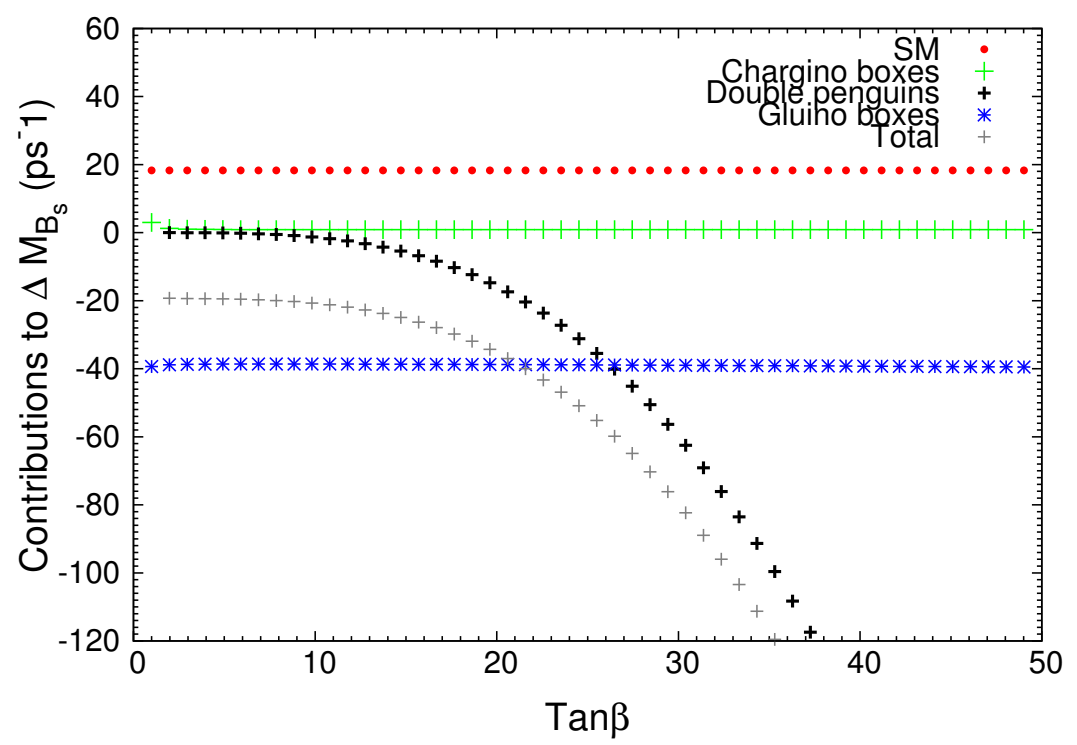

Figure 1. Relevant contributions to $\Delta M_{B_{s}}$ in NMFV-SUSY scenarios as a function of $\tan \beta$. They include: SM, Double Higgs penguins, gluino boxes and chargino boxes. The total prediction for $\Delta M_{B_{s}}$ should be understood here as $\Delta M_{B_{s}}=\mid$ Total|. The parameters are set to $\delta_{23}^{L L}=\delta_{s b}^{R R}=0.1, m_{\tilde{q}}=500 \mathrm{GeV}, A_{t}=-m_{\tilde{q}}, m_{\tilde{g}}=\sqrt{2} m_{\tilde{q}}, \mu=m_{\tilde{q}}$, and $m_{A}=300 \mathrm{GeV}$. The other flavor changing deltas are set to zero.

this plot is in complete agreement with the results in [45] (see, in particular, figure 24 of this reference) which analyzed and compared in full detail these corrections. Finally, for completeness, we include below the experimental measurement of this observable $[8],{ }^{9}$ and its prediction within the SM (using NLO expression of [86] and error estimate of [87]):

$$
\begin{aligned}
& \Delta M_{B_{s} \exp }=(117.0 \pm 0.8) \times 10^{-10} \mathrm{MeV} \\
& \Delta M_{B_{s} \mathrm{SM}}=\left(117.1_{-16.4}^{+17.2}\right) \times 10^{-10} \mathrm{MeV} .
\end{aligned}
$$

\subsection{Numerical results on $B$ observables}

In the following of this section we present our numerical results for the three $B$ observables in the NMFV-SUSY scenarios and a discussion on the allowed values for the flavor changing deltas, $\delta_{i j}^{X Y}$.

The predictions for $\operatorname{BR}\left(B \rightarrow X_{s} \gamma\right), \operatorname{BR}\left(B_{s} \rightarrow \mu^{+} \mu^{-}\right)$and $\Delta M_{B_{s}}$ versus the various $\delta_{i j}^{X Y}$, for the six selected SPS points, are displayed respectively in figures 2,3 and 4 . For this analysis, we have assumed that just one at a time of these deltas is not vanishing. Results for two non-vanishing deltas will be shown later. The following 7 flavor changing deltas are considered: $\delta_{23}^{L L}, \delta_{c t}^{L R}, \delta_{s b}^{L R}, \delta_{c t}^{R L}, \delta_{s b}^{R L}, \delta_{c t}^{R R}$ and $\delta_{s b}^{R R}$; and we have explored delta values within the interval $-1<\delta_{i j}^{X Y}<1$. In all plots, the predictions for $\delta_{i j}^{X Y}=0$ represent our estimate of the corresponding observable in the MFV case. This will allow us to compare easily the

\footnotetext{
${ }^{9}$ We have again added the various contributions to the experimental error in quadrature.
} 
results in the two kind of scenarios, NMFV and MFV. It should be noted also, that some of the predicted lines in these plots do not expand along the full interval $-1<\delta_{i j}^{X Y}<1$, and they are restricted to a smaller interval; for some regions of the parameter space a too large delta value can generate very large corrections to any of the masses, and the mass squared turns negative. These problematic points are consequently not shown in our plots.

We have also included in the right vertical axis of these figures, for comparison, the respective SM prediction in (3.7), (3.12), and (3.21). The error bars displayed are the corresponding SM uncertainties as explained below. The shadowed horizontal bands in the case of $\mathrm{BR}\left(B \rightarrow X_{s} \gamma\right)$ and $\Delta M_{B_{s}}$ are their corresponding experimental measurements in (3.6), and (3.20), expanded with $3 \sigma_{\text {exp }}$ errors. In the case of $\operatorname{BR}\left(B_{s} \rightarrow \mu^{+} \mu^{-}\right)$the shadowed area corresponds to the allowed region by the upper bound in (3.11).

The main conclusions extracted from these figures for the three $B$ observables are summarized as follows:

- $\operatorname{BR}\left(B \rightarrow X_{s} \gamma\right)$ :

- Sensitivity to the various deltas:

We find strong sensitivity to $\delta_{s b}^{L R}, \delta_{s b}^{R L}, \delta_{23}^{L L}, \delta_{s b}^{R R}$ and $\delta_{c t}^{L R}$, in all the studied points, for both high and low $\tan \beta$ values. The order found from the highest to the lowest sensitivity is, generically: 1) $\delta_{s b}^{L R}$ and $\delta_{s b}^{R L}$ the largest, 2) $\delta_{23}^{L L}$ the next, 3) $\delta_{c t}^{L R}$ and $\delta_{s b}^{R R}$ the next to next, and 4) slight sensitivity to $\delta_{c t}^{R R}$ and $\delta_{c t}^{R L}$.

- Comparing the predictions with the experimental data:

If we focus on the five most relevant deltas, $\delta_{s b}^{L R}, \delta_{s b}^{R L}, \delta_{23}^{L L}, \delta_{s b}^{R R}$ and $\delta_{c t}^{L R}$, we see clearly that tiny deviations from zero (i.e., deviations from MFV) in these deltas, and specially in the first three, produce sizeable shifts in $\operatorname{BR}\left(B \rightarrow X_{s} \gamma\right)$, and in many cases take it out the experimental allowed band. Therefore, it is obvious from these plots that $\mathrm{BR}\left(B \rightarrow X_{s} \gamma\right)$ sets stringent bounds on the deltas (when varying just one delta), which are particularly tight on $\delta_{s b}^{L R}, \delta_{s b}^{R L}, \delta_{23}^{L L}$, $\delta_{s b}^{R R}$, and $\delta_{c t}^{L R}$, indeed for all the studied SPS points. There are just two exceptions, where the predicted central values of $\operatorname{BR}\left(B \rightarrow X_{s} \gamma\right)$ are already outside the experimental band in the MFV case (all deltas set to zero), and assuming one of these three most relevant deltas to be non-vanishing, the prediction moves inside the experimental band. This happens, for instance, in the points SPS4 and SPS1b that have the largest $\tan \beta$ values of 50 and 30 respectively. Interestingly, it means that some points of the CMSSM, particularly those with large $\tan \beta$ values, that would have been excluded in the context of MFV, can be recovered as compatible with data within NMFV-SUSY scenarios.

- Intervals of $\delta_{i j}^{X Y}$ allowed by data:

In order to conclude on the allowed delta intervals we have assumed that our predictions of $\mathrm{BR}\left(B \rightarrow X_{s} \gamma\right)$ within SUSY scenarios have a somewhat larger theoretical error $\Delta^{\text {theo }}\left(\mathrm{BR}\left(B \rightarrow X_{s} \gamma\right)\right)$ than the SM prediction $\Delta_{\mathrm{SM}}^{\text {theo }}\left(\mathrm{BR}\left(B \rightarrow X_{s} \gamma\right)\right)$ given in (3.7). As a very conservative value we use $\Delta^{\text {theo }}\left(\operatorname{BR}\left(B \rightarrow X_{s} \gamma\right)\right)=0.69 \times 10^{-4}$. A given $\delta_{i j}^{X Y}$ value is then 
considered to be allowed by data if the corresponding interval, defined by $\mathrm{BR}\left(B \rightarrow X_{s} \gamma\right) \pm \Delta^{\text {theo }}\left(\mathrm{BR}\left(B \rightarrow X_{s} \gamma\right)\right)$, intersects with the experimental band. It corresponds to adding linearly the experimental uncertainty and the MSSM theoretical uncertainty. In total a predicted ratio in the interval

$$
2.08 \times 10^{-4}<\operatorname{BR}\left(B \rightarrow X_{s} \gamma\right)<5.02 \times 10^{-4},
$$

is regarded as allowed. Our results for these allowed intervals are summarized in table 2. In this table we see again that the less constrained parameters by $\operatorname{BR}\left(B \rightarrow X_{s} \gamma\right)$ are $\delta_{c t}^{R L}$, and $\delta_{c t}^{R R}$. Therefore, except for the excluded SPS4 case, these two deltas can be sizeable, $\left|\delta_{i j}^{X Y}\right|$ larger than $\mathcal{O}(0.1)$, and compatible with $\operatorname{BR}\left(B \rightarrow X_{s} \gamma\right)$ data.

- $\operatorname{BR}\left(B_{s} \rightarrow \mu^{+} \mu^{-}\right)$:

- Sensitivity to the various deltas:

We find significant sensitivity to the NMFV deltas in scenarios with very large $\tan \beta$, as it is the case of SPS4 and SPS1b. This sensitivity is clearly due to the Higgs-mediated contribution that, grows as $\tan ^{6} \beta$. The largest sensitivity is to $\delta_{23}^{L L}$. In the case of SPS4, there is also significant sensitivity to $\delta_{s b}^{L R}, \delta_{s b}^{R R}$ and $\delta_{c t}^{L R}$. In the SPS1b scenario there is also found some sensitivity to $\delta_{s b}^{L R}, \delta_{c t}^{R R}$, $\delta_{s b}^{R R}$ and $\delta_{c t}^{L R}$.

- Comparing the predictions with the experimental data:

Figure 3 clearly shows that most of the $\left|\delta_{i j}^{X Y}\right| \leq 1$ explored values are allowed by experimental data on $\operatorname{BR}\left(B_{s} \rightarrow \mu^{+} \mu^{-}\right)$. It is in the points with very large $\tan \beta$, i.e SPS4 and SPS1b, where there are some relevant differences between the MFV and the NMFV predictions. First, all predictions for MFV scenarios except for SPS4, are inside the experimental allowed area. In the case of SPS1b, the comparison of the NMFV predictions with data constraints specially $\delta_{23}^{L L}$, but also $\delta_{s b}^{L R}, \delta_{c t}^{R R}, \delta_{s b}^{R R}$ and $\delta_{c t}^{L R}$. In the case of SPS4 some input non-vanishing values of $\delta_{23}^{L L}, \delta_{s b}^{L R}$ or $\delta_{s b}^{R R}$ can place the prediction inside the experimental allowed area. In the case of the SPS1a and SPS3 scenarios some constraints for $\delta_{23}^{L L}$ can be found.

- Intervals of $\delta_{i j}^{X Y}$ allowed by data:

As in the previous observable, we assume here that our predictions for $\operatorname{BR}\left(B_{s} \rightarrow\right.$ $\mu^{+} \mu^{-}$) have a slightly larger error as the SM prediction in (3.12), where, however, the theory uncertainty is very small in comparison with the experimental bound. We choose $\Delta^{\text {theo }}\left(\operatorname{BR}\left(B_{s} \rightarrow \mu^{+} \mu^{-}\right)\right)=0.12 \times 10^{-8}$. Then, a given $\delta_{i j}^{X Y}$ value is allowed by data if the predicted interval, defined by $\operatorname{BR}\left(B_{s} \rightarrow\right.$ $\left.\mu^{+} \mu^{-}\right)+\Delta^{\text {theo }}\left(\operatorname{BR}\left(B_{s} \rightarrow \mu^{+} \mu^{-}\right)\right)$, intersects the experimental area. The upper line of the experimental area in this case is set by the 95\% CL upper bound given in (3.11). It implies that for a predicted ratio to be allowed it must fulfill:

$$
\mathrm{BR}\left(B_{s} \rightarrow \mu^{+} \mu^{-}\right)<1.22 \times 10^{-8} .
$$



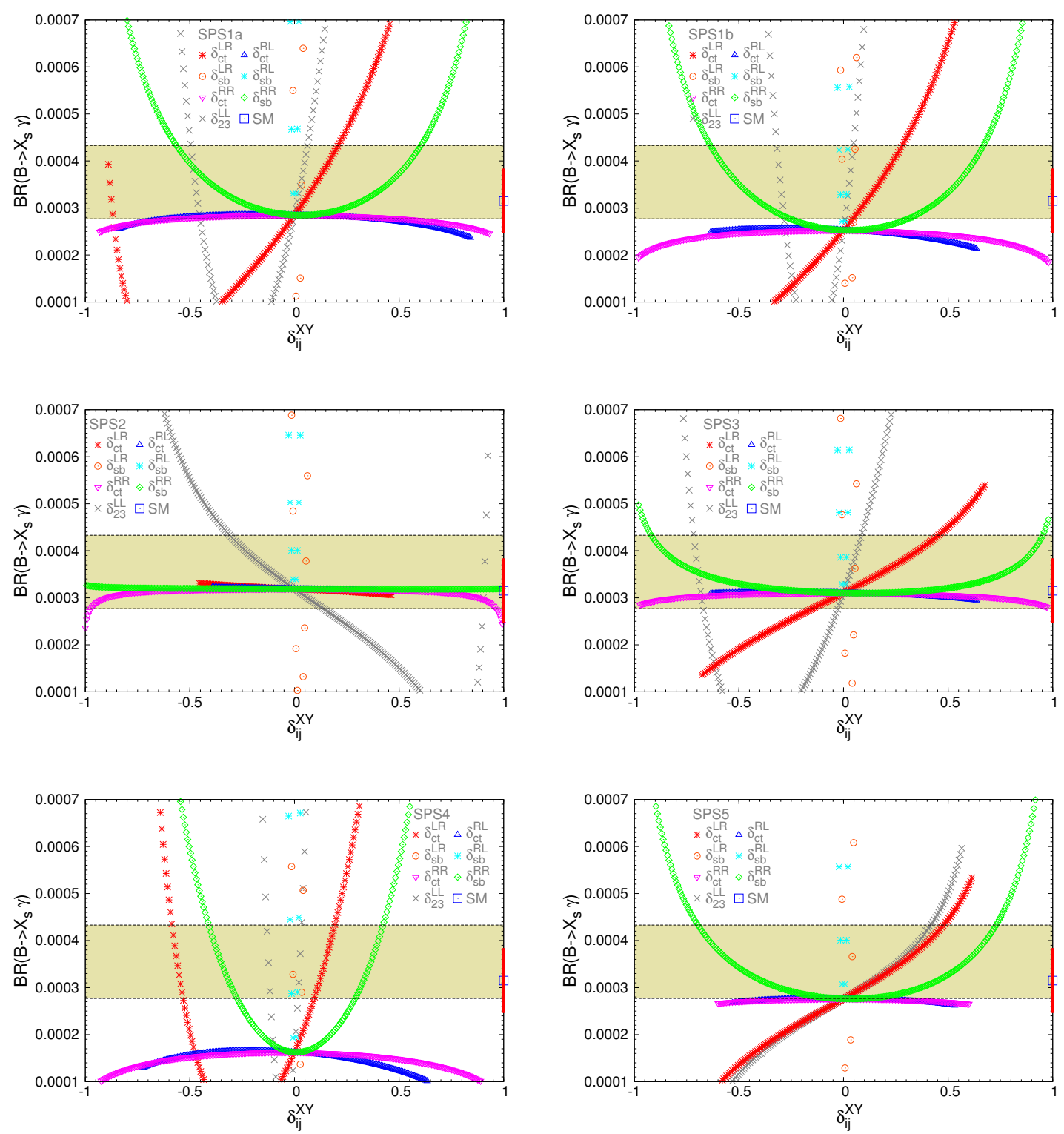

Figure 2. Sensitivity to the NMFV deltas in $\operatorname{BR}\left(B \rightarrow X_{s} \gamma\right)$ for the SPSX points of table 1. The experimental allowed $3 \sigma$ area is the horizontal colored band. The SM prediction and the theory uncertainty $\Delta^{\text {theo }}\left(\mathrm{BR}\left(B \rightarrow X_{s} \gamma\right)\right)$ (red bar) is displayed on the right axis.

The results for the allowed $\delta_{i j}^{X Y}$ intervals are collected in table 2 . We conclude from this table that, except for scenarios with large $\tan \beta \geq 30$, like SPS4 and SPS1b, the size of the deltas can be sizeable, $\left|\delta_{i j}^{X Y}\right|$ larger than $\mathcal{O}(0.1)$, and compatible with $\operatorname{BR}\left(B_{s} \rightarrow \mu^{+} \mu^{-}\right)$data.

- $\Delta M_{B_{s}}$ :

- Sensitivity to the various deltas: 

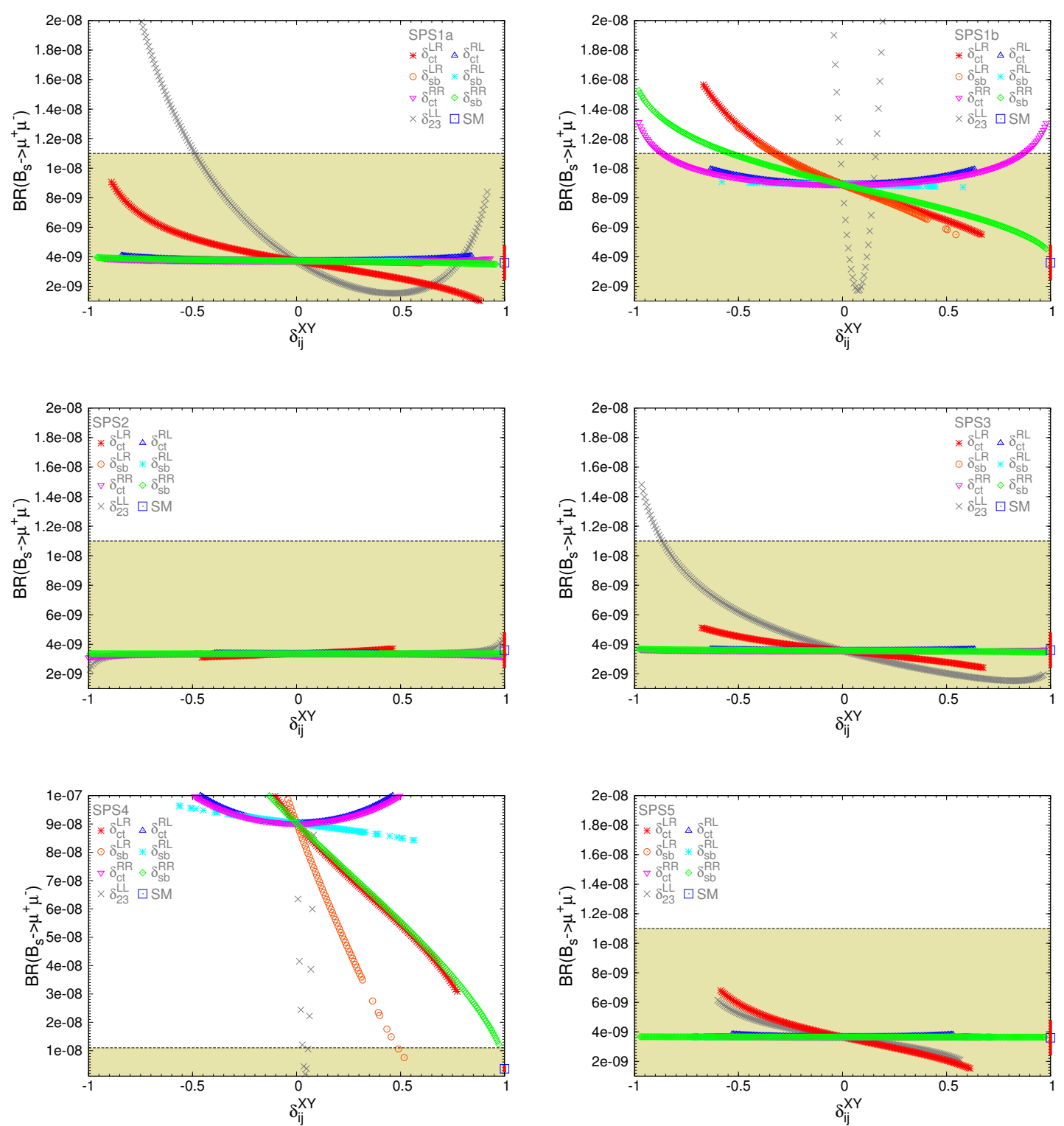

Figure 3. Sensitivity to the NMFV deltas in $\operatorname{BR}\left(B_{s} \rightarrow \mu^{+} \mu^{-}\right)$for the SPSX points of table 1 . The experimental allowed region by the $95 \%$ CL bound is the horizontal colored area. The SM prediction and the theory uncertainty $\Delta^{\text {theo }}\left(\operatorname{BR}\left(B_{s} \rightarrow \mu^{+} \mu^{-}\right)\right)$(red bar) is displayed on the right axis.

Generically, we find strong sensitivity to most of the NMFV deltas in all the studied points, including those with large and low $\tan \beta$ values. The pattern of the $\Delta M_{B_{s}}$ predictions as a function of the various $\delta_{i j}^{X Y}$ differs substantially for each SPS point. This is mainly because in this observable there are two large competing contributions, the double Higgs penguins and the gluino boxes, with very different behavior with $\tan \beta$, as we have seen in figure 1 . In the case of SPS4 with extremely large $\tan \beta=50$, the high sensitivity to all deltas 
is evident in this figure. In the case of SPS5 with low $\tan \beta=5$, there is important sensitivity to all deltas, except $\delta_{c t}^{R R}, \delta_{c t}^{L R}$ and $\delta_{c t}^{R L}$. Generically, for all the studied points, we find the highest sensitivity to 1) $\delta_{s b}^{L R}, \delta_{s b}^{R L}$ and $\delta_{23}^{L L}$; 2) $\delta_{s b}^{R R}$ the next, 3) $\delta_{c t}^{L R}$ the next to next; and 4) the lowest sensitivity is to $\delta_{c t}^{R L}$ and $\delta_{c t}^{R R}$. Consequently, these two later will be the less constrained ones.

- Comparing the predictions with the experimental data:

In this case, the experimental allowed $3 \sigma_{\text {exp }}$ band is very narrow, and all the central predictions at $\delta_{i j}^{X Y}=0$, i.e. for MFV scenarios, lay indeed outside this band. However, if we assume again that our predictions suffer of a similar large uncertainty as the SM prediction, given in (3.21), then the MFV predictions are all compatible with data except for SPS4. It is also obvious from this figure that the predictions within NMFV, as compared to the MFV ones, lie quite generically outside the experimental allowed band, except for the above commented deltas with low sensitivity.

- Intervals of $\delta_{i j}^{X Y}$ allowed by data:

We consider again, that a given $\delta_{i j}^{X Y}$ value is allowed by $\Delta M_{B_{s}}$ data if the predicted interval $\Delta M_{B_{s}} \pm \Delta^{\text {theo }}\left(\Delta M_{B_{s}}\right)$, intersects the experimental band. It corresponds to adding linearly the experimental uncertainty and the theoretical uncertainty. Given the present controversy on the realistic size of the theoretical error in the estimates of $\Delta^{\text {theo }}\left(\Delta M_{B_{s}}\right)$ in the MSSM (see, for instance, [88]), we choose a very conservative value for the theoretical error in our estimates, considerably larger than the SM value in $(3.21)$, of $\Delta^{\text {theo }}\left(\Delta M_{B_{s}}\right)=51 \times 10^{-10} \mathrm{MeV}$. This implies that a predicted mass difference in the interval

$$
63 \times 10^{-10}<\Delta M_{B_{s}}(\mathrm{MeV})<168.6 \times 10^{-10},
$$

is regarded as allowed.

The allowed intervals for the deltas that are obtained with this requirement are collected in table 2. As we have already commented, the restrictions on the b-sector parameters from $\Delta M_{B_{s}}$ are very strong, and in consequence, there are narrow intervals allowed for, $\delta_{s b}^{L R}, \delta_{s b}^{R L}$, and $\delta_{23}^{L L}$. In the case of $\delta_{s b}^{R R}$ there are indeed sequences of very narrow allowed intervals, which in some cases reduce to just a single allowed value. The parameters that show a largest allowed interval, with sizeable $\left|\delta_{i j}^{X Y}\right|$, larger than $\mathcal{O}(0.1)$, are $\delta_{c t}^{R R}, \delta_{c t}^{R L}$ and $\delta_{c t}^{L R}$.

\section{Total allowed $\delta_{i j}^{X Y}$ intervals}

We finally summarize in table 3 the total allowed intervals for all the NMFV deltas, $\delta_{i j}^{X Y}$, where now we have required compatibility with the present data of the three considered $B$ observables, $\operatorname{BR}\left(B \rightarrow X_{s} \gamma\right), \operatorname{BR}\left(B_{s} \rightarrow \mu^{+} \mu^{-}\right)$and $\Delta M_{B_{s}}$. It is obvious, from the previous discussion, that the most restrictive observables are $\operatorname{BR}\left(B \rightarrow X_{s} \gamma\right)$ and $\Delta M_{B_{s}}$, leading to a pattern of allowed delta intervals which is clearly the intersect of their two corresponding intervals. The main conclusion from this table is that, except for SPS4 (the point SPS4 is practically excluded), the NMFV deltas in the top-sector can be sizeable 

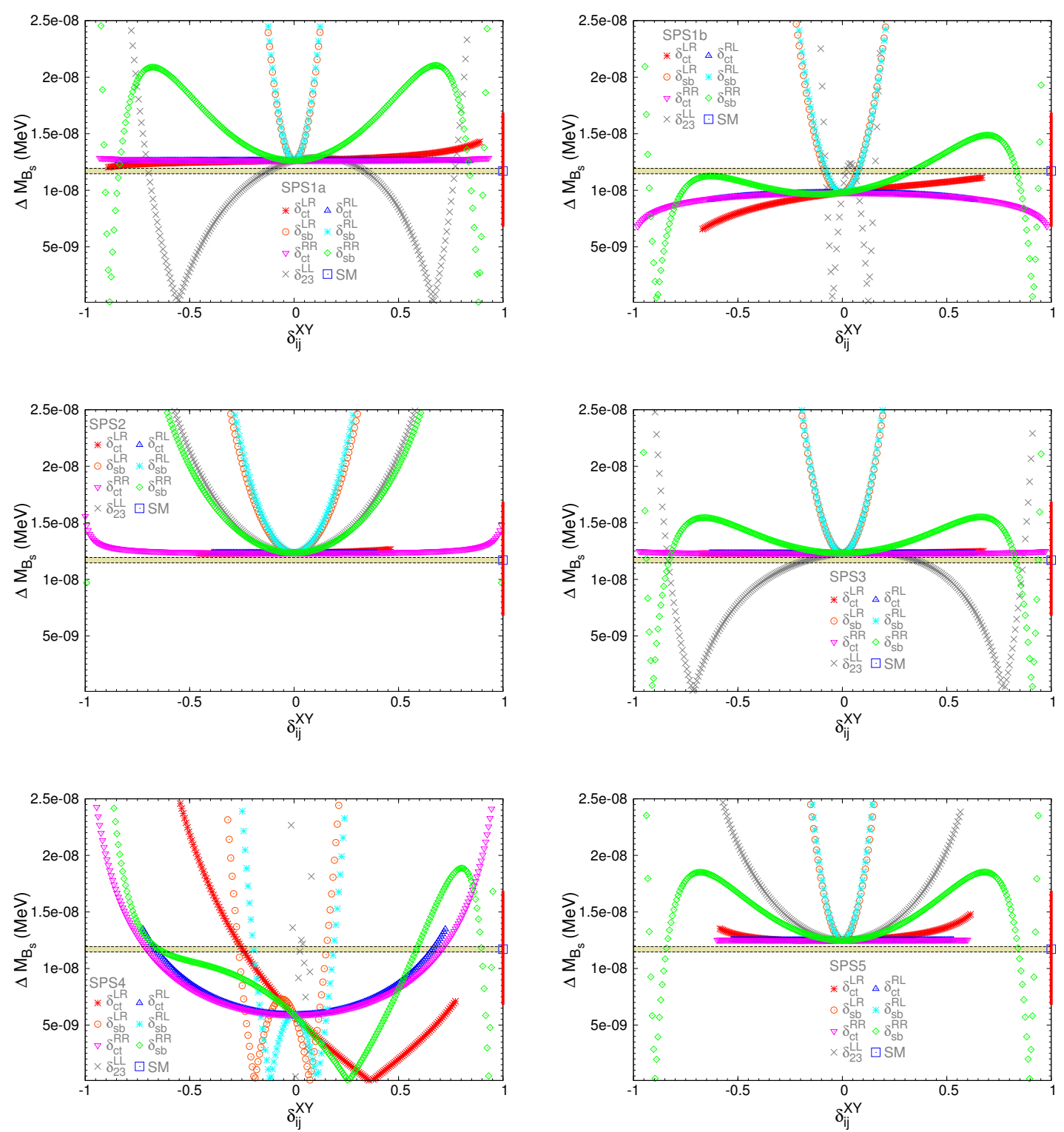

Figure 4. Sensitivity to the NMFV deltas in $\Delta M_{B_{s}}$ for the SPSX points of table 1 . The experimental allowed $3 \sigma_{\exp }$ area is the horizontal colored band. The SM prediction and the theory uncertainty $\Delta^{\text {theo }}\left(\Delta M_{B_{s}}\right)$ (red bar) is displayed on the right axis.

$\left|\delta_{c t}^{X Y}\right|$ larger than $\mathcal{O}(0.1)$ and still compatible with $B$ data. In particular, $\delta_{c t}^{R L}$, and $\delta_{c t}^{R R}$ are the less constrained parameters, and to a lesser extent also $\delta_{c t}^{L R}$. The parameters on the bottom-sector are, in contrast, quite constrained. The most tightly constrained are clearly $\delta_{s b}^{L R}$ and $\delta_{s b}^{R L}$, specially the first one with just some singular allowed values: either positive and of the order of $3-5 \times 10^{-2}$, or negative and with a small size of the order of $-7 \times 10^{-3}$; for the second the limits are around $2 \times 10^{-2}$ for both positive and negative values. $\delta_{s b}^{R R}$ is the less constrained parameter in the bottom sector, with larger allowed intervals of $\left|\delta_{s b}^{R R}\right| \lesssim 0.4-0.9$ depending on the scenario. 
All SPS points are defined with a positive $\mu$ value. We have checked the effect of switching the sign of $\mu$. While the numerical results are changing somewhat, no qualitative change can be observed. Consequently, confining ourselves to positive $\mu$ does not constitute a general restriction of our analysis. Similar observations are made in the Higgs-sector analysis below.

The intervals allowed by $B$ data that we have presented above will be of interest for the following study in this work, where we will next explore the size of the radiative corrections to the MSSM Higgs masses within these NMFV-MSSM scenarios and we will require compatibility with $B$ data. In the final analysis of these corrections, we will use the constraints from $B$ data as extracted from two non-vanishing deltas. As expected, these constraints vary significantly respect to the ones with just one non-vanishing delta.

\section{Radiative corrections to MSSM Higgs masses within NMFV scenarios}

In this section we present our computation of the one-loop radiative corrections to MSSM Higgs boson masses within the NMFV scenarios. For completeness and definiteness, we first shortly review the relevant features of the MSSM Higgs sector at tree-level. Then we summarize the main one-loop renormalization issues that are involved in the computation and finally we present the analytical results for the one-loop corrected Higgs masses.

\subsection{The Higgs boson sector at tree-level}

Contrary to the SM, in the MSSM two Higgs doublets are required. The Higgs potential [89]

$$
\begin{aligned}
V= & m_{1}^{2}\left|\mathcal{H}_{1}\right|^{2}+m_{2}^{2}\left|\mathcal{H}_{2}\right|^{2}-m_{12}^{2}\left(\epsilon_{a b} \mathcal{H}_{1}^{a} \mathcal{H}_{2}^{b}+\text { h.c. }\right) \\
& +\frac{1}{8}\left(g_{1}^{2}+g_{2}^{2}\right)\left[\left|\mathcal{H}_{1}\right|^{2}-\left|\mathcal{H}_{2}\right|^{2}\right]^{2}+\frac{1}{2} g_{2}^{2}\left|\mathcal{H}_{1}^{\dagger} \mathcal{H}_{2}\right|^{2}
\end{aligned}
$$

contains $m_{1}, m_{2}, m_{12}$ as soft SUSY breaking parameters; $g_{2}, g_{1}$ are the $\mathrm{SU}(2)$ and $\mathrm{U}(1)$ gauge couplings, and $\epsilon_{12}=-1$.

The doublet fields $H_{1}$ and $H_{2}$ are decomposed in the following way:

$$
\begin{aligned}
& \mathcal{H}_{1}=\left(\begin{array}{c}
\mathcal{H}_{1}^{0} \\
\mathcal{H}_{1}^{-}
\end{array}\right)=\left(\begin{array}{c}
v_{1}+\frac{1}{\sqrt{2}}\left(\phi_{1}^{0}-i \chi_{1}^{0}\right) \\
-\phi_{1}^{-}
\end{array}\right), \\
& \mathcal{H}_{2}=\left(\begin{array}{c}
\mathcal{H}_{2}^{+} \\
\mathcal{H}_{2}^{0}
\end{array}\right)=\left(\begin{array}{c}
\phi_{2}^{+} \\
v_{2}+\frac{1}{\sqrt{2}}\left(\phi_{2}^{0}+i \chi_{2}^{0}\right)
\end{array}\right) .
\end{aligned}
$$

The potential (4.1) can be described with the help of two independent parameters (besides $g_{1}$ and $\left.g_{2}\right): \tan \beta=v_{2} / v_{1}$ and $M_{A}^{2}=-m_{12}^{2}(\tan \beta+\cot \beta)$, where $M_{A}$ is the mass of the $\mathcal{C P}$-odd Higgs boson $A$. 


\begin{tabular}{|c|c|c|c|c|}
\hline & & $\operatorname{BR}\left(B \rightarrow X_{s} \gamma\right)$ & $\operatorname{BR}\left(B_{s} \rightarrow \mu^{+} \mu^{-}\right)$ & $\Delta M_{B_{s}}$ \\
\hline \multirow{6}{*}{$\delta_{23}^{L L}$} & SPS1a & $(-0.51:-0.43)(-0.034: 0.083)$ & $(-0.53: 0.92)$ & $(-0.73:-0.65)(-0.41: 0.55)(0.73: 0.79)$ \\
\hline & SPS1b & $(-0.33:-0.27)(-0.014: 0.062)$ & $(-0.014: 0.16)$ & $(-0.090:-0.069)(-0.021: 0.097)(0.14: 0.17)$ \\
\hline & SPS2 & $(-0.43: 0.34)(0.90: 0.92)$ & $(-0.99: 0.99)$ & $(-0.37: 0.37)$ \\
\hline & SPS3 & $(-0.73:-0.65)(-0.083: 0.12)$ & $(-0.90: 0.97)$ & $(-0.86:-0.79)(-0.56: 0.66)(0.83: 0.89)$ \\
\hline & SPS4 & $(-0.14:-0.11)(0.0069: 0.034)$ & $(0.028: 0.055)$ & $(-0.0069)(0.021: 0.055)(0.076)$ \\
\hline & SPS5 & $(-0.26: 0.50)$ & $(-0.60: 0.57)$ & $(-0.37: 0.39)$ \\
\hline \multirow{7}{*}{$\delta_{c t}^{L R}$} & SPS1a & $(-0.89:-0.86)(-0.12:-0.097)$ & $(-0.89: 0.89)$ & $(-0.89: 0.89)$ \\
\hline & & $(-0.062: 0.28)$ & & \\
\hline & SPS1b & $(-0.083: 0.36)$ & $(-0.44: 0.67)$ & $(-0.67: 0.67)$ \\
\hline & SPS2 & $(-0.46: 0.46)$ & $(-0.46: 0.46)$ & $(-0.46: 0.46)$ \\
\hline & SPS3 & $(-0.43: 0.61)$ & $(-0.68: 0.68)$ & $(-0.68: 0.68)$ \\
\hline & SPS4 & $(-0.61:-0.51)(0.041: 0.23)$ & excluded & $(-0.39:-0.021)(0.74: 0.77)$ \\
\hline & SPS5 & $(-0.27: 0.58)$ & $(-0.59: 0.61)$ & $(-0.59: 0.61)$ \\
\hline \multirow{6}{*}{$\delta_{s b}^{L R}$} & SPS1a & $(0)(0.034)$ & $(-0.60: 0.60)$ & $(-0.076: 0.076)$ \\
\hline & SPS1b & $(-0.0069: 0)(0.048: 0.055)$ & $(-0.43: 0.54)$ & $(-0.15: 0.14)$ \\
\hline & SPS2 & $(-0.0069: 0)(0.048: 0.055)$ & $(-0.48: 0.48)$ & $(-0.19: 0.19)$ \\
\hline & SPS3 & $(-0.0069: 0)(0.048: 0.055)$ & $(-0.61: 0.61)$ & $(-0.12: 0.12)$ \\
\hline & SPS4 & $(-0.0069)(0.034)$ & $(0.49)$ & $(-0.29:-0.24)(-0.10:-0.014)(0.12: 0.18)$ \\
\hline & SPS5 & $(-0.0069: 0)(0.041)$ & $(-0.71: 0.71)$ & $(-0.090: 0.090)$ \\
\hline \multirow{6}{*}{$\delta_{c t}^{R L}$} & SPS1a & $(-0.84: 0.84)$ & $(-0.84: 0.84)$ & $(-0.84: 0.84)$ \\
\hline & SPS1b & $(-0.63: 0.63)$ & $(-0.63: 0.63)$ & $(-0.63: 0.63)$ \\
\hline & SPS2 & $(-0.39: 0.39)$ & $(-0.39: 0.39)$ & $(-0.39: 0.39)$ \\
\hline & SPS3 & $(-0.63: 0.63)$ & $(-0.63: 0.63)$ & $(-0.63: 0.63)$ \\
\hline & SPS4 & excluded & excluded & $(-0.72:-0.21)(0.21: 0.72)$ \\
\hline & SPS5 & $(-0.53: 0.53)$ & $(-0.53: 0.53)$ & $(-0.53: 0.53)$ \\
\hline \multirow{6}{*}{$\delta_{s b}^{R L}$} & SPS1a & $(-0.014: 0.014)$ & $(-0.71: 0.71)$ & $(-0.069: 0.069)$ \\
\hline & SPS1b & $(-0.021: 0.021)$ & $(-0.58: 0.58)$ & $(-0.14: 0.14)$ \\
\hline & SPS2 & $(-0.014: 0.014)$ & $(-0.55: 0.55)$ & $(-0.17: 0.17)$ \\
\hline & SPS3 & $(-0.021: 0.021)$ & $(-0.63: 0.63)$ & $(-0.11: 0.11)$ \\
\hline & SPS4 & $(-0.021:-0.014)(0.014: 0.021)$ & excluded & $(-0.21:-0.17)(0.16: 0.21)$ \\
\hline & SPS5 & $(-0.014: 0.014)$ & $(-0.72: 0.72)$ & $(-0.083: 0.083)$ \\
\hline \multirow{6}{*}{$\delta_{c t}^{R R}$} & SPS1a & $(-0.93:-0.67)(-0.64: 0.93)$ & $(-0.93: 0.93)$ & $(-0.93: 0.93)$ \\
\hline & SPS1b & $(-0.93:-0.61)(-0.56: 0.90)$ & $(-0.95: 0.94)$ & $(-0.98: 0.98)$ \\
\hline & SPS2 & $(-1.0: 0.99)$ & $(-1.0: 0.99)$ & $(-1.0: 0.99)$ \\
\hline & SPS3 & $(-0.97: 0.97)$ & $(-0.97: 0.97)$ & $(-0.98: 0.97)$ \\
\hline & SPS4 & excluded & excluded & $(-0.85:-0.22)(0.22: 0.85)$ \\
\hline & SPS5 & $(-0.60: 0.60)$ & $(-0.60: 0.60)$ & $(-0.60: 0.60)$ \\
\hline \multirow{6}{*}{$\delta_{s b}^{R R}$} & SPS1a & $(-0.65: 0.68)$ & $(-0.96: 0.96)$ & $\begin{array}{c}(-0.91:-0.90)(-0.86:-0.80)(-0.41: 0.41) \\
(0.81: 0.86)(0.90: 0.91)\end{array}$ \\
\hline & SPS1b & $(-0.71: 0.74)$ & $(-0.73: 0.98)$ & $(-0.94:-0.92)(-0.83: 0.88)(0.93: 0.94)$ \\
\hline & SPS2 & $(-0.99: 0.99)$ & $(-0.99: 0.99)$ & $(-0.99)(-0.39: 0.39)(0.99)$ \\
\hline & SPS3 & $(-0.98: 0.98)$ & $(-0.98: 0.98)$ & $(-0.94:-0.93)(-0.88: 0.88)(0.93: 0.94)$ \\
\hline & SPS4 & $(-0.45:-0.18)(0.19: 0.46)$ & excluded & $(-0.80:-0.028)(0.461: 0.71)(0.86: 0.91)$ \\
\hline & SPS5 & $(-0.77: 0.80)$ & $(-0.97: 0.97)$ & $\begin{aligned}(-0.92) & (-0.87:-0.78)(-0.51: 0.51) \\
& (0.78: 0.87)(0.92)\end{aligned}$ \\
\hline
\end{tabular}

Table 2. Allowed delta intervals by $\mathrm{BR}\left(B \rightarrow X_{s} \gamma\right), \mathrm{BR}\left(B_{s} \rightarrow \mu^{+} \mu^{-}\right)$and $\Delta M_{B_{s}}$. 


\begin{tabular}{|c|c|c|}
\hline & & Total allowed intervals \\
\hline \multirow{6}{*}{$\delta_{23}^{L L}$} & SPS1a & $(-0.034: 0.083)$ \\
\hline & SPS1b & $(-0.014: 0.062)$ \\
\hline & SPS2 & $(-0.37: 0.34)$ \\
\hline & SPS3 & $(-0.083: 0.12)$ \\
\hline & SPS4 & $(0.028: 0.034)$ \\
\hline & SPS5 & $(-0.26: 0.39)$ \\
\hline \multirow{6}{*}{$\delta_{c t}^{L R}$} & SPS1a & $(-0.89:-0.86)(-0.12:-0.097)(-0.062: 0.28)$ \\
\hline & SPS1b & $(-0.083: 0.36)$ \\
\hline & SPS2 & $(-0.46: 0.46)$ \\
\hline & SPS3 & $(-0.43: 0.61)$ \\
\hline & SPS4 & excluded \\
\hline & SPS5 & $(-0.27: 0.58)$ \\
\hline \multirow{6}{*}{$\delta_{s b}^{L R}$} & SPS1a & $(0)(0.034)$ \\
\hline & SPS1b & $(-0.0069: 0)(0.048: 0.055)$ \\
\hline & SPS2 & $(-0.0069: 0)(0.048: 0.055)$ \\
\hline & SPS3 & $(-0.0069: 0)(0.048: 0.055)$ \\
\hline & SPS4 & excluded \\
\hline & SPS5 & $(-0.0069: 0)(0.041)$ \\
\hline \multirow{6}{*}{$\delta_{c t}^{R L}$} & SPS1a & $(-0.84: 0.84)$ \\
\hline & SPS1b & $(-0.63: 0.63)$ \\
\hline & SPS2 & $(-0.39: 0.39)$ \\
\hline & SPS3 & $(-0.63: 0.63)$ \\
\hline & SPS4 & excluded \\
\hline & SPS5 & $(-0.53: 0.53)$ \\
\hline \multirow{6}{*}{$\delta_{s b}^{R L}$} & SPS1a & $(-0.014: 0.014)$ \\
\hline & SPS1b & $(-0.021: 0.021)$ \\
\hline & SPS2 & $(-0.014: 0.014)$ \\
\hline & SPS3 & $(-0.021: 0.021)$ \\
\hline & SPS4 & excluded \\
\hline & SPS5 & $(-0.014: 0.014)$ \\
\hline \multirow{6}{*}{$\delta_{c t}^{R R}$} & SPS1a & $(-0.93:-0.67)(-0.64: 0.93)$ \\
\hline & SPS1b & $(-0.93:-0.61)(-0.56: 0.90)$ \\
\hline & SPS2 & $(-1.0: 0.99)$ \\
\hline & SPS3 & $(-0.97: 0.97)$ \\
\hline & SPS4 & excluded \\
\hline & SPS5 & $(-0.60: 0.60)$ \\
\hline \multirow{6}{*}{$\delta_{s b}^{R R}$} & SPS1a & $(-0.41: 0.41)$ \\
\hline & SPS1b & $(-0.71: 0.74)$ \\
\hline & SPS2 & $(-0.99)(-0.39: 0.39)(0.99)$ \\
\hline & SPS3 & $(-0.94:-0.93)(-0.88: 0.88)(0.93: 0.94)$ \\
\hline & SPS4 & excluded \\
\hline & SPS5 & $(-0.51: 0.51)(0.78: 0.80)$ \\
\hline
\end{tabular}

Table 3. Total allowed delta intervals by $\mathrm{BR}\left(B \rightarrow X_{s} \gamma\right), \mathrm{BR}\left(B_{s} \rightarrow \mu^{+} \mu^{-}\right)$and $\Delta M_{B_{s}}$. 
The diagonalization of the bilinear part of the Higgs potential, i.e. of the Higgs mass matrices, is performed via the orthogonal transformations

$$
\begin{aligned}
\left(\begin{array}{c}
H \\
h
\end{array}\right) & =\left(\begin{array}{cc}
\cos \alpha & \sin \alpha \\
-\sin \alpha & \cos \alpha
\end{array}\right)\left(\begin{array}{c}
\phi_{1}^{0} \\
\phi_{2}^{0},
\end{array}\right) \\
\left(\begin{array}{c}
G \\
A
\end{array}\right) & =\left(\begin{array}{cc}
\cos \beta & \sin \beta \\
-\sin \beta & \cos \beta
\end{array}\right)\left(\begin{array}{l}
\chi_{1}^{0} \\
\chi_{2}^{0}
\end{array}\right), \\
\left(\begin{array}{c}
G^{ \pm} \\
H^{ \pm}
\end{array}\right) & =\left(\begin{array}{cc}
\cos \beta & \sin \beta \\
-\sin \beta & \cos \beta
\end{array}\right)\left(\begin{array}{l}
\phi_{1}^{ \pm} \\
\phi_{2}^{ \pm}
\end{array}\right) .
\end{aligned}
$$

The mixing angle $\alpha$ is determined through

$$
\alpha=\arctan \left[\frac{-\left(M_{A}^{2}+M_{Z}^{2}\right) \sin \beta \cos \beta}{M_{Z}^{2} \cos ^{2} \beta+M_{A}^{2} \sin ^{2} \beta-m_{h, \text { tree }}^{2}}\right], \quad-\frac{\pi}{2}<\alpha<0 .
$$

One gets the following Higgs spectrum:

$$
\begin{aligned}
& 2 \text { neutral bosons, } \mathcal{C P}=+1: h, H \\
& 1 \text { neutral boson, } \mathcal{C P}=-1: A \\
& 2 \text { charged bosons }: H^{+}, H^{-} \\
& 3 \text { unphysical Goldstone bosons }: G, G^{+}, G^{-} .
\end{aligned}
$$

At tree level the mass matrix of the neutral $\mathcal{C P}$-even Higgs bosons is given in the $\phi_{1}-\phi_{2}$-basis in terms of $M_{Z}, M_{A}$, and $\tan \beta$ by

$$
\begin{aligned}
M_{\text {Higgs }}^{2, \text { tree }} & =\left(\begin{array}{cc}
m_{\phi_{1}}^{2} & m_{\phi_{1} \phi_{2}}^{2} \\
m_{\phi_{1} \phi_{2}}^{2} & m_{\phi_{2}}^{2}
\end{array}\right) \\
& =\left(\begin{array}{ccc}
M_{A}^{2} \sin ^{2} \beta+M_{Z}^{2} \cos ^{2} \beta & -\left(M_{A}^{2}+M_{Z}^{2}\right) \sin \beta \cos \beta \\
-\left(M_{A}^{2}+M_{Z}^{2}\right) \sin \beta \cos \beta & M_{A}^{2} \cos ^{2} \beta+M_{Z}^{2} \sin ^{2} \beta
\end{array}\right),
\end{aligned}
$$

which by diagonalization according to eq. (4.3) yields the tree-level Higgs boson masses

$$
M_{\text {Higgs }}^{2, \text { tree }} \stackrel{\alpha}{\longrightarrow}\left(\begin{array}{cc}
m_{H, \text { tree }}^{2} & 0 \\
0 & m_{h, \text { tree }}^{2}
\end{array}\right),
$$

where

$$
\left(m_{H, h}^{2}\right)_{\text {tree }}=\frac{1}{2}\left[M_{A}^{2}+M_{Z}^{2} \pm \sqrt{\left(M_{A}^{2}+M_{Z}^{2}\right)^{2}-4 M_{Z}^{2} M_{A}^{2} \cos ^{2} 2 \beta}\right] .
$$

The charged Higgs boson mass is given by

$$
m_{H^{ \pm}, \text {tree }}^{2}=M_{A}^{2}+M_{W}^{2} .
$$

The masses of the gauge bosons are given in analogy to the SM:

$$
M_{W}^{2}=\frac{1}{2} g_{2}^{2}\left(v_{1}^{2}+v_{2}^{2}\right) ; \quad M_{Z}^{2}=\frac{1}{2}\left(g_{1}^{2}+g_{2}^{2}\right)\left(v_{1}^{2}+v_{2}^{2}\right) ; \quad M_{\gamma}=0 .
$$




\subsection{The Higgs boson sector at one-loop}

In order to calculate one-loop corrections to the Higgs boson masses, the renormalized Higgs boson self-energies are needed. Here we follow the procedure used in refs. [23, 90] (and references therein) and review it for completeness. The parameters appearing in the Higgs potential, see eq. (4.1), are renormalized as follows:

$$
\begin{aligned}
M_{Z}^{2} & \rightarrow M_{Z}^{2}+\delta M_{Z}^{2}, & T_{h} & \rightarrow T_{h}+\delta T_{h}, \\
M_{W}^{2} & \rightarrow M_{W}^{2}+\delta M_{W}^{2}, & T_{H} & \rightarrow T_{H}+\delta T_{H}, \\
M_{\text {Higgs }}^{2} & \rightarrow M_{\text {Higgs }}^{2}+\delta M_{\text {Higgs }}^{2}, & \tan \beta & \rightarrow \tan \beta(1+\delta \tan \beta) .
\end{aligned}
$$

$M_{\text {Higgs }}^{2}$ denotes the tree-level Higgs boson mass matrix given in eq. (4.8). $T_{h}$ and $T_{H}$ are the tree-level tadpoles, i.e. the terms linear in $h$ and $H$ in the Higgs potential.

The field renormalization matrices of both Higgs multiplets can be set up symmetrically,

$$
\left(\begin{array}{l}
h \\
H
\end{array}\right) \rightarrow\left(\begin{array}{cc}
1+\frac{1}{2} \delta Z_{h h} & \frac{1}{2} \delta Z_{h H} \\
\frac{1}{2} \delta Z_{h H} & 1+\frac{1}{2} \delta Z_{H H}
\end{array}\right) \cdot\left(\begin{array}{l}
h \\
H
\end{array}\right)
$$

For the mass counter term matrices we use the definitions

$$
\delta M_{\mathrm{Higgs}}^{2}=\left(\begin{array}{cc}
\delta m_{h}^{2} & \delta m_{h H}^{2} \\
\delta m_{h H}^{2} & \delta m_{H}^{2}
\end{array}\right) .
$$

The renormalized self-energies, $\hat{\Sigma}\left(p^{2}\right)$, can now be expressed through the unrenormalized self-energies, $\Sigma\left(p^{2}\right)$, the field renormalization constants and the mass counter terms. This reads for the $\mathcal{C} \mathcal{P}$-even part,

$$
\begin{aligned}
\hat{\Sigma}_{h h}\left(p^{2}\right) & =\Sigma_{h h}\left(p^{2}\right)+\delta Z_{h h}\left(p^{2}-m_{h, \text { tree }}^{2}\right)-\delta m_{h}^{2}, \\
\hat{\Sigma}_{h H}\left(p^{2}\right) & =\Sigma_{h H}\left(p^{2}\right)+\delta Z_{h H}\left(p^{2}-\frac{1}{2}\left(m_{h, \text { tree }}^{2}+m_{H, \text { tree }}^{2}\right)\right)-\delta m_{h H}^{2}, \\
\hat{\Sigma}_{H H}\left(p^{2}\right) & =\Sigma_{H H}\left(p^{2}\right)+\delta Z_{H H}\left(p^{2}-m_{H, \text { tree }}^{2}\right)-\delta m_{H}^{2} .
\end{aligned}
$$

Inserting the renormalization transformation into the Higgs mass terms leads to expressions for their counter terms which consequently depend on the other counter terms introduced in (4.13).

For the $\mathcal{C P}$-even part of the Higgs sectors, these counter terms are:

$$
\begin{aligned}
\delta m_{h}^{2}= & \delta M_{A}^{2} \cos ^{2}(\alpha-\beta)+\delta M_{Z}^{2} \sin ^{2}(\alpha+\beta) \\
& +\frac{e}{2 M_{Z} s_{\mathrm{w}} c_{\mathrm{w}}}\left(\delta T_{H} \cos (\alpha-\beta) \sin ^{2}(\alpha-\beta)+\delta T_{h} \sin (\alpha-\beta)\left(1+\cos ^{2}(\alpha-\beta)\right)\right) \\
& +\delta \tan \beta \sin \beta \cos \beta\left(M_{A}^{2} \sin 2(\alpha-\beta)+M_{Z}^{2} \sin 2(\alpha+\beta)\right), \\
\delta m_{h H}^{2}= & \frac{1}{2}\left(\delta M_{A}^{2} \sin 2(\alpha-\beta)-\delta M_{Z}^{2} \sin 2(\alpha+\beta)\right) \\
& +\frac{e}{2 M_{Z} s_{\mathrm{w}} c_{\mathrm{w}}}\left(\delta T_{H} \sin ^{3}(\alpha-\beta)-\delta T_{h} \cos ^{3}(\alpha-\beta)\right) \\
& -\delta \tan \beta \sin \beta \cos \beta\left(M_{A}^{2} \cos 2(\alpha-\beta)+M_{Z}^{2} \cos 2(\alpha+\beta)\right), \\
\delta m_{H}^{2}= & \delta M_{A}^{2} \sin ^{2}(\alpha-\beta)+\delta M_{Z}^{2} \cos ^{2}(\alpha+\beta)
\end{aligned}
$$




$$
\begin{aligned}
& -\frac{e}{2 M_{Z} s_{\mathrm{w}} c_{\mathrm{w}}}\left(\delta T_{H} \cos (\alpha-\beta)\left(1+\sin ^{2}(\alpha-\beta)\right)+\delta T_{h} \sin (\alpha-\beta) \cos ^{2}(\alpha-\beta)\right) \\
& -\delta \tan \beta \sin \beta \cos \beta\left(M_{A}^{2} \sin 2(\alpha-\beta)+M_{Z}^{2} \sin 2(\alpha+\beta)\right) .
\end{aligned}
$$

For the field renormalization we chose to give each Higgs doublet one renormalization constant,

$$
\mathcal{H}_{1} \rightarrow\left(1+\frac{1}{2} \delta Z_{\mathcal{H}_{1}}\right) \mathcal{H}_{1}, \quad \mathcal{H}_{2} \rightarrow\left(1+\frac{1}{2} \delta Z_{\mathcal{H}_{2}}\right) \mathcal{H}_{2}
$$

This leads to the following expressions for the various field renormalization constants in eq. (4.14):

$$
\begin{aligned}
\delta Z_{h h} & =\sin ^{2} \alpha \delta Z_{\mathcal{H}_{1}}+\cos ^{2} \alpha \delta Z_{\mathcal{H}_{2}}, \\
\delta Z_{h H} & =\sin \alpha \cos \alpha\left(\delta Z_{\mathcal{H}_{2}}-\delta Z_{\mathcal{H}_{1}}\right), \\
\delta Z_{H H} & =\cos ^{2} \alpha \delta Z_{\mathcal{H}_{1}}+\sin ^{2} \alpha \delta Z_{\mathcal{H}_{2}} .
\end{aligned}
$$

The counter term for $\tan \beta$ can be expressed in terms of the vacuum expectation values as

$$
\delta \tan \beta=\frac{1}{2}\left(\delta Z_{\mathcal{H}_{2}}-\delta Z_{\mathcal{H}_{1}}\right)+\frac{\delta v_{2}}{v_{2}}-\frac{\delta v_{1}}{v_{1}},
$$

where the $\delta v_{i}$ are the renormalization constants of the $v_{i}$ :

$$
v_{1} \rightarrow\left(1+\delta Z_{\mathcal{H}_{1}}\right)\left(v_{1}+\delta v_{1}\right), \quad v_{2} \rightarrow\left(1+\delta Z_{\mathcal{H}_{2}}\right)\left(v_{2}+\delta v_{2}\right)
$$

Similarly for the charged Higgs sector, the renormalized self-energy is expressed in terms of the unrenormalized one and the corresponding counter-terms as:

$$
\hat{\Sigma}_{H^{-} H^{+}}\left(p^{2}\right)=\Sigma_{H^{-} H^{+}}\left(p^{2}\right)+\delta Z_{H^{-} H^{+}}\left(p^{2}-m_{H^{ \pm}, \text {tree }}^{2}\right)-\delta m_{H^{ \pm}}^{2},
$$

where,

$$
\delta m_{H^{ \pm}}^{2}=\delta M_{A}^{2}+\delta M_{W}^{2}
$$

and,

$$
\delta Z_{H^{-} H^{+}}=\sin ^{2} \beta \delta Z_{\mathcal{H}_{1}}+\cos ^{2} \beta \delta Z_{\mathcal{H}_{2}} .
$$

The renormalization conditions are fixed by an appropriate renormalization scheme. For the mass counter terms on-shell conditions are used, resulting in:

$$
\delta M_{Z}^{2}=\operatorname{Re} \Sigma_{Z Z}\left(M_{Z}^{2}\right), \quad \delta M_{W}^{2}=\operatorname{Re} \Sigma_{W W}\left(M_{W}^{2}\right), \quad \delta M_{A}^{2}=\operatorname{Re} \Sigma_{A A}\left(M_{A}^{2}\right) .
$$

For the gauge bosons $\Sigma$ denotes the transverse part of the self-energy. Since the tadpole coefficients are chosen to vanish in all orders, their counter terms follow from $T_{\{h, H\}}+\delta T_{\{h, H\}}=0$ :

$$
\delta T_{h}=-T_{h}, \quad \delta T_{H}=-T_{H} .
$$

For the remaining renormalization constants for $\delta \tan \beta, \delta Z_{\mathcal{H}_{1}}$ and $\delta Z_{\mathcal{H}_{2}}$ the most convenient choice is a $\overline{\mathrm{DR}}$ renormalization of $\delta \tan \beta, \delta Z_{\mathcal{H}_{1}}$ and $\delta Z_{\mathcal{H}_{2}}$,

$$
\delta Z_{\mathcal{H}_{1}}=\delta Z_{\mathcal{H}_{1}}^{\overline{\mathrm{DR}}}=-\left[\operatorname{Re} \Sigma_{H H \mid \alpha=0}^{\prime}\right]^{\mathrm{div}},
$$




$$
\begin{aligned}
\delta Z_{\mathcal{H}_{2}} & =\delta Z_{\mathcal{H}_{2}}^{\overline{\mathrm{DR}}}=-\left[\operatorname{Re} \Sigma_{h h \mid \alpha=0}^{\prime}\right]^{\mathrm{div}}, \\
\delta \tan \beta & =-\frac{1}{2}\left(\delta Z_{\mathcal{H}_{2}}-\delta Z_{\mathcal{H}_{1}}\right)=\delta \tan \beta^{\overline{\mathrm{DR}}} .
\end{aligned}
$$

The corresponding renormalization scale, $\mu_{\overline{\mathrm{DR}}}$, is set to $\mu_{\overline{\mathrm{DR}}}=m_{t}$ in all numerical evaluations.

Finally, in the Feynman diagrammatic (FD) approach that we are following here, the higher-order corrected $\mathcal{C P}$-even Higgs boson masses are derived by finding the poles of the $(h, H)$-propagator matrix. The inverse of this matrix is given by

$$
\left(\Delta_{\text {Higgs }}\right)^{-1}=-i\left(\begin{array}{cc}
p^{2}-m_{H_{\text {tree }}}^{2}+\hat{\Sigma}_{H H}\left(p^{2}\right) & \hat{\Sigma}_{h H}\left(p^{2}\right) \\
\hat{\Sigma}_{h H}\left(p^{2}\right) & p^{2}-m_{h, \text { tree }}^{2}+\hat{\Sigma}_{h h}\left(p^{2}\right)
\end{array}\right) .
$$

Determining the poles of the matrix $\Delta_{\text {Higgs }}$ in eq. (4.28) is equivalent to solving the equation

$$
\left[p^{2}-m_{h, \text { tree }}^{2}+\hat{\Sigma}_{h h}\left(p^{2}\right)\right]\left[p^{2}-m_{H, \text { tree }}^{2}+\hat{\Sigma}_{H H}\left(p^{2}\right)\right]-\left[\hat{\Sigma}_{h H}\left(p^{2}\right)\right]^{2}=0 .
$$

Similarly, in the case of the charged Higgs sector, the corrected Higgs mass is derived by the position of the pole in the charged Higgs propagator, which is defined by:

$$
p^{2}-m_{H^{ \pm}, \text {tree }}^{2}+\hat{\Sigma}_{H^{-} H^{+}}\left(p^{2}\right)=0 .
$$

\subsection{Analytical results of Higgs mass corrections in NMFV-SUSY}

Following the previously detailed prescription for the computation of the one-loop corrected Higgs boson masses, one finds the analytical results for these masses in terms of the renormalized self-energies which, in turn, are written in terms of the unrenormalized self-energies and tadpoles. To shorten the presentation of these analytical results, it is convenient to report just on these unrenormalized self-energies and tadpoles.

The relevant one-loop corrections have been evaluated with the help of FeynArts [5052] and FormCalc [53]. For completeness the new Feynman rules included in the model file are listed in the appendix A. All the results for the unrenormalized self-energies and tadpoles are collected in appendix B. We have shown explicitly just the relevant contributions for the present study of the radiative corrections to the Higgs boson masses within NMFV scenarios, namely, the one-loop contributions from quarks and squarks. The corresponding generic Feynman-diagrams for the Higgs bosons self-energies, gauge boson self-energy diagrams and tadpole diagrams are collected in the figure 15 in appendix B. It should also be noticed that the contributions from the squarks are the only ones that differ from the usual ones of the MSSM with MFV. It should be noted also that the corrections from flavor mixing, which are the subject of our interest here, are implicit in both the $V_{\mathrm{CKM}}$, and in the values of the rotation matrices, $R^{\tilde{u}}, R^{\tilde{d}}$, and the squark masses, $m_{\tilde{u}_{i}}, m_{\tilde{d}_{i}}(i=1, \ldots, 6)$ that appear in these formulas of the unrenormalized self-energies and tadpoles and that have been introduced in section 2 .

Finally, it is worth mentioning that we have checked the finiteness in our analytical results for the renormalized Higgs self-energies. It is obviously expected, but it is not a 
trivial check in the present scenarios with three generations of quarks and squarks and with flavor mixing. We have also checked that the analytical results of the self-energies in appendix B agree with the formulas in FeynHiggs [23, 24, 29-31]. Each one of the terms contained in the appendix B was compared with the corresponding term in FeynHiggs. During this process and the check of the finiteness, discrepancies were found with the charged Higgs part of FeynHiggs, leading to an updated version of the code. ${ }^{10}$

\section{$5 \quad$ Numerical analysis}

In this section we present our numerical results for the radiative corrections to the Higgs boson masses from from flavor mixing within NMFV-SUSY scenarios. Since all one-loop corrections in the present NMFV scenario are common to the MSSM except for the corrections from squarks, which depend on the $\delta_{i j}^{X Y}$ values, we will focus just on the results of these corrections as a function of the flavor mixing parameters, and present the differences with respect to the predictions within the MSSM. Correspondingly, we define:

$$
\Delta m_{\phi}\left(\delta_{i j}^{X Y}\right) \equiv m_{\phi}^{\mathrm{NMFV}}\left(\delta_{i j}^{X Y}\right)-m_{\phi}^{\mathrm{MSSM}}, \quad \phi=h, H, H^{ \pm},
$$

where $m_{\phi}^{\mathrm{NMFV}}\left(\delta_{i j}^{X Y}\right)$ and $m_{\phi}^{\mathrm{MSSM}}$ have been calculated at the one-loop level. It should be noted that $m_{\phi}^{\mathrm{NMFV}}\left(\delta_{i j}^{X Y}=0\right)=m_{\phi}^{\mathrm{MSSM}}$ and, therefore, by construction, $\Delta m_{\phi}\left(\delta_{i j}^{X Y}=0\right)=$ 0 , and $\Delta m_{\phi}$ gives the size of the one-loop NMFV contributions to $m_{\phi}$. The numerical calculation of $m_{\phi}^{\mathrm{NMFV}}\left(\delta_{i j}^{X Y}\right)$ and $m_{\phi}^{\mathrm{MSSM}}$ has been done with (the updated version of) FeynHiggs [23, 24, 29-31], which solves the eqs. (4.29) and (4.30) for finding the positions of the poles of the propagator matrix. Previous results for $\Delta m_{h}\left(\delta_{23}^{L L}\right)$ can be found in [28].

\section{1 $\Delta m_{\phi}$ versus one $\delta_{i j}^{X Y} \neq 0$}

We show in figures 5, 6 and 7 our numerical results for $\Delta m_{h}, \Delta m_{H}$ and $\Delta m_{H^{ \pm}}$, respectively, as functions of the seven considered flavor changing deltas, $\delta_{23}^{L L}, \delta_{c t}^{L R}, \delta_{s b}^{L R}$, $\delta_{c t}^{R L}, \delta_{s b}^{R L}, \delta_{c t}^{R R}$ and $\delta_{s b}^{R R}$, which we vary in the interval $-1 \leq \delta_{i j}^{X Y} \leq 1$. In these plots we have chosen the same six SPS points of table 1, as for the previous study of constraints from $B$ physics in 2 . We do not take the experimental bounds into account here, since we just want to show the general behavior of the masses with the deltas. The experimental bounds will be taken into account in the next subsection. As before we have checked the impact of switching the sign of $\mu$ and found a small quantitative but no qualitative effect.

The main conclusions from these figures are the following:

- General features:

All mass corrections, $\Delta m_{h}, \Delta m_{H}$ and $\Delta m_{H^{ \pm}}$, are symmetric $\delta_{i j}^{X Y} \rightarrow-\delta_{i j}^{X Y}$, as expected. This feature is obviously different than in the previous plots of the $B$ observables. The sign of the mass corrections can be both positive and negative, depending on the particular delta value. The size of the Higgs mass corrections, can be very large in some $\delta_{i j}^{X Y} \neq 0$ regions, reaching values even larger than $10 \mathrm{GeV}$ in

\footnotetext{
${ }^{10}$ We especially thank T. Hahn for his efforts put into this update.
} 
some cases, at the central region with not very large delta values, $\left|\delta_{i j}^{X Y}\right|<0.5$. In fact, the restrictions from $B$ physics in this central region is crucial to get a reliable estimate of these effects.

For low $\tan \beta$, where the restrictions from $B$ physics to the deltas are less severe, the Higgs mass corrections are specially relevant. Particularly, $\Delta m_{h}$ turns out to be negative and large for $\tan \beta=5$ (SPS5) for all deltas, except $\delta_{s b}^{R R}$. For instance, at $\left|\delta_{i j}^{X Y}\right| \simeq 0.5$, the mass correction $\Delta m_{h}$ for SPS5 is negative and $\gtrsim 5 \mathrm{GeV}$ in all flavor changing deltas except $\delta_{s b}^{R R}$ where the correction is negligible. In the case of $\Delta m_{H}$ and $\Delta m_{H^{ \pm}}$the size of the correction at low $\tan \beta$ is smaller, $\lesssim 2 \mathrm{GeV}$ in the central region, except for $\delta_{s b}^{L R}$ and $\delta_{s b}^{R L}$ that can also generate large corrections $\gtrsim 5 \mathrm{GeV}$.

In the cases with large $\tan \beta$ (SPS4 and SPS1b), we also find large mass corrections but, as already said, they are much more limited by $B$ constraints. In particular, for SPS4 all deltas are excluded, except for a very narrow window in $\delta_{23}^{L L}$ (see table 3 ).

In the cases with moderate $\tan \beta=10$ (SPS1a, SPS2 and SPS3), we find large corrections $\left|\Delta m_{h}\right| \gtrsim 5 \mathrm{GeV}$ in the central region of $\delta_{s b}^{L R}, \delta_{s b}^{R L}, \delta_{c t}^{L R}$ and $\delta_{c t}^{R L}$. The other Higgs bosons get large corrections $\left|\Delta m_{H}\right|,\left|\Delta m_{H}\right| \gtrsim 5 \mathrm{GeV}$ in the deltas central region only for $\delta_{s b}^{L R}$ and $\delta_{s b}^{R L}$.

- Sensitivity to the various deltas:

We find very strong sensitivity in the three mass corrections $\Delta m_{h}, \Delta m_{H}$ and $\Delta m_{H^{ \pm}}$, to $\delta_{s b}^{L R}$ and $\delta_{s b}^{R L}$ for all the seven considered SPS points.

In the case of $\Delta m_{h}$ there is also an important sensitivity to $\delta_{c t}^{L R}$ and $\delta_{c t}^{R L}$ in all the considered points. The strong sensitivity to $L R$ and $R L$ parameters can be understood due to the relevance of the $A$-terms in these Higgs mass corrections. It can be noticed in the Feynman rules (i.e. see the coupling of two squarks and one/two Higgs bosons in appendix A) that the $A$-terms enter directly into the couplings, and in some cases, as in the couplings of down-type squarks to the CP-odd Higgs boson, enhanced by $\tan \beta$. Therefore, considering the relationship between the $A$-terms and these $L R$ and $R L$ parameters as is shown in eq. (2.24), the strong sensitivity to these parameters can be understood. A similar strong sensitivity to $\delta_{c t}^{L R}$ in $\Delta m_{h}$ has been found in [37].

In SPS5 there is a noticeable sensitivity to all deltas except $\delta_{s b}^{R R}$. In other points, the effects of $\delta_{23}^{L L}, \delta_{c t}^{R R}$ on $\Delta m_{h}$ are only appreciated at the large delta region, close to \pm 1 . For instance, in SPS2, $\Delta m_{h}=-5 \mathrm{GeV}$ for $\delta_{c t}^{R R}= \pm 1$.

In the case of $\Delta m_{H}$, apart from $\delta_{s b}^{L R}$ and $\delta_{s b}^{R L}$, there is only noticeable sensitivity to other deltas in SPS5. The same comment applies to $\Delta m_{H^{ \pm}}$.

\section{$5.2 \Delta m_{h}$ versus two $\delta_{i j}^{X Y} \neq 0$}

Our previous results on the Higgs mass corrections show that the corrections to the lightest Higgs mass $\Delta m_{h}$ are negative in many of the studied cases and can be very large for some regions of the flavor changing deltas which are still allowed by present $B$ data. These 

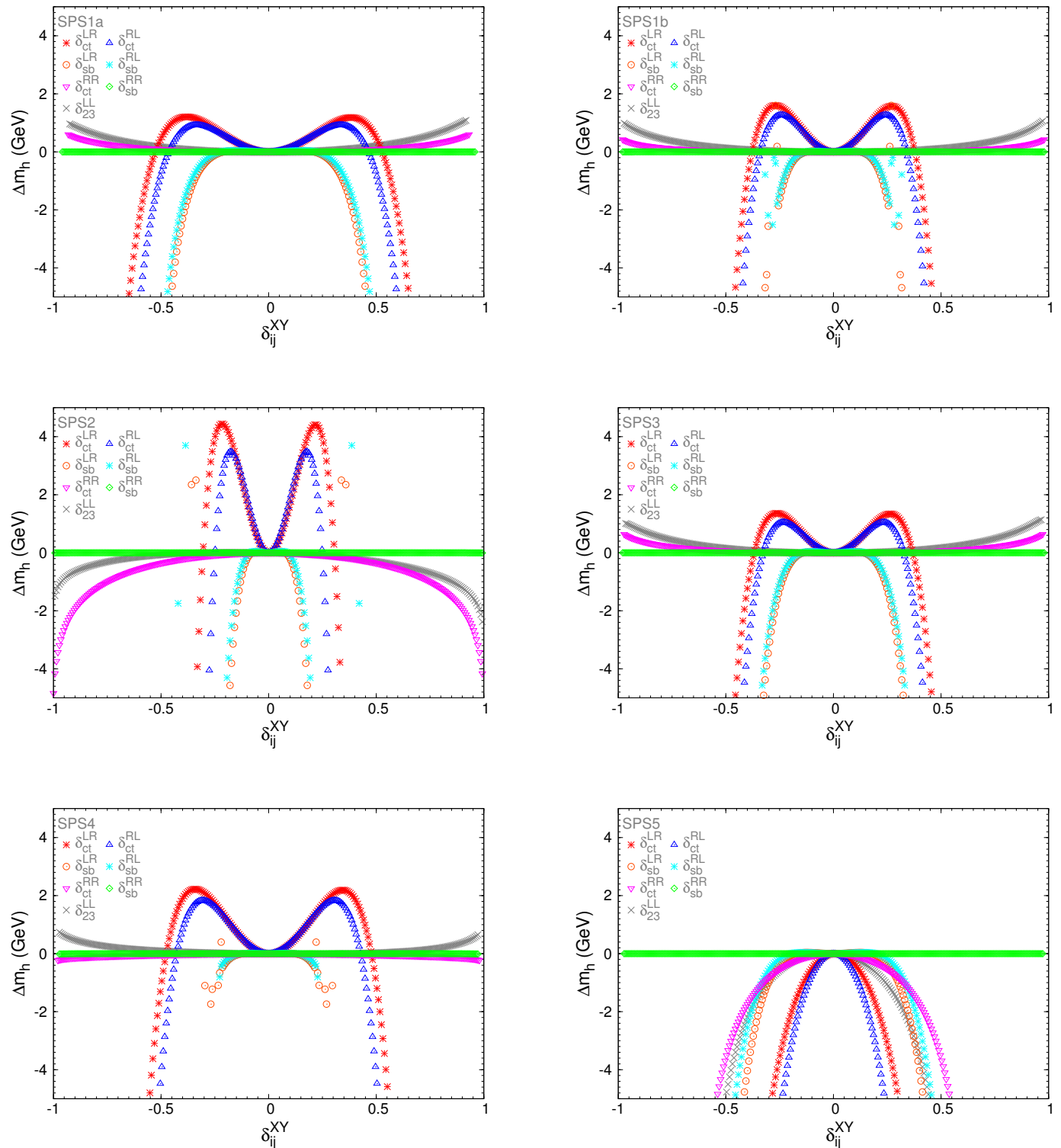

Figure 5. Sensitivity to the NMFV deltas in $\Delta m_{h}$ for the SPSX points of table 1.

negative and large mass corrections, can lead to a prediction for the corrected one-loop mass in these kind of NMFV-SUSY scenarios, $m_{h}^{\mathrm{NMFV}} \simeq m_{h}^{\mathrm{MSSM}}+\Delta m_{h}$, which are indeed too low and already excluded by present data $[9,10]$. Therefore, interestingly, the study of these mass corrections can be conclusive in the setting of additional restrictions on the size of some flavor changing deltas which otherwise are not bounded from present $B$ data.

In order to explore further the size of these 'dangerous' mass corrections, we have computed numerically the size of $\Delta m_{h}$ as a function of two non-vanishing deltas and have looked for areas in these two dimensional plots that are allowed by $B$ data. We show in figures $9,10,11,12,13$, and 14 the numerical results of the $\Delta m_{h}$ contour-lines in two 

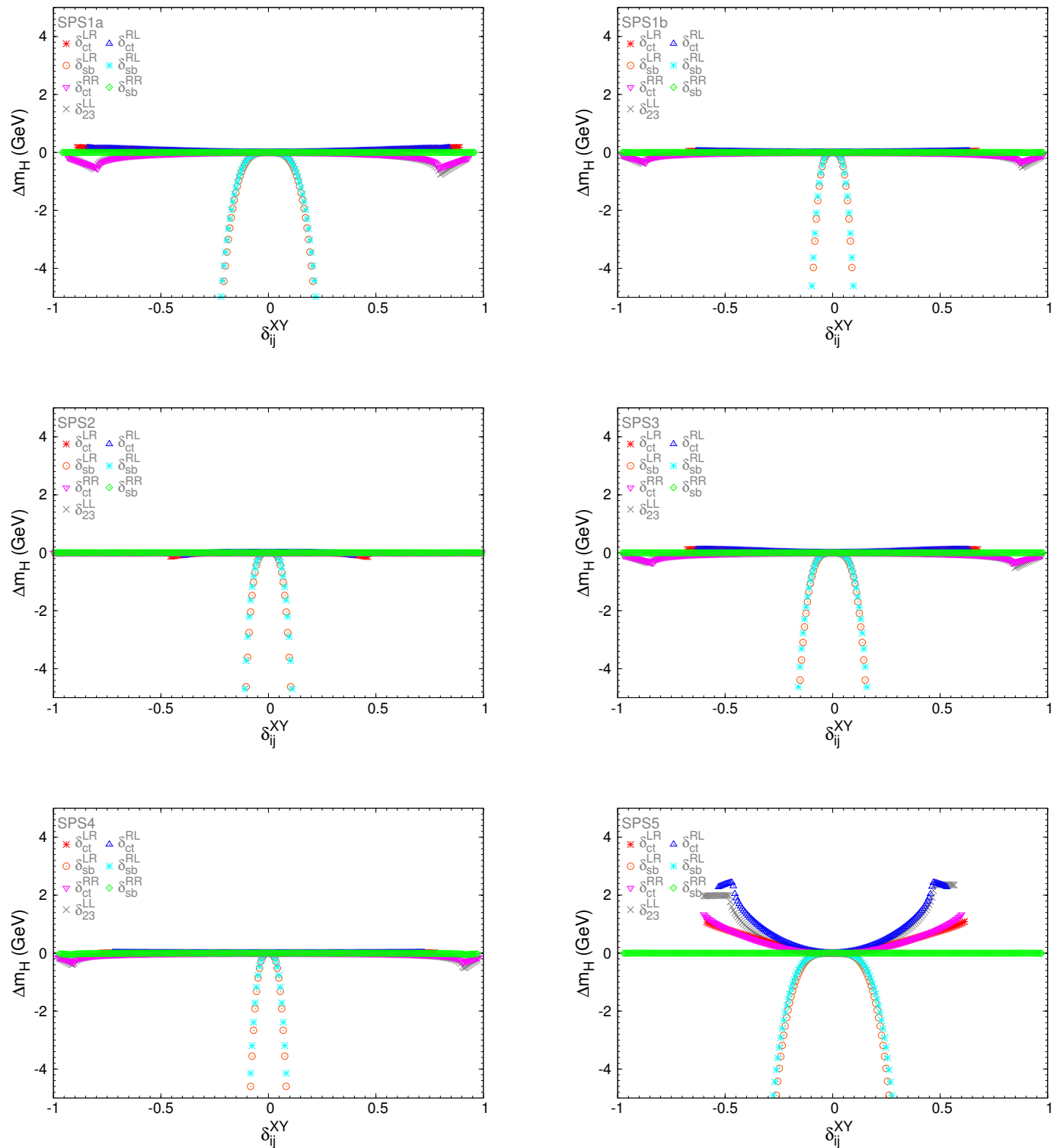

Figure 6. Sensitivity to the NMFV deltas in $\Delta m_{H}$ for the SPSX points of table 1.

dimensional plots, $\left(\delta_{23}^{L L}, \delta_{i j}^{X Y}\right)$, for the respective points BFP, SPS2, SPS3, SPS5, VHeavyS and HeavySLightH of table 1.

We have chosen in all plots $\delta_{23}^{L L}$ as one of these non-vanishing deltas mainly because of two reasons. First, because it is one of the most frequently studied flavor changing parameters in the literature and, therefore, a convenient reference parameter. Second, because there are several well motivated SUSY scenarios, where this parameter gets the largest value, as we explained in section 2 .

In these two-dimensional figures we have included the allowed/disallowed by $B$ data areas that have been found by following the procedure explained in section 3 , and the 

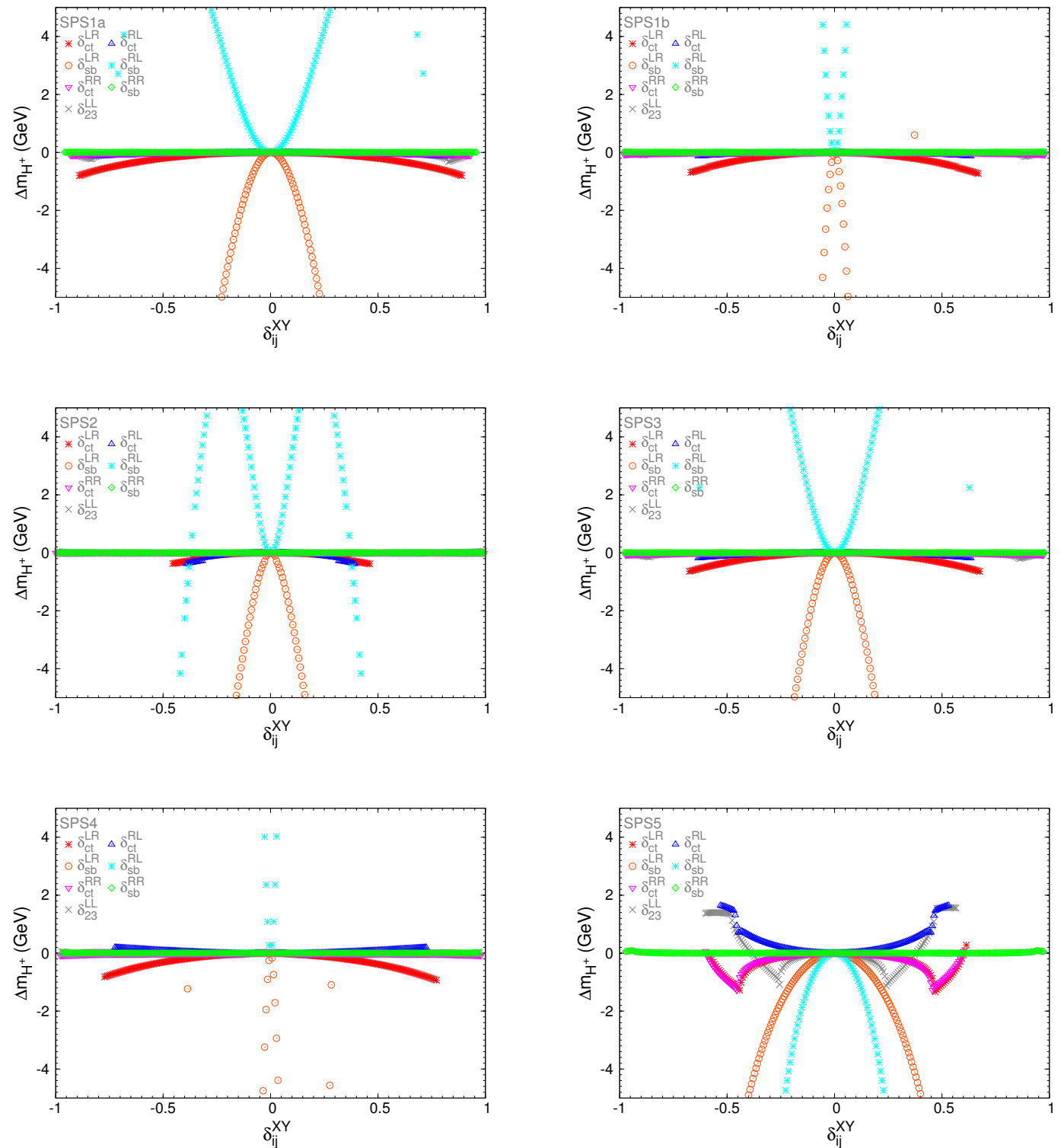

Figure 7. Sensitivity to the NMFV deltas in $\Delta m_{H^{ \pm}}$for the SPSX points of table 1.

allowed intervals are given in eqs. (3.22), (3.23) and (3.24). The color code explaining the meaning of each colored area and the codes for the discontinuous lines are given in figure 8. Contour lines corresponding to mass corrections above $60 \mathrm{GeV}$ or below $-60 \mathrm{GeV}$ have not been represented. In several scenarios the plots involving $\delta_{s b}^{L R, R L}$ show a seemingly abrupt behavior for $\left|\delta_{s b}^{L R, R L}\right| \gtrsim 0.3$, corresponding to extremely large (one-loop) corrections to $m_{h}$. In general, in the case of very large one-loop corrections, in order to get a more stable result further higher order corrections would be required, as it is known from the higher-order corrections to $m_{h}$ in the MFV case (see, e.g., ref. [24]). However, we cannot explore this possibility here. On the other hand, in order to understand the behavior of 


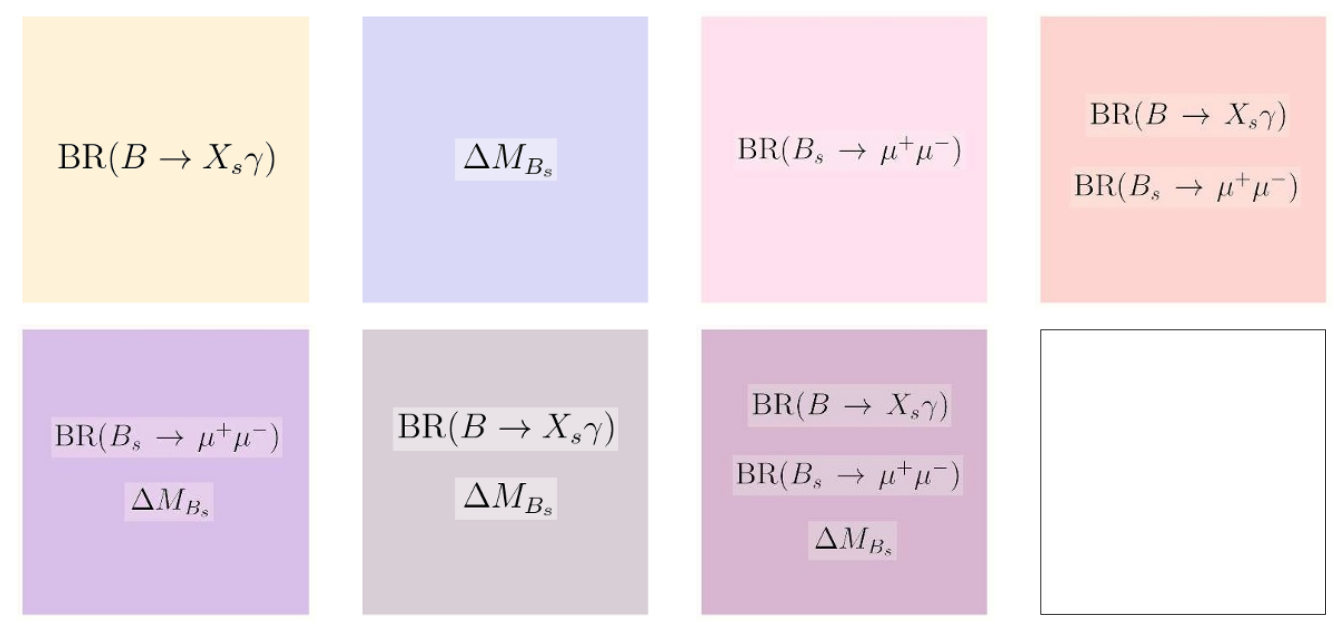

Figure 8. Legend for plots of Higgs mass corrections varying two deltas simultaneously displayed in figures 9, 10, 11, 12, 13 and 14. Each colored area represents the disallowed region by the specified observable/s inside each box. A white area placed at the central regions of the mentioned figures represents a region allowed by the three $B$ observables. A white area placed outside the colored areas represent regions of the parameter space that generate negative squared masses. These problematic points are consequently not shown in our plots, as we did in the previous plots. The discontinuous lines in those figures represent the contour lines for the $B$ observables corresponding to the maximum and minimum allowed values: dash-dot-dash for the upper bound of $\operatorname{BR}\left(B \rightarrow X_{s} \gamma\right)$ (eq. (3.22)), dot-dash-dot for the lower bound of $\operatorname{BR}\left(B \rightarrow X_{s} \gamma\right)$ (eq. (3.22)), dashed line for the upper bound of $\Delta M_{B_{s}}$ (eq. (3.24)), a sequential three dotted line for the lower bound of $\Delta M_{B_{s}}$ (eq. (3.24)), and a dotted line for the upper bound of $\operatorname{BR}\left(B_{s} \rightarrow \mu^{+} \mu^{-}\right)$(eq. (3.23)).

$m_{h}$ as a function of $\delta_{s b}^{L R, R L}$ a simple analytical formula would have to be extracted from the general result. However, this is beyond the scope of our paper.

The main conclusions from these two dimensional figures are summarized in the following:

The points that have been chosen in these plots are quite representative of all the different patterns found. The plots for SPS1a (not shown here) manifest similar patterns as those of SPS3. The plots for SPS1b (not shown here) manifest similar patterns as those of BFP. The plots for SPS4 are not included because they do not manifest any allowed areas by $B$ data.

The largest mass corrections $\Delta m_{h}$ found, being allowed by $B$ data occur in plots $\left(\delta_{23}^{L L}, \delta_{c t}^{L R}\right)$ and $\left(\delta_{23}^{L L}, \delta_{c t}^{R L}\right)$. This applies to all the studied points. They can be as large as $(-50,-100) \mathrm{GeV}$ at $\delta_{c t}^{L R}$ or $\delta_{c t}^{R L}$ close to the upper and lower horizontal bands in these plots where $\delta_{c t}^{L R}$ or $\delta_{c t}^{R L}$ are close to \pm 0.5 . Again these large corrections from the $L R$ and $R L$ parameters are due to the $A$-terms, as we explained at the end of section 5.1. Comparing the different plots, it can be seen that the size of the allowed area by the $B$ data (the white area inside of the colored regions) can be easily understood basically in terms of $\tan \beta$, and the heaviness of the SUSY and Higgs spectra. Generically, the plots with largest allowed regions and with largest Higgs mass corrections correspond to scenarios with low $\tan \beta=5$ and heavy spectra. Consequently, the cases of VHeavyS and 

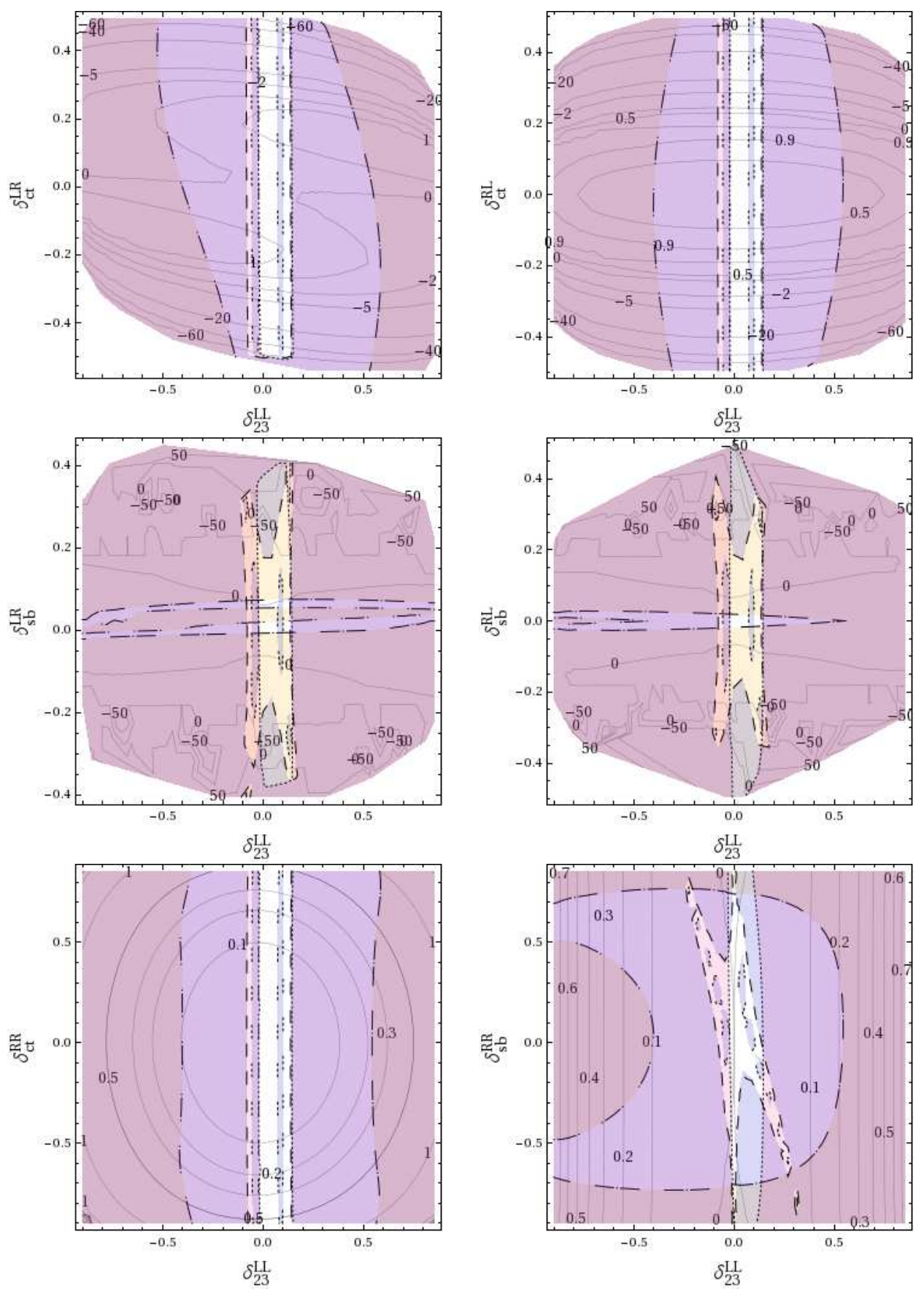

Figure 9. $\Delta m_{h}(\mathrm{GeV})$ contour lines from our two deltas analysis for BFP. The color code for the allowed/disallowed areas by $B$ data is given in figure 8 .

HeavySLightH are the most interesting ones, exhibiting very large radiative corrections, resulting from the heavy SUSY spectra. In the case of HeavySLightH the large corrections are not only found for $\Delta m_{h}$, but also, though to a lesser extent, for the other Higgs bosons, $\Delta m_{H}$ and $\Delta m_{H^{ \pm}}$(not shown here). Consequently, in this scenario the deltas will be very restricted by the mass bounds, especially by $m_{h}$. 

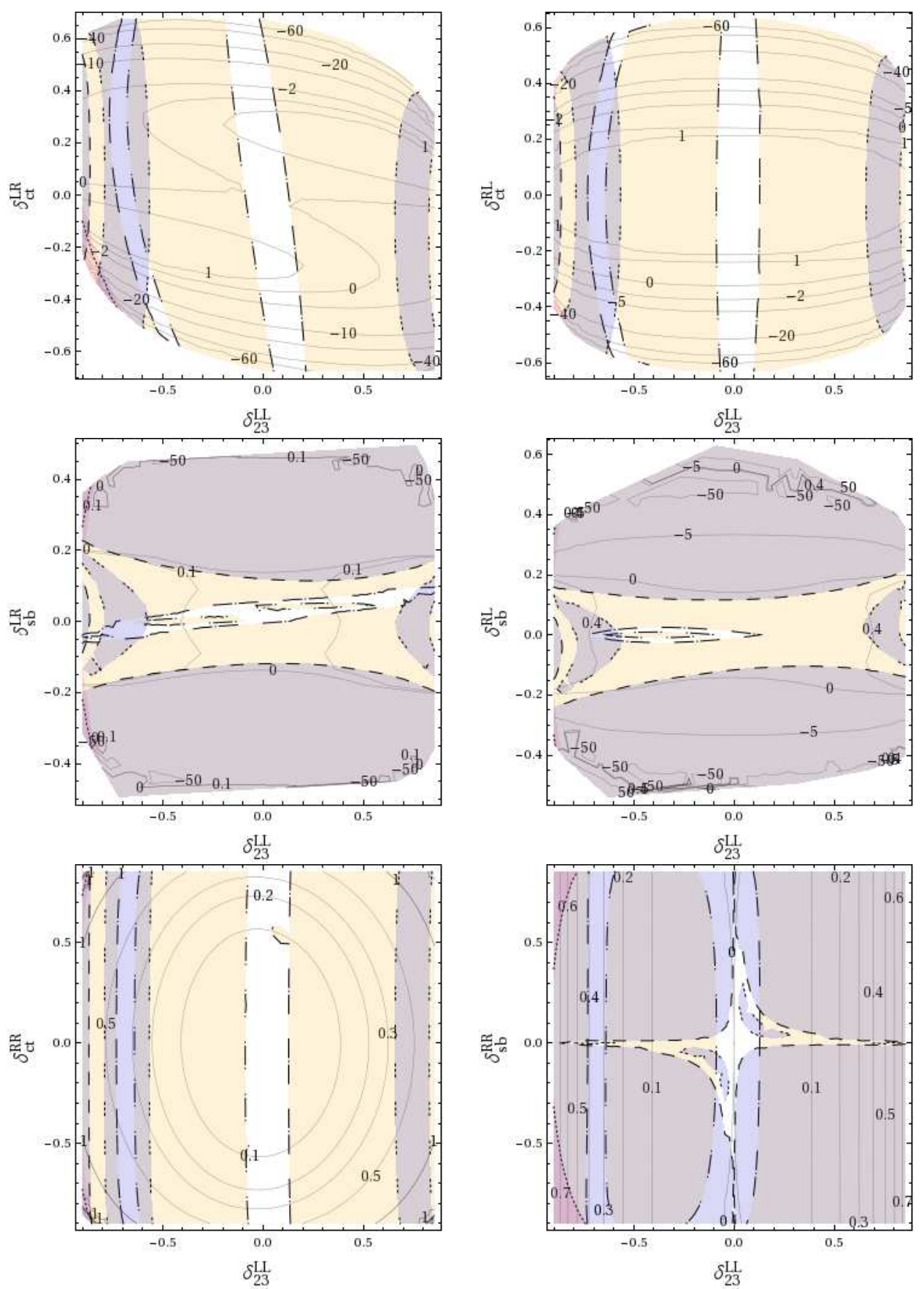

Figure 10. $\Delta m_{h}(\mathrm{GeV})$ contour lines from our two deltas analysis for SPS3. The color code for the allowed/disallowed areas by $B$ data is given in figure 8 .

There are also important corrections in the allowed areas of the two dimensional plots of $\left(\delta_{23}^{L L}, \delta_{c t}^{R R}\right)$ for some points, particularly for SPS5 (and to a lesser extent for SPS2). Here the corrections can be as large as $-50 \mathrm{GeV}$ in the upper and lower parts, i.e. for $\delta_{c t}^{R R}$ close to \pm 0.5 . In the case of SPS2 they can be up to $-2 \mathrm{GeV}$ for this same region.

As for the remaining two-dimensional plots they do not show relevant allowed areas where the mass corrections are interestingly large. 

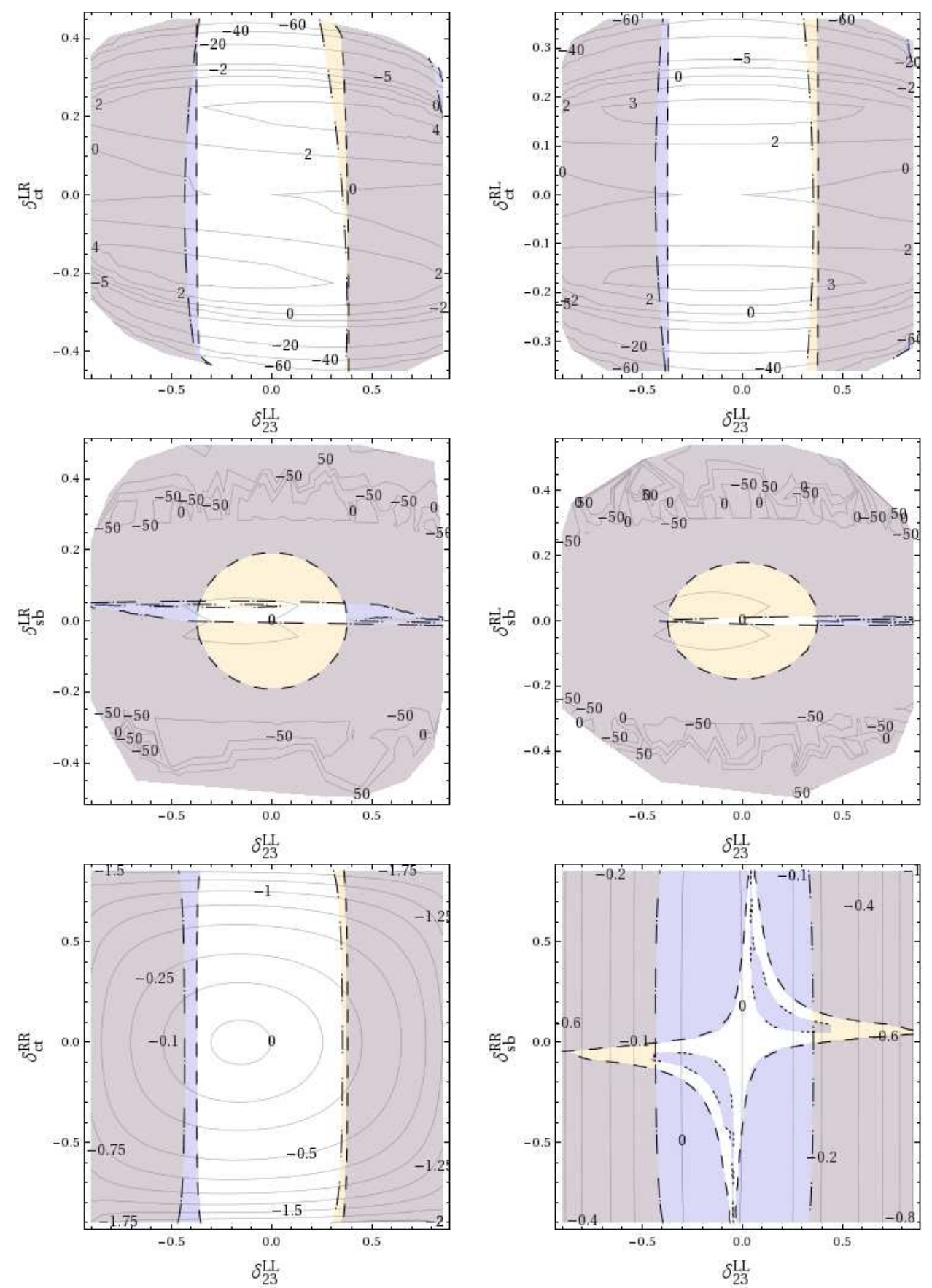

Figure 11. $\Delta m_{h}(\mathrm{GeV})$ contour lines from our two deltas analysis for SPS2. The color code for the allowed/disallowed areas by $B$ data is given in figure 8 .

\section{Conclusions}

In this paper we have analyzed the one-loop corrections to the Higgs boson masses in the MSSM with Non-Minimal Flavor Violation. We assume the flavor violation is being generated from the hypothesis of general flavor mixing in the squark mass matrices, and these are parametrized by a complete set of $\delta_{i j}^{X Y}(X, Y=L, R ; i, j=t, c, u$ or $b, s, d)$. 

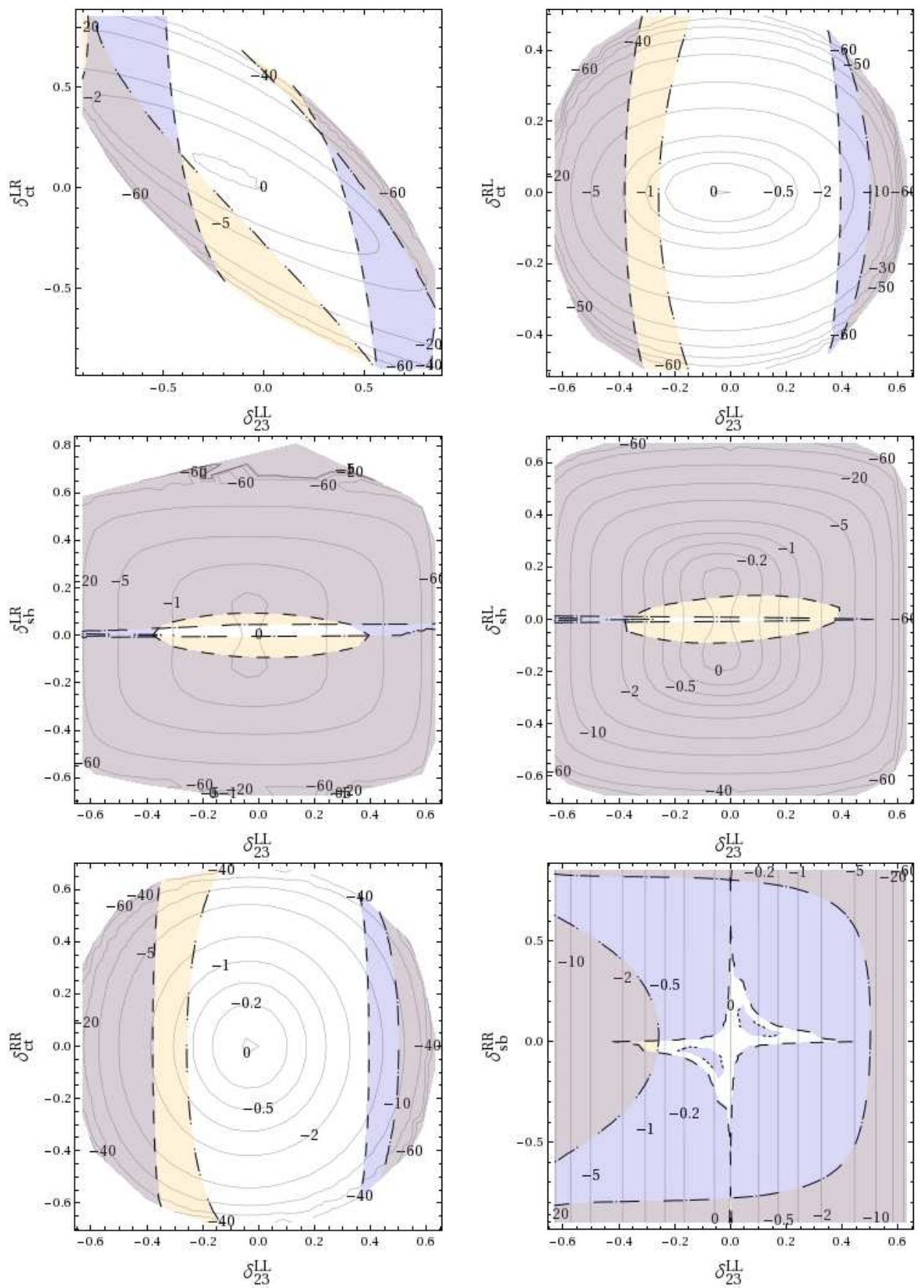

Figure 12. $\Delta m_{h}(\mathrm{GeV})$ contour lines from our two deltas analysis for SPS5. The color code for the allowed/disallowed areas by $B$ data is given in figure 8 .

In the first step of the analysis we scanned over the NMFV parameters, contrasting them with the experimental bounds on $\mathrm{BR}\left(B \rightarrow X_{s} \gamma\right), \mathrm{BR}\left(B_{s} \rightarrow \mu^{+} \mu^{-}\right)$and $\Delta M_{B_{s}}$. We take into account the most up-to-date evaluations in the NMFV MSSM for $\operatorname{BR}\left(B \rightarrow X_{s} \gamma\right)$, $\operatorname{BR}\left(B_{s} \rightarrow \mu^{+} \mu^{-}\right)$and $\Delta M_{B_{s}}$, as included in the BPHYSICS subroutine of the SuFla code $[42,43]$. 

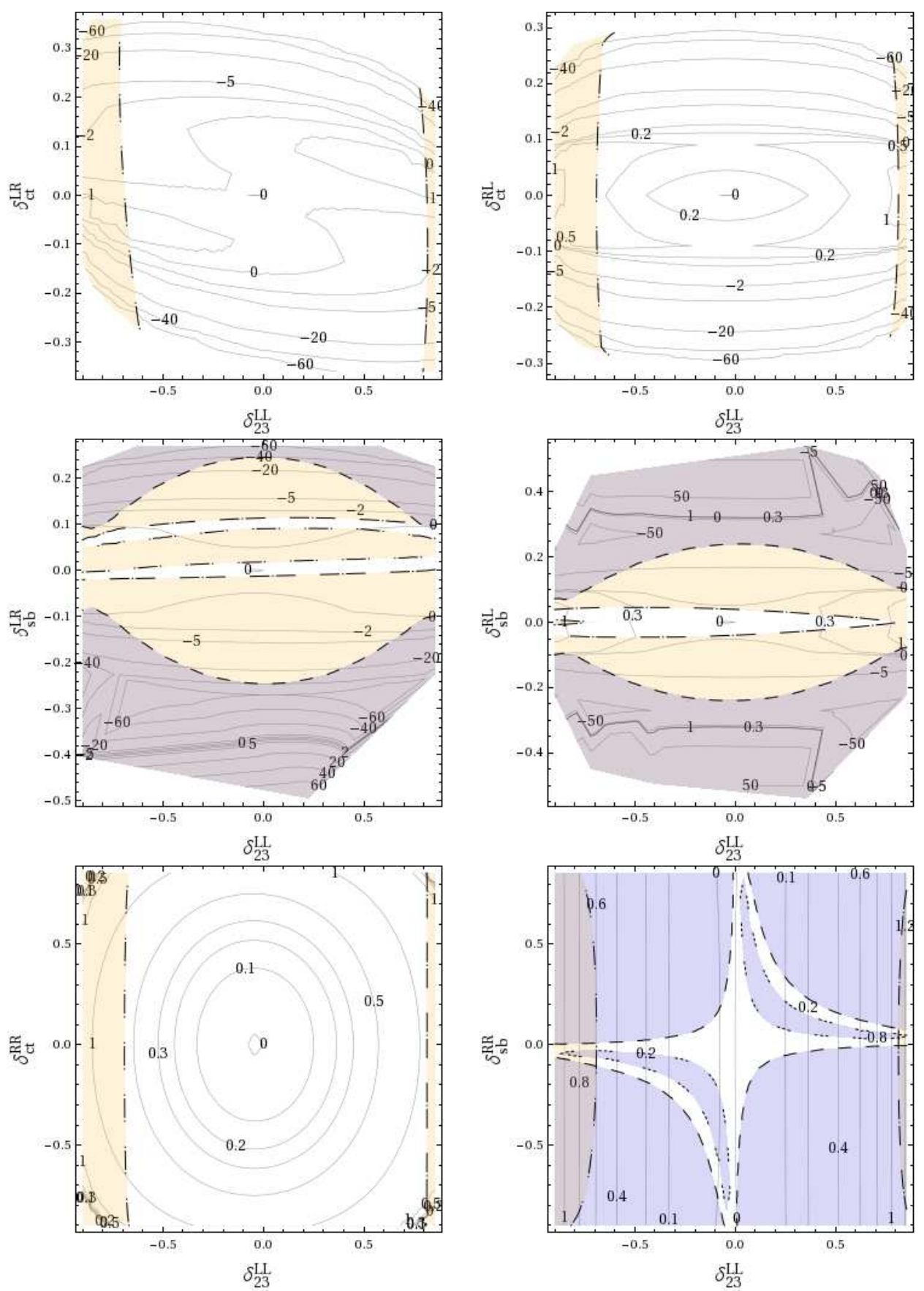

Figure 13. $\Delta m_{h}(\mathrm{GeV})$ contour lines from our two deltas analysis for VHeavyS. The color code for the allowed/disallowed areas by $B$ data is given in figure 8 .

For the evaluation of $\Delta M_{B_{s}}$ we have added the one-loop gluino boxes [44] which are known to be very relevant in the context of NMFV scenarios [45-47]. We have estimated the size of these corrections and compared them with the other relevant contributions from chargino boxes and double Higgs penguins for all values of $\tan \beta$ for the first time. And we have concluded that gluino boxes dominate for moderate and low $\tan \beta \leq 20$ 

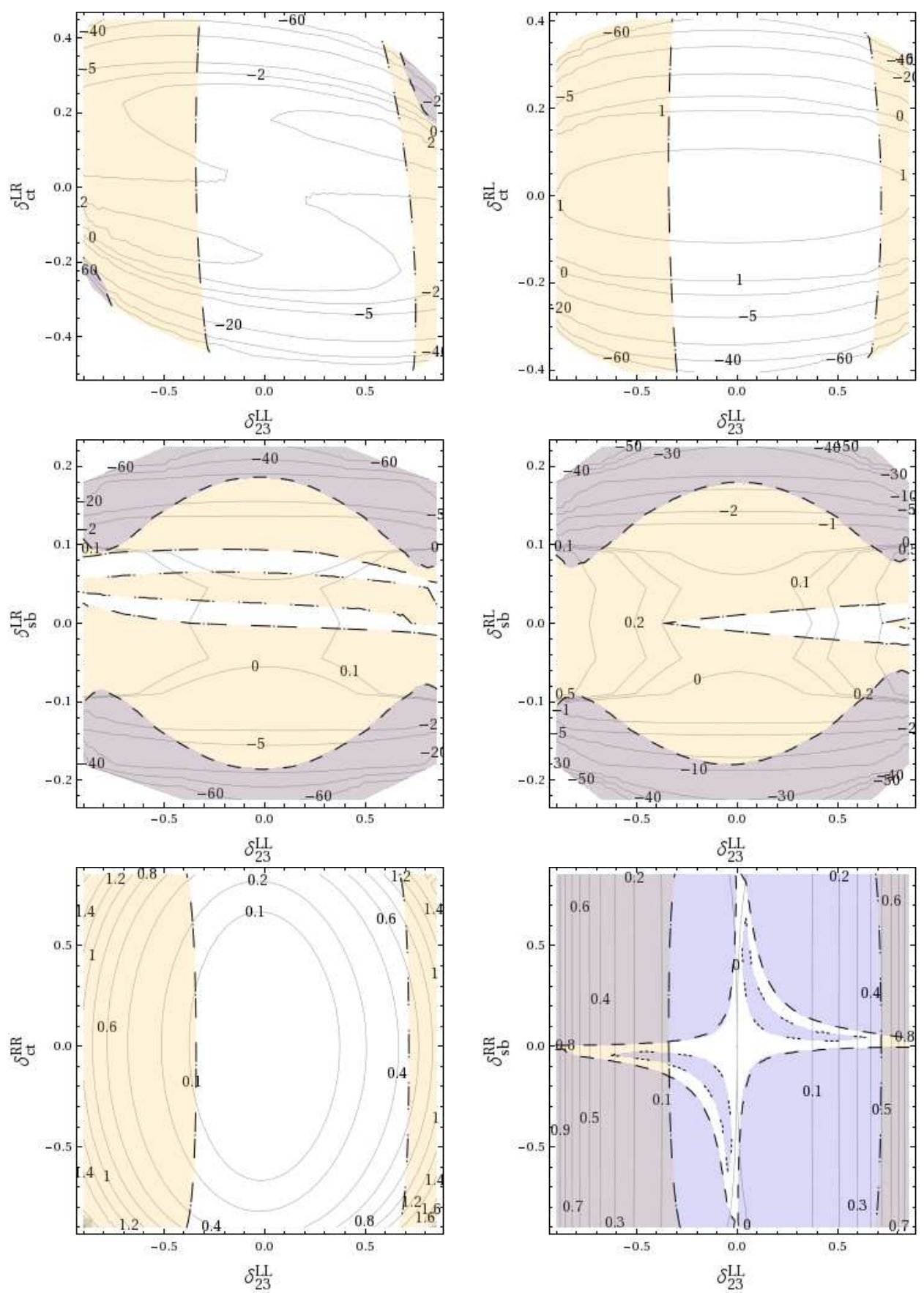

Figure 14. $\Delta m_{h}(\mathrm{GeV})$ contour lines from our two deltas analysis for HeavySLightH. The color code for the allowed/disallowed areas by $B$ data is given in figure 8 .

which is the interesting range for the present work. In the final part of the $B$ physics analysis, we have evaluated in one-dimensional scans which intervals for the $\delta_{i j}^{X Y}$ are still allowed in certain benchmark scenarios based on the SPS points.

In the second step we analyzed the one-loop contributions of NMFV to the MSSM Higgs boson masses, focusing on the parameter space still allowed by the experimental 
flavor constraints and by current limits from Higgs boson searches. Here two relevant $\delta_{i j}^{X Y}$ were varied simultaneously, thus enlarging the allowed range for these parameters. We found large corrections, mainly for the low $\tan \beta$ case, up to several tens of $\mathrm{GeV}$ for $m_{h}$ and somewhat smaller corrections for $m_{H}$ and $m_{H^{ \pm}}$. These corrections are specially relevant in the case of the light MSSM Higgs boson since they can be negative and up to two orders of magnitude larger than the anticipated LHC precision. Consequently, these corrections must be taken into account in any Higgs boson analysis in the NMFV MSSM framework. Conversely, in the case of a Higgs boson mass measurement these corrections might be used to set further constraints on $\delta_{i j}^{X Y}$. The present work clearly indicates that the flavor mixing parameters $\delta_{c t}^{L R}$ and $\delta_{c t}^{R L}$ are severely constrained by the present bounds on the lightest Higgs boson mass within the NMFV-MSSM scenarios.

\section{Acknowledgments}

We thank P. Paradisi and G. Isidori for kindly providing us the BPHYSICS subroutine and for helpful discussions. We thank T. Hahn for invaluable help with FeynHiggs, as well as FeynArts and FormCalc, and C. Pena for helpful discussions on $B$-physics. We are indebted to $\mathrm{P}$. Slavich for his valuable comments and corrections regarding $\mathrm{BR}\left(B \rightarrow X_{s} \gamma\right)$. We thank him for spotting the missing operators $O_{7,8}^{\prime}$ (eqs. (3.4) and (3.5)) in a preliminary version of this paper. S.H. thanks A. Crivellin, L. Hofer and U. Nierste for interesting discussions.

The work of S.H. was supported in part by CICYT (grant FPA 2007-66387), in part by CICYT (grant FPA 2010-22163-C02-01) and by the Spanish MICINN's Consolider-Ingenio 2010 Program under grant MultiDark CSD2009-00064. The work of M.H. and M.A.-C. was partially supported by CICYT (grant FPA2009-09017) and the Comunidad de Madrid project HEPHACOS, S2009/ESP-1473. The work of S.P. was supported by a Ramón y Cajal contract from MEC (Spain) (PDRYC-2006-000930) and partially by CICYT (grant FPA2009-09638), the Comunidad de Aragón project DCYT-DGA E24/2 and the Generalitat de Catalunya project 2009SGR502. The work is also supported in part by the European Community's Marie-Curie Research Training Network under contract MRTNCT-2006-035505 'Tools and Precision Calculations for Physics Discoveries at Colliders' and also by the Spanish Consolider-Ingenio 2010 Programme CPAN (CSD2007-00042).

\section{A Feynman rules}

We list the new Feynman rules of the NMFV scenario that are involved in the present computation. The corresponding couplings to the Higgs boson $H$ are obtained from the ones listened here for the lightest Higgs boson $h$ by replacing

$$
c_{\alpha} \rightarrow s_{\alpha} \quad ; \quad s_{\alpha} \rightarrow-c_{\alpha} \quad ; \quad s_{\alpha+\beta} \rightarrow-c_{\alpha+\beta} \quad ; \quad c_{2 \alpha} \rightarrow-c_{2 \alpha}
$$

The notation used for the formulas is the following: $s_{x}=\sin x ; c_{x}=\cos x ; s_{\mathrm{w}}=$ $\sin \theta_{W} ; c_{\mathrm{w}}=\cos \theta_{W}=\frac{M_{W}}{M_{Z}} ; t_{\beta}=\tan \beta$. 


\section{Couplings of two squarks and one/two Higgs bosons}

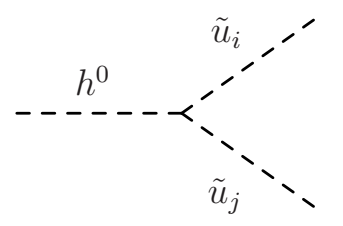

$$
\begin{aligned}
-\sum_{k, l=1}^{3} \frac{i e}{6 M_{W} c_{\mathrm{w}} s_{\mathrm{w}} s_{\beta}}\{ & R_{i, k}^{\tilde{u} *}\left\{\delta_{k l} R_{j, l}^{\tilde{u}}\left(6 c_{\alpha} c_{\mathrm{w}} m_{u_{k}}^{2}-M_{W} M_{Z} s_{\alpha+\beta} s_{\beta}\left(3-4 s_{\mathrm{w}}^{2}\right)\right)\right. \\
& \left.+3 c_{\mathrm{w}} R_{j, 3+l}^{\tilde{u}}\left(A_{k, l}^{u} c_{\alpha} m_{u_{k}}+\delta_{k l} m_{u_{k}} \mu^{*} s_{\alpha}\right)\right\} \\
& +R_{i, 3+k}^{\tilde{u}, *}\left\{\delta_{k l} R_{j, 3+l}^{\tilde{u}}\left(6 c_{\alpha} c_{\mathrm{w}} m_{u_{k}}^{2}-4 M_{W} M_{Z} s_{\alpha+\beta} s_{\beta} s_{\mathrm{w}}^{2}\right)\right. \\
& \left.\left.+3 c_{\mathrm{w}} R_{j, l}^{\tilde{u}}\left(A_{l, k}^{u *} c_{\alpha} m_{u_{l}}+\delta_{k l} m_{u_{k}} \mu s_{\alpha}\right)\right\}\right\}
\end{aligned}
$$

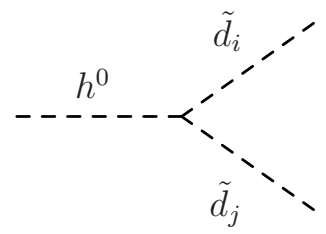

$\sum_{k, l=1}^{3} \frac{i e}{6 M_{W} c_{\mathrm{w}} s_{\mathrm{w}} c_{\beta}}\left\{R_{i, k}^{\tilde{d} *}\left\{\delta_{k l} R_{j, l}^{\tilde{d}}\left(6 s_{\alpha} c_{\mathrm{w}} m_{d_{k}}^{2}-M_{W} M_{Z} s_{\alpha+\beta} c_{\beta}\left(3-2 s_{\mathrm{w}}^{2}\right)\right)\right.\right.$

$$
\begin{aligned}
& \left.+3 c_{\mathrm{w}} R_{j, 3+l}^{\tilde{d}}\left(A_{k, l}^{d} s_{\alpha} m_{d_{k}}+\delta_{k l} m_{d_{k}} \mu^{*} c_{\alpha}\right)\right\} \\
& +R_{i, 3+k}^{\tilde{d} *}\left\{\delta_{k l} R_{j, 3+l}^{\tilde{d}}\left(6 s_{\alpha} c_{\mathrm{w}} m_{d_{k}}^{2}-2 M_{W} M_{Z} s_{\alpha+\beta} c_{\beta} s_{\mathrm{w}}^{2}\right)\right.
\end{aligned}
$$$$
\left.\left.+3 c_{\mathrm{w}} R_{j, l}^{\tilde{d}}\left(A_{l, k}^{d *} s_{\alpha} m_{d_{l}}+\delta_{k l} m_{d_{k}} \mu c_{\alpha}\right)\right\}\right\}
$$

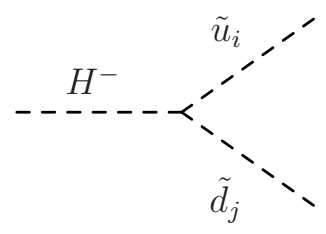

$\sum_{k, l=1}^{3} \frac{i e}{\sqrt{2} M_{W} s_{\mathrm{w}} t_{\beta}}\left\{R_{i, 3+k}^{\tilde{u} *}\left\{R_{j, l}^{\tilde{d}}\left(\sum_{n=1}^{3} A_{n, k}^{u *} m_{u_{n}} V_{\mathrm{CKM}}^{* n l}+m_{u_{k}} \mu V_{\mathrm{CKM}}^{* k l} t_{\beta}\right)\right.\right.$

$\left.+m_{d_{l}} m_{u_{k}} V_{\mathrm{CKM}}^{* k l} R_{j, 3+l}^{\tilde{d}}\left(1+t_{\beta}{ }^{2}\right)\right\}$

$+R_{i, k}^{\tilde{u} *}\left\{R_{j, 3+l}^{\tilde{d}} t_{\beta}\left(\sum_{n=1}^{3} A_{n, l}^{d} m_{d_{n}} V_{\mathrm{CKM}}^{* k n} t_{\beta}+m_{d_{l}} \mu^{*} V_{\mathrm{CKM}}^{* k l}\right)\right.$

$\left.\left.+V_{\mathrm{CKM}}^{* k l} R_{j, l}^{\tilde{d}}\left(m_{u_{k}}^{2}-t_{\beta}\left(M_{W}^{2} s_{2 \beta}-m_{d_{l}}^{2} t_{\beta}\right)\right)\right\}\right\}$

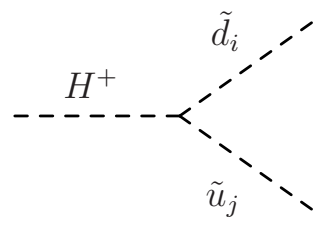

$\sum_{k, l=1}^{3} \frac{i e}{\sqrt{2} M_{W} s_{\mathrm{w}} t_{\beta}}\left\{R_{i, 3+l}^{\tilde{d} *}\left\{R_{j, k}^{\tilde{u}} t_{\beta}\left(\sum_{n=1}^{3} A_{n, l}^{d *} m_{d_{n}} V_{\mathrm{CKM}}^{k n} t_{\beta}+m_{d_{l}} \mu V_{\mathrm{CKM}}^{k l}\right)\right.\right.$

$\left.+m_{d_{l}} m_{u_{k}} V_{\mathrm{CKM}}^{k l} R_{j, 3+k}^{\tilde{u}}\left(1+t_{\beta}^{2}\right)\right\}$

$+R_{i, l}^{\tilde{d} *}\left\{R_{j, \tilde{u}+k}^{\tilde{u}}\left(\sum_{n=1}^{3} A_{n, k}^{u} m_{u_{n}} V_{\mathrm{CKM}}^{n l}+m_{u_{k}} \mu^{*} V_{\mathrm{CKM}}^{k l} t_{\beta}\right)\right.$

$\left.\left.+V_{\mathrm{CKM}}^{k l} R_{j, k}^{\tilde{u}}\left(m_{u_{k}}^{2}-t_{\beta}\left(M_{W}^{2} s_{2 \beta}-m_{d_{l}}^{2} t_{\beta}\right)\right)\right\}\right\}$

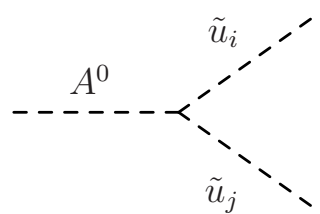

$-\sum_{k, l=1}^{3} \frac{e}{2 M_{W} s_{\mathrm{w}} t_{\beta}}\left\{R_{i, 3+k}^{\tilde{u} *} R_{j, l}^{\tilde{u}}\left(A_{l, k}^{u *} m_{u_{l}}+\delta_{k l} m_{u_{k}} \mu t_{\beta}\right)\right.$

$\left.-R_{i, k}^{\tilde{u} *} R_{j, 3+l}^{\tilde{u}}\left(A_{k, l}^{u} m_{u k}+\delta_{k l} m_{u_{k}} \mu^{*} t_{\beta}\right)\right\}$

$\tilde{d}_{i}$

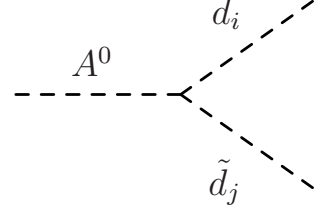

$$
\begin{array}{r}
-\sum_{k, l=1}^{3} \frac{e}{2 M_{W} s_{\mathrm{w}}}\left\{R_{i, 3+k}^{\tilde{d} *} R_{j, l}^{\tilde{d}}\left(A_{l, k}^{d *} m_{d l} t_{\beta}+\delta_{k l} m_{d_{k}} \mu\right)\right. \\
\left.-R_{i, k}^{\tilde{d} *} R_{j, 3+l}^{\tilde{d}}\left(A_{k, l}^{d} m_{d_{k}} t_{\beta}+\delta_{k l} m_{d_{k}} \mu^{*}\right)\right\}
\end{array}
$$

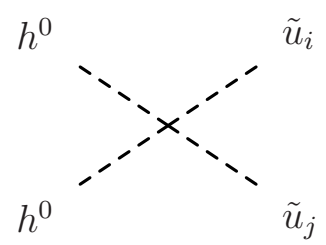

$$
\begin{aligned}
-\sum_{k=1}^{3} \frac{i e^{2}}{12 M_{W}^{2} c_{\mathrm{w}}^{2} s_{\mathrm{w}}^{2} s_{\beta}^{2}}\{ & R_{i, k}^{\tilde{u}} R_{j, k}^{\tilde{u} *}\left(6 c_{\alpha}^{2} c_{\mathrm{w}}^{2} m_{u_{k}}^{2}-c_{2 \alpha} M_{W}^{2} s_{\beta}^{2}\left(3-4 s_{\mathrm{w}}^{2}\right)\right) \\
& \left.+2 R_{i, 3+k}^{\tilde{u}} R_{j, 3+k}^{\tilde{u} *}\left(3 c_{\alpha}^{2} c_{\mathrm{w}}^{2} m_{u_{k}}^{2}-2 c_{2 \alpha} M_{W}^{2} s_{\beta}^{2} s_{\mathrm{w}}^{2}\right)\right\}
\end{aligned}
$$




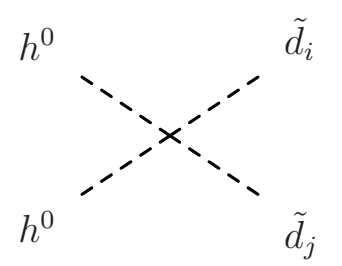

$$
\begin{aligned}
-\sum_{k=1}^{3} \frac{i e^{2}}{12 M_{W}^{2} c_{\mathrm{w}}^{2} s_{\mathrm{w}}^{2} c_{\beta}^{2}}\{ & R_{i, k}^{\tilde{d}} R_{j, k}^{\tilde{d} *}\left(6 s_{\alpha}^{2} c_{\mathrm{w}}^{2} m_{d_{k}}^{2}+c_{2 \alpha} M_{W}^{2} c_{\beta}^{2}\left(3-2 s_{\mathrm{w}}^{2}\right)\right) \\
& \left.+2 R_{i, 3+k}^{\tilde{d}} R_{j, 3+k}^{\tilde{d} *}\left(3 s_{\alpha}^{2} c_{\mathrm{w}}^{2} m_{d_{k}}^{2}+c_{2 \alpha} M_{W}^{2} c_{\beta}^{2} s_{\mathrm{w}}^{2}\right)\right\}
\end{aligned}
$$

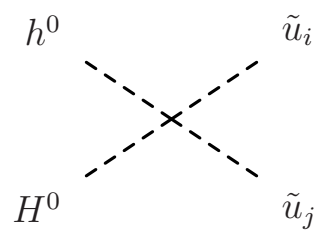

$$
\begin{aligned}
-\sum_{k=1}^{3} \frac{i e^{2} s_{2 \alpha}}{12 M_{W}^{2} c_{\mathrm{w}}^{2} s_{\mathrm{w}}^{2} s_{\beta}^{2}}\{ & R_{i, k}^{\tilde{u}} R_{j, k}^{\tilde{u} *}\left(3 c_{\mathrm{w}}^{2} m_{u_{k}}^{2}-M_{W}^{2} s_{\beta}^{2}\left(3-4 s_{\mathrm{w}}^{2}\right)\right) \\
& \left.+R_{i, 3+k}^{\tilde{u}} R_{j, 3+k}^{\tilde{u} *}\left(3 c_{\mathrm{w}}^{2} m_{u_{k}}^{2}-4 M_{W}^{2} s_{\beta}^{2} s_{\mathrm{w}}^{2}\right)\right\}
\end{aligned}
$$

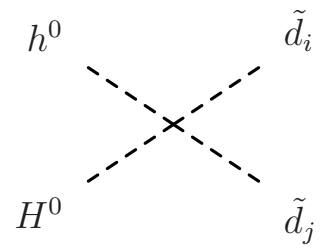

$$
\sum_{k=1}^{3} \frac{i e^{2} s_{2 \alpha}}{12 M_{W}^{2} c_{\mathrm{w}}^{2} s_{\mathrm{w}}^{2} c_{\beta}^{2}}\left\{R_{i, k}^{\tilde{d}} R_{j, k}^{\tilde{d} *}\left(3 c_{\mathrm{w}}^{2} m_{d_{k}}^{2}-M_{W}^{2} c_{\beta}^{2}\left(3-2 s_{\mathrm{w}}^{2}\right)\right)\right.
$$$$
\left.+R_{i, 3+k}^{\tilde{d}} R_{j, 3+k}^{\tilde{d} *}\left(3 c_{\mathrm{w}}^{2} m_{d_{k}}^{2}-2 M_{W}^{2} c_{\beta}^{2} s_{\mathrm{w}}^{2}\right)\right\}
$$

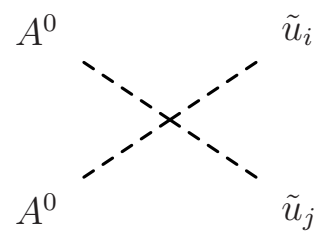

$-\sum_{k=1}^{3} \frac{i e^{2}}{12 M_{W}^{2} c_{\mathrm{w}}^{2} s_{\mathrm{w}}^{2} t_{\beta}^{2}}\left\{R_{i, k}^{\tilde{u}} R_{j, k}^{\tilde{u} *}\left(6 c_{\mathrm{w}}^{2} m_{u_{k}}^{2}-c_{2 \beta} M_{W}^{2} t_{\beta}^{2}\left(3-4 s_{\mathrm{w}}^{2}\right)\right)\right.$ $\left.+2 R_{i, 3+k}^{\tilde{u}} R_{j, 3+k}^{\tilde{u} *}\left(3 c_{\mathrm{w}}^{2} m_{u_{k}}^{2}-2 c_{2 \beta} M_{W}^{2} t_{\beta}^{2} s_{\mathrm{w}}^{2}\right)\right\}$

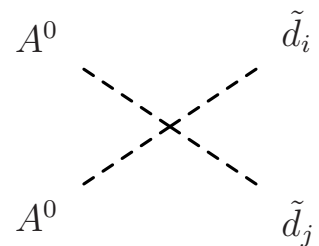

$$
-\sum_{k=1}^{3} \frac{i e^{2}}{12 M_{W}^{2} c_{\mathrm{w}}^{2} s_{\mathrm{w}}^{2}}\left\{R_{i, k}^{\tilde{d}} R_{j, k}^{\tilde{d} *}\left(6 c_{\mathrm{w}}^{2} m_{d_{k}}^{2} t_{\beta}^{2}+c_{2 \beta} M_{W}^{2}\left(3-2 s_{\mathrm{w}}^{2}\right)\right)\right.
$$$$
\left.+2 R_{i, 3+k}^{\tilde{d}} R_{j, 3+k}^{\tilde{d} *}\left(3 c_{\mathrm{w}}^{2} m_{d_{k}}^{2} t_{\beta}^{2}+c_{2 \beta} M_{W}^{2} s_{\mathrm{w}}^{2}\right)\right\}
$$<smiles>[3H]C([13CH3])([13CH3])[13CH3]</smiles>

$-\sum_{k, l=1}^{3} \frac{i e^{2}}{12 M_{W}^{2} s_{\mathrm{w}}^{2} c_{\mathrm{w}}^{2} t_{\beta}^{2}}\left\{R_{i, k}^{\tilde{u} *} R_{j, l}^{\tilde{u}} t_{\beta}^{2}\left(6 \sum_{n=1}^{3} m_{d_{n}}^{2} V_{\mathrm{CKM}}^{* k n} V_{\mathrm{CKM}}^{l n} c_{\mathrm{w}}^{2} t_{\beta}^{2}\right.\right.$ $\left.+c_{2 \beta} \delta_{k l} M_{W}^{2}\left(1+2 c_{\mathrm{w}}^{2}\right)\right)$ $\left.+2 \delta_{k l} R_{i, 3+k}^{\tilde{u} *} R_{j, 3+l}^{\tilde{u}}\left(3 c_{\mathrm{w}}^{2} m_{u_{k}}^{2}-2 c_{2 \beta} M_{W}^{2} s_{\mathrm{w}}^{2} t_{\beta}^{2}\right)\right\}$

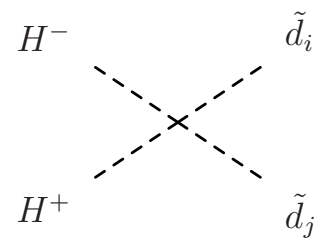

$-\sum_{k, l=1}^{3} \frac{i e^{2}}{12 M_{W}^{2} s_{\mathrm{w}}^{2} c_{\mathrm{w}}^{2} t_{\beta}^{2}}\left\{R_{i, k}^{\tilde{d} *} R_{j, l}^{\tilde{d}}\left(6 \sum_{n=1}^{3} m_{u_{n}}^{2} V_{\mathrm{CKM}}^{n k} V_{\mathrm{CKM}}^{* n l} c_{\mathrm{w}}^{2}\right.\right.$

$$
\begin{aligned}
& \left.+c_{2 \beta} \delta_{k l} M_{W}^{2} t_{\beta}^{2}\left(1-4 c_{\mathrm{w}}^{2}\right)\right) \\
& \left.+2 \delta_{k l} R_{i, 3+k}^{\tilde{d} *} R_{j, 3+l}^{\tilde{d}} t_{\beta}^{2}\left(3 c_{\mathrm{w}}^{2} t_{\beta}^{2} m_{d_{k}}^{2}+c_{2 \beta} M_{W}^{2} s_{\mathrm{w}}^{2}\right)\right\}
\end{aligned}
$$


3. Couplings of two squarks and one/two gauge bosons
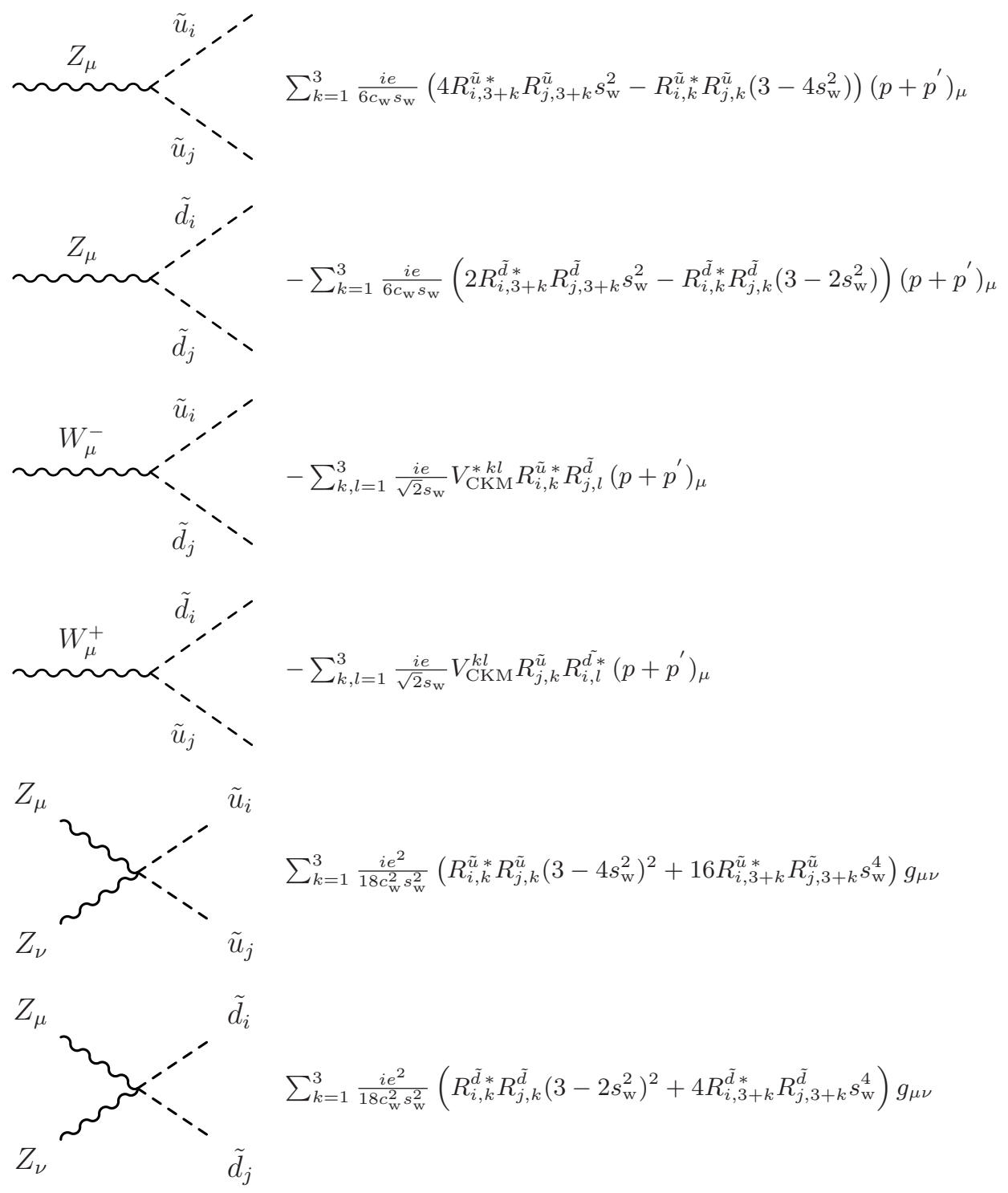

$\sum_{k=1}^{3} \frac{i e^{2}}{18 c_{\mathrm{W}}^{2} s_{\mathrm{w}}^{2}}\left(R_{i, k}^{\tilde{d} *} R_{j, k}^{\tilde{d}}\left(3-2 s_{\mathrm{w}}^{2}\right)^{2}+4 R_{i, 3+k}^{\tilde{d} *} R_{j, 3+k}^{\tilde{d}} s_{\mathrm{w}}^{4}\right) g_{\mu \nu}$

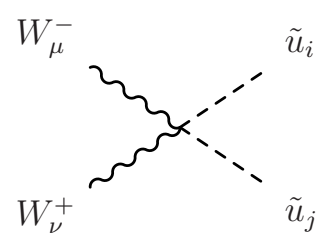

$\sum_{k=1}^{3} \frac{i e^{2}}{2 s_{\mathrm{w}}^{2}} R_{i, k}^{\tilde{u} *} R_{j, k}^{\tilde{u}} g_{\mu \nu}$

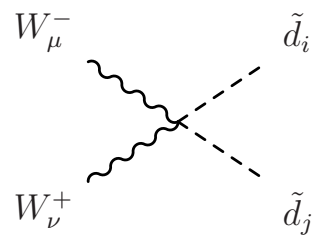

$\sum_{k=1}^{3} \frac{i e^{2}}{2 s_{\mathrm{w}}^{2}} R_{i, k}^{\tilde{d} *} R_{j, k}^{\tilde{d}} g_{\mu \nu}$ 

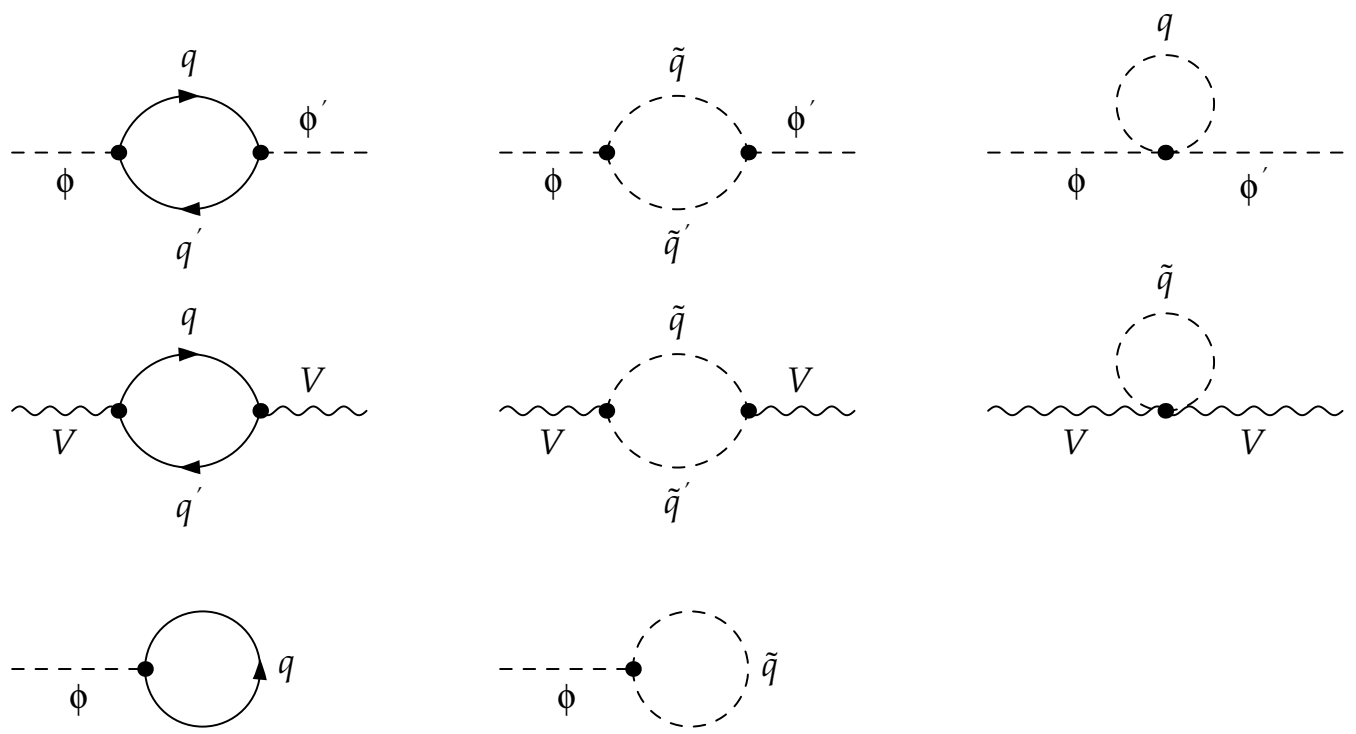

Figure 15. Different topologies for $\Sigma_{\phi \phi^{\prime}}, \Sigma_{V V}, T_{\phi}$

\section{B Tadpoles and self-energies}

All the following Feynman diagrams have been calculated using FeynArts 3.5 [50-52] and FormCalc 6.0 [53]. The notation used here is the same as in appendix A. Furthermore we use the functions [92]

$$
\begin{aligned}
\frac{i}{16 \pi} A_{0}\left[m^{2}\right] & \equiv \int \frac{\mu^{4-D} d^{D} k}{(2 \pi)^{D}} \frac{1}{k^{2}-m^{2}} \\
\frac{i}{16 \pi} B_{0}\left[p^{2}, m_{1}^{2}, m_{2}^{2}\right] & \equiv \int \frac{\mu^{4-D} d^{D} k}{(2 \pi)^{D}} \frac{1}{\left[k^{2}-m_{1}^{2}\right]\left[(k+p)^{2}-m_{2}^{2}\right]} \\
\frac{i}{16 \pi} p^{2} B_{1}\left[p^{2}, m_{1}^{2}, m_{2}^{2}\right] & \equiv \int \frac{\mu^{4-D} d^{D} k}{(2 \pi)^{D}} \frac{p k}{\left[k^{2}-m_{1}^{2}\right]\left[(k+p)^{2}-m_{2}^{2}\right]}
\end{aligned}
$$

The generic diagrams have been ordered according to its topologies, and the particles involved in the internal loops (quarks $q$ or squarks $\tilde{q}$ ). The diagrams can be found in figure 15. The complete self-energy can be expressed as a sum of three parts:

$$
\Sigma_{\phi \phi^{\prime}}=\Sigma_{\phi \phi^{\prime}}^{2 q}+\Sigma_{\phi \phi^{\prime}}^{2 \tilde{q}}+\Sigma_{\phi \phi^{\prime}}^{1 \tilde{q}} \quad \Sigma_{V V}=\Sigma_{V V}^{2 q}+\Sigma_{V V}^{2 \tilde{q}}+\Sigma_{V V}^{1 \tilde{q}} \quad T_{\phi}=T_{\phi}^{q}+T_{\phi}^{\tilde{q}}
$$

where $\phi, \phi^{\prime}=h, H, A, H^{ \pm}$and $V=W, Z$. All the self-energies $\Sigma$ correspond to $\Sigma\left(p^{2}\right)$. The self-energies for $H$ are obtained by the replacements of eq. (A.1) on the results of $h$ :

- $h$

$$
\begin{aligned}
\Sigma_{h h}^{2 q}= & -\sum_{i=1}^{3} \frac{3 \alpha c_{\alpha}^{2} m_{u_{i}}^{2}}{4 \pi M_{W}^{2} s_{\beta}^{2} s_{W}^{2}}\left\{A_{0}\left[m_{u_{i}}^{2}\right]+p^{2} B_{1}\left[p^{2}, m_{u_{i}}^{2}, m_{u_{i}}^{2}\right]+2 m_{u_{i}}^{2} B_{0}\left[p^{2}, m_{u_{i}}^{2}, m_{u_{i}}^{2}\right]\right\} \\
& -\sum_{i=1}^{3} \frac{3 \alpha s_{\alpha}^{2} m_{d_{i}}^{2}}{4 \pi M_{W}^{2} c_{\beta}^{2} s_{W}^{2}}\left\{A_{0}\left[m_{d_{i}}^{2}\right]+p^{2} B_{1}\left[p^{2}, m_{d_{i}}^{2}, m_{d_{i}}^{2}\right]+2 m_{d_{i}}^{2} B_{0}\left[p^{2}, m_{d_{i}}^{2}, m_{d_{i}}^{2}\right]\right\}
\end{aligned}
$$




$$
\begin{aligned}
& \Sigma_{h h}^{2 \tilde{q}}=\sum_{m, n}^{6} \sum_{i, j, k, l}^{3} \frac{1}{48 c_{W}^{2} M_{W}^{2} \pi s_{\beta}^{2} s_{W}^{2}} \alpha B_{0}\left[p^{2}, m_{\tilde{u}_{m}}^{2}, m_{\tilde{u}_{n}}^{2}\right] \\
& \times\left\{\delta_{i, j}\left(M_{W} m_{Z} s_{\alpha+\beta} s_{\beta}\left(-3+4 s_{W}^{2}\right)+6 c_{\alpha} c_{W} m_{u_{i}}^{2}\right) R_{n, j}^{\tilde{u}} R_{m, i}^{\tilde{u} *}\right. \\
& +3 c_{W}\left(c_{\alpha} A_{i, j}^{u}+\mu^{*} s_{\alpha} \delta_{i, j}\right) m_{u_{i}} R_{n, 3+j}^{\tilde{u}} R_{m, i}^{\tilde{u} *} \\
& +\left(3 c_{\alpha} c_{W} A_{j, i}^{u *} m_{u_{j}} R_{n, j}^{\tilde{u}}+3 c_{W} \mu s_{\alpha} \delta_{i, j} m_{u_{i}} R_{n, j}^{\tilde{u}}\right) R_{m, 3+i}^{\tilde{u} *} \\
& \left.+2 \delta_{i, j}\left(-2 M_{W} m_{Z} s_{\alpha+\beta} s_{\beta} s_{W}^{2}+3 c_{\alpha} c_{W} m_{u_{i}}^{2}\right) R_{n, 3+j}^{\tilde{u}} R_{m, 3+i}^{\tilde{u} *}\right\} \\
& \times\left\{\delta_{k, l}\left(M_{W} m_{Z} s_{\alpha+\beta} s_{\beta}\left(-3+4 s_{W}^{2}\right)+6 c_{\alpha} c_{W} m_{u_{k}}^{2}\right) R_{m, l}^{\tilde{u}} R_{n, k}^{\tilde{u} *}\right. \\
& +3 c_{W}\left(c_{\alpha} A_{k, l}^{u}+\mu^{*} s_{\alpha} \delta_{k, l}\right) m_{u_{k}} R_{m, 3+l}^{\tilde{u}} R_{n, k}^{\tilde{u} *} \\
& +\left(3 c_{\alpha} c_{W} A_{l, k}^{u *} m_{u_{l}} R_{m, l}^{\tilde{u}}+3 c_{W} \mu s_{\alpha} \delta_{k, l} m_{u_{k}} R_{m, l}^{\tilde{u}}\right) R_{n, 3+k}^{\tilde{u} *} \\
& \left.+2 \delta_{k, l}\left(-2 M_{W} m_{Z} s_{\alpha+\beta} s_{\beta} s_{W}^{2}+3 c_{\alpha} c_{W} m_{u_{k}}^{2}\right) R_{m, 3+l}^{\tilde{u}} R_{n, 3+k}^{\tilde{u} *}\right\} \\
& +\sum_{m, n}^{6} \sum_{i, j, k, l}^{3} \frac{1}{48 c_{W}^{2} M_{W}^{2} \pi c_{\beta}^{2} s_{W}^{2}} \alpha B_{0}\left[p^{2}, m_{\tilde{d}_{m}}^{2}, m_{\tilde{d}_{n}}^{2}\right] \\
& \times\left\{\delta_{i, j}\left(M_{W} m_{Z} s_{\alpha+\beta} c_{\beta}\left(-3+2 s_{W}^{2}\right)+6 s_{\alpha} c_{W} m_{d_{i}}^{2}\right) R_{n, j}^{\tilde{d}} R_{m, i}^{\tilde{d} *}\right. \\
& +3 c_{W}\left(s_{\alpha} A_{i, j}^{d}+\mu^{*} c_{\alpha} \delta_{i, j}\right) m_{d_{i}} R_{n, 3+j}^{\tilde{d}} R_{m, i}^{\tilde{d} *} \\
& +\left(3 s_{\alpha} c_{W} A_{j, i}^{d *} m_{d_{j}} R_{n, j}^{\tilde{d}}+3 c_{W} \mu c_{\alpha} \delta_{i, j} m_{d_{i}} R_{n, j}^{\tilde{d}}\right) R_{m, 3+i}^{\tilde{d} *} \\
& \left.-2 \delta_{i, j}\left(M_{W} m_{Z} s_{\alpha+\beta} c_{\beta} s_{W}^{2}-3 s_{\alpha} c_{W} m_{d_{i}}^{2}\right) R_{n, 3+j}^{\tilde{d}} R_{m, 3+i}^{\tilde{d} *}\right\} \\
& \times\left\{\left(\delta_{k, l}\left(M_{W} m_{Z} s_{\alpha+\beta} c_{\beta}\left(-3+2 s_{W}^{2}\right)+6 s_{\alpha} c_{W} m_{d_{k}}^{2}\right) R_{m, l}^{\tilde{d}} R_{n, k}^{\tilde{d} *}\right)\right. \\
& +3 c_{W}\left(s_{\alpha} A_{k, l}^{d}+\mu^{*} c_{\alpha} \delta_{k, l}\right) m_{d_{k}} R_{m, 3+l}^{\tilde{d}} R_{n, k}^{\tilde{d} *} \\
& +\left(3 s_{\alpha} c_{W} A_{l, k}^{d *} m_{d_{l}} R_{m, l}^{\tilde{d}}+3 c_{W} \mu c_{\alpha} \delta_{k, l} m_{d_{k}} R_{m, l}^{\tilde{d}}\right) R_{n, 3+k}^{\tilde{d} *} \\
& \left.-2 \delta_{k, l}\left(M_{W} m_{Z} s_{\alpha+\beta} c_{\beta} s_{W}^{2}-3 s_{\alpha} c_{W} m_{d_{k}}^{2}\right) R_{m, 3+l}^{\tilde{d}} R_{n, 3+k}^{\tilde{d} *}\right\} \\
& \Sigma_{h h}^{1 \tilde{q}}=\sum_{l=1}^{6} \sum_{i=1}^{3} \frac{1}{16 c_{W}^{2} M_{W}^{2} \pi s_{\beta}^{2} s_{W}^{2}} \alpha A_{0}\left[m_{\tilde{u}_{l}}^{2}\right]\left\{R_{l, i}^{\tilde{u}} R_{l, i}^{\tilde{u} *}\left(c_{2 \alpha} m_{w}^{2} s_{\beta}^{2}\left(-3+4 s_{W}^{2}\right)+6 c_{\alpha}^{2} c_{W}^{2} m_{u_{i}}^{2}\right)\right. \\
& \left.+2 R_{l, 3+i}^{\tilde{u}} R_{l, 3+i}^{\tilde{u} *}\left(-2 c_{2 \alpha} M_{W}^{2} s_{\beta}^{2} s_{W}^{2}+3 c_{\alpha}^{2} c_{W}^{2} m_{u_{i}}^{2}\right)\right\}
\end{aligned}
$$




$$
\begin{aligned}
& -\sum_{l=1}^{6} \sum_{i=1}^{3} \frac{1}{16 c_{W}^{2} M_{W}^{2} \pi c_{\beta}^{2} s_{W}^{2}} \alpha A_{0}\left[m_{\tilde{d}_{l}}^{2}\right]\left\{R_{l, i}^{\tilde{d}} R_{l, i}^{\tilde{d} *}\left(c_{2 \alpha} m_{w}^{2} c_{\beta}^{2}\left(-3+2 s_{W}^{2}\right)-6 s_{\alpha}^{2} c_{W}^{2} m_{d_{i}}^{2}\right)\right. \\
& \left.-2 R_{l, 3+i}^{\tilde{d}} R_{l, 3+i}^{\tilde{d} *}\left(c_{2 \alpha} M_{W}^{2} c_{\beta}^{2} s_{W}^{2}+3 s_{\alpha}^{2} c_{W}^{2} m_{d_{i}}^{2}\right)\right\}
\end{aligned}
$$

- $h H$

$$
\begin{aligned}
& \Sigma_{h H}^{2 q}=-\sum_{i=1}^{3} \frac{3 \alpha c_{\alpha} s_{\alpha} m_{u_{i}}^{2}}{4 \pi M_{W}^{2} s_{\beta}^{2} s_{W}^{2}}\left\{A_{0}\left[m_{u_{i}}^{2}\right]+p^{2} B_{1}\left[p^{2}, m_{u_{i}}^{2}, m_{u_{i}}^{2}\right]+2 m_{u_{i}}^{2} B_{0}\left[p^{2}, m_{u_{i}}^{2}, m_{u_{i}}^{2}\right]\right\} \\
& +\sum_{i=1}^{3} \frac{3 \alpha c_{\alpha} s_{\alpha} m_{d_{i}}^{2}}{4 \pi M_{W}^{2} c_{\beta}^{2} s_{W}^{2}}\left\{A_{0}\left[m_{d_{i}}^{2}\right]+p^{2} B_{1}\left[p^{2}, m_{d_{i}}^{2}, m_{d_{i}}^{2}\right]+2 m_{d_{i}}^{2} B_{0}\left[p^{2}, m_{d_{i}}^{2}, m_{d_{i}}^{2}\right]\right\} \\
& \Sigma_{h H}^{2 \tilde{q}}=\sum_{m, n}^{6} \sum_{i, j, k, l}^{3} \frac{1}{48 c_{W}^{2} M_{W}^{2} \pi s_{\beta}^{2} s_{W}^{2}} \alpha B_{0}\left[p^{2}, m_{\tilde{u}_{m}}^{2}, m_{\tilde{u}_{n}}^{2}\right] \\
& \times\left\{\delta_{i, j}\left(M_{W} m_{Z} s_{\alpha+\beta} s_{\beta}\left(-3+4 s_{W}^{2}\right)+6 c_{\alpha} c_{W} m_{u_{i}}^{2}\right) R_{n, j}^{\tilde{u}} R_{m, i}^{\tilde{u} *}\right. \\
& +3 c_{W}\left(c_{\alpha} A_{i, j}^{u}+\mu^{*} s_{\alpha} \delta_{i, j}\right) m_{u_{i}} R_{n, 3+j}^{\tilde{u}} R_{m, i}^{\tilde{u} *} \\
& +\left(3 c_{\alpha} c_{W} A_{j, i}^{u *} m_{u_{j}} R_{n, j}^{\tilde{u}}+3 c_{W} \mu s_{\alpha} \delta_{i, j} m_{u_{i}} R_{n, j}^{\tilde{u}}\right) R_{m, 3+i}^{\tilde{u} *} \\
& \left.+2 \delta_{i, j}\left(-2 M_{W} m_{Z} s_{\alpha+\beta} s_{\beta} s_{W}^{2}+3 c_{\alpha} c_{W} m_{u_{i}}^{2}\right) R_{n, 3+j}^{\tilde{u}} R_{m, 3+i}^{\tilde{u} *}\right\} \\
& \times\left\{\delta_{k, l}\left(M_{W} m_{Z} c_{\alpha+\beta} s_{\beta}\left(3-4 s_{W}^{2}\right)+6 s_{\alpha} c_{W} m_{u_{k}}^{2}\right) R_{m, l}^{\tilde{u}} R_{n, k}^{\tilde{u} *}\right. \\
& +3 c_{W}\left(s_{\alpha} A_{k, l}^{u}-\mu^{*} c_{\alpha} \delta_{k, l}\right) m_{u_{k}} R_{m, 3+l}^{\tilde{u}} R_{n, k}^{\tilde{u} *} \\
& +\left(3 s_{\alpha} c_{W} A_{l, k}^{u *} m_{u_{l}} R_{m, l}^{\tilde{u}}-3 c_{W} \mu c_{\alpha} \delta_{k, l} m_{u_{k}} R_{m, l}^{\tilde{u}}\right) R_{n, 3+k}^{\tilde{u} *} \\
& \left.+2 \delta_{k, l}\left(2 M_{W} m_{Z} c_{\alpha+\beta} s_{\beta} s_{W}^{2}+3 s_{\alpha} c_{W} m_{u_{k}}^{2}\right) R_{m, 3+l}^{\tilde{u}} R_{n, 3+k}^{\tilde{u} *}\right\} \\
& -\sum_{m, n}^{6} \sum_{i, j, k, l}^{3} \frac{1}{48 c_{W}^{2} M_{W}^{2} \pi c_{\beta}^{2} s_{W}^{2}} \alpha B_{0}\left[p^{2}, m_{\tilde{d}_{m}}^{2}, m_{\tilde{d}_{n}}^{2}\right] \\
& \times\left\{\delta_{i, j}\left(M_{W} m_{Z} s_{\alpha+\beta} c_{\beta}\left(-3+2 s_{W}^{2}\right)+6 s_{\alpha} c_{W} m_{d_{i}}^{2}\right) R_{n, j}^{\tilde{d}} R_{m, i}^{\tilde{d} *}\right. \\
& +3 c_{W}\left(s_{\alpha} A_{i, j}^{d}+\mu^{*} c_{\alpha} \delta_{i, j}\right) m_{d_{i}} R_{n, 3+j}^{\tilde{d}} R_{m, i}^{\tilde{d} *} \\
& +\left(3 s_{\alpha} c_{W} A_{j, i}^{d *} m_{d_{j}} R_{n, j}^{\tilde{d}}+3 c_{W} \mu c_{\alpha} \delta_{i, j} m_{d_{i}} R_{n, j}^{\tilde{d}}\right) R_{m, 3+i}^{\tilde{d} *} \\
& \left.-2 \delta_{i, j}\left(M_{W} m_{Z} s_{\alpha+\beta} c_{\beta} s_{W}^{2}-3 s_{\alpha} c_{W} m_{d_{i}}^{2}\right) R_{n, 3+j}^{\tilde{d}} R_{m, 3+i}^{\tilde{d} *}\right\} \\
& \times\left\{\delta_{k, l}\left(M_{W} m_{Z} c_{\alpha+\beta} c_{\beta}\left(-3+2 s_{W}^{2}\right)+6 c_{\alpha} c_{W} m_{d_{k}}^{2}\right) R_{m, l}^{\tilde{d}} R_{n, k}^{\tilde{d} *}\right.
\end{aligned}
$$




$$
\begin{aligned}
& +3 c_{W}\left(c_{\alpha} A_{k, l}^{d}-\mu^{*} s_{\alpha} \delta_{k, l}\right) m_{d_{k}} R_{m, 3+l}^{\tilde{d}} R_{n, k}^{\tilde{d} *} \\
& +\left(3 c_{\alpha} c_{W} A_{l, k}^{d *} m_{d_{l}} R_{m, l}^{\tilde{d}}-3 c_{W} \mu s_{\alpha} \delta_{k, l} m_{d_{k}} R_{m, l}^{\tilde{d}}\right) R_{n, 3+k}^{\tilde{d} *} \\
& \left.-2 \delta_{k, l}\left(2 M_{W} m_{Z} c_{\alpha+\beta} c_{\beta} s_{W}^{2}-3 c_{\alpha} c_{W} m_{d_{k}}^{2}\right) R_{m, 3+l}^{\tilde{d}} R_{n, 3+k}^{\tilde{d} *}\right\} \\
\Sigma_{h H}^{1 \tilde{q}}= & \sum_{l=1}^{6} \sum_{i=1}^{3} \frac{1}{16 c_{W}^{2} M_{W}^{2} \pi s_{\beta}^{2} s_{W}^{2}} \alpha A_{0}\left[m_{\tilde{u}_{l}}^{2}\right]\left\{R_{l, i}^{\tilde{u}} R_{l, i}^{\tilde{u} *}\left(s_{2 \alpha} m_{w}^{2} s_{\beta}^{2}\left(-3+4 s_{W}^{2}\right)+3 s_{2 \alpha} c_{W}^{2} m_{u_{i}}^{2}\right)\right. \\
& \left.+R_{l, 3+i}^{\tilde{u}} R_{l, 3+i}^{\tilde{u} *}\left(-4 s_{2 \alpha} M_{W}^{2} s_{\beta}^{2} s_{W}^{2}+3 s_{2 \alpha} c_{W}^{2} m_{u_{i}}^{2}\right)\right\} \\
& -\sum_{l=1}^{6} \sum_{i=1}^{3} \frac{1}{16 c_{W}^{2} M_{W}^{2} \pi c_{\beta}^{2} s_{W}^{2}} \alpha A_{0}\left[m_{\tilde{d}_{l}}^{2}\right]\left\{R_{l, i}^{\tilde{d}} R_{l, i}^{\tilde{d} *}\left(s_{2 \alpha} m_{w}^{2} c_{\beta}^{2}\left(-3+4 s_{W}^{2}\right)+3 s_{2 \alpha} c_{W}^{2} m_{d_{i}}^{2}\right)\right. \\
& \left.+R_{l, 3+i}^{\tilde{d}} R_{l, 3+i}^{\tilde{d} *}\left(-2 s_{2 \alpha} M_{W}^{2} c_{\beta}^{2} s_{W}^{2}+3 s_{2 \alpha} c_{W}^{2} m_{d_{i}}^{2}\right)\right\}
\end{aligned}
$$

- $A$

$$
\begin{aligned}
\Sigma_{A A}^{2 q}= & -\sum_{i=1}^{3} \frac{3 \alpha m_{u_{i}}^{2}}{4 \pi M_{W}^{2} t_{\beta}^{2} s_{W}^{2}}\left\{A_{0}\left[m_{u_{i}}^{2}\right]+p^{2} B_{1}\left[p^{2}, m_{u_{i}}^{2}, m_{u_{i}}^{2}\right]\right\} \\
& -\sum_{i=1}^{3} \frac{3 \alpha t_{\beta}^{2} m_{d_{i}}^{2}}{4 \pi M_{W}^{2} s_{W}^{2}}\left\{A_{0}\left[m_{d_{i}}^{2}\right]+p^{2} B_{1}\left[p^{2}, m_{d_{i}}^{2}, m_{d_{i}}^{2}\right]\right\} \\
\Sigma_{A A}^{2 \tilde{q}}= & -\sum_{m, n}^{6} \sum_{i, j, k, l}^{3} \frac{3}{16 M_{W}^{2} \pi t_{\beta}^{2} s_{W}^{2}} \alpha B_{0}\left[p^{2}, m_{\tilde{u}_{m}}^{2}, m_{\tilde{u}_{n}}^{2}\right] \\
& \times\left\{\left(-A_{i, j}^{u}-\mu^{*} t_{\beta} \delta_{i, j}\right) m_{u_{i}} R_{n, 3+j}^{\tilde{u}} R_{m, i}^{\tilde{u} *}+\left(A_{j, i}^{u *} m_{u_{j}}+\mu t_{\beta} \delta_{i, j} m_{u_{i}}\right) R_{n, j}^{\tilde{u}} R_{m, 3+i}^{\tilde{u} *}\right\} \\
& \times\left\{\left(-A_{k, l}^{u}-\mu^{*} t_{\beta} \delta_{k, l}\right) m_{u_{k}} R_{m, 3+l}^{\tilde{u}} R_{n, k}^{\tilde{u} *}+\left(A_{l, k}^{u *} m_{u_{l}}+\mu t_{\beta} \delta_{k, l} m_{u_{k}}\right) R_{m, l}^{\tilde{u}} R_{n, 3+k}^{\tilde{u} *}\right\} \\
& -\sum_{m, n}^{6} \sum_{i, j, k, l}^{3} \frac{3}{16 M_{W}^{2} \pi s_{W}^{2}} \alpha B_{0}\left[p^{2}, m_{\tilde{d}_{m}}^{2}, m_{\tilde{d}_{n}}^{2}\right] \\
& \times\left\{\left(-t_{\beta} A_{i, j}^{d}-\mu^{*} \delta_{i, j}\right) m_{d_{i}} R_{n, 3+j}^{\tilde{d}} R_{m, i}^{\tilde{d} *}+\left(t_{\beta} A_{j, i}^{d *} m_{d_{j}}+\mu \delta_{i, j} m_{d_{i}}\right) R_{n, j}^{\tilde{d}} R_{m, 3+i}^{\tilde{d} *}\right\} \\
& \times\left\{\left(-t_{\beta} A_{k, l}^{d}-\mu^{*} \delta_{k, l}\right) m_{d_{k}} R_{m, 3+l}^{\tilde{d}} R_{n, k}^{\tilde{d} *}+\left(t_{\beta} A_{l, k}^{d *} m_{d_{l}}+\mu \delta_{k, l} m_{d_{k}}\right) R_{m, l}^{\tilde{d}} R_{n, 3+k}^{\tilde{d} *}\right\} \\
\Sigma_{A A}^{1 \tilde{q}}= & \sum_{l=1} \sum_{i=1}^{3} \frac{1}{16 c_{W}^{2} M_{W}^{2} \pi t_{\beta}^{2} s_{W}^{2}} \alpha A_{0}\left[m_{\tilde{u}_{l}}\right]\left\{R_{l, i}^{\tilde{u}} R_{l, i}^{\tilde{u} *}\left(c_{2 \beta} m_{w}^{2} t_{\beta}^{2}\left(-3+4 s_{W}^{2}\right)+6 c_{W}^{2} m_{u_{i}}^{2}\right)\right. \\
& \left.+2 R_{l, 3+i}^{\tilde{u}} R_{l, 3+i}^{\tilde{u} *}\left(-2 c_{2 \beta} M_{W}^{2} t_{\beta}^{2} s_{W}^{2}+3 c_{W}^{2} m_{u_{i}}^{2}\right)\right\} \\
& +\sum_{l=1} \sum_{i=1}^{3} \frac{1}{16 c_{W}^{2} M_{W}^{2} \pi s_{W}^{2}} \alpha A_{0}\left[m_{\tilde{d}_{l}}^{2}\right]\left\{R_{l, i}^{\tilde{d}} R_{l, i}^{\tilde{d} *}\left(c_{2 \beta} m_{w}^{2}\left(3-2 s_{W}^{2}\right)+6 c_{W}^{2} t_{\beta}^{2} m_{d_{i}}^{2}\right)\right. \\
& \left.+2 R_{l, 3+i}^{\tilde{d}} R_{l, 3+i}^{\tilde{d} *}\left(c_{2 \beta} M_{W}^{2} s_{W}^{2}+3 c_{W}^{2} t_{\beta}^{2} m_{d_{i}}^{2}\right)\right\}
\end{aligned}
$$


- $H^{ \pm}$

$$
\begin{aligned}
& \Sigma_{H^{-} H^{+}}^{2 q}=-\sum_{i=1}^{3} \sum_{j=1}^{3} \frac{3 \alpha}{4 \pi M_{W}^{2} s_{W}^{2}}\left\{m_{u_{i}}^{2}\left(2 m_{d_{j}}^{2}+m_{u_{i}}^{2} / t_{\beta}^{2}+m_{d_{j}}^{2} t_{\beta}^{2}\right) V_{\mathrm{CKM}}^{i, j} V_{\mathrm{CKM}}^{i, j *} B_{0}\left[p^{2}, m_{d_{j}}^{2}, m_{u_{i}}^{2}\right]\right. \\
& +\left(m_{u_{i}}^{2} / t_{\beta}^{2}+m_{d_{j}}^{2} t_{\beta}^{2}\right) V_{\mathrm{CKM}}^{i, j} V_{\mathrm{CKM}}^{i, j *} p^{2} B_{1}\left[p^{2}, m_{u_{i}}^{2}, m_{d_{j}}^{2}\right] \\
& \left.+A_{0}\left[m_{d_{j}}^{2}\right]\left(m_{u_{i}}^{2} V_{\mathrm{CKM}}^{i, j} V_{\mathrm{CKM}}^{i, j *} / t_{\beta}^{2}+m_{d_{j}}^{2} V_{\mathrm{CKM}}^{i, j} V_{\mathrm{CKM}}^{i, j *} t_{\beta}^{2}\right)\right\} \\
& \Sigma_{H^{-} H^{+}}^{2 \tilde{q}}=-\sum_{m, n}^{6} \sum_{i, j, k, l}^{3} \frac{3}{8 M_{W}^{2} \pi t_{\beta}^{2} s_{W}^{2}} \alpha B_{0}\left[p^{2}, m_{\tilde{u}_{m}}^{2}, m_{\tilde{u}_{n}}^{2}\right] \\
& \times\left\{\sum _ { p , q } ^ { 3 } \left[\left(t_{\beta}^{2} A_{p, i}^{d} V_{\mathrm{CKM}}^{k, p *} m_{d_{p}} R_{n, 3+i}^{\tilde{d}} R_{m, k}^{\tilde{u} *}+A_{p, k}^{u *} V_{\mathrm{CKM}}^{p, i *} m_{u_{p}} R_{n, i}^{\tilde{d}} R_{m, 3+k}^{\tilde{u} *}\right)\right.\right. \\
& \left.\times\left(t_{\beta}^{2} A_{q, j}^{d *} V_{\mathrm{CKM}}^{l, q} m_{d_{q}} R_{m, l}^{\tilde{u}} R_{n, 3+j}^{\tilde{d} *}+A_{q, l}^{u} V_{\mathrm{CKM}}^{q, j} m_{u_{q}} R_{m, 3+l}^{\tilde{u}} R_{n, j}^{\tilde{d} *}\right)\right] \\
& +\sum_{p}^{3}\left[V _ { \mathrm { CKM } } ^ { k , i * } \left\{R_{n, i}^{\tilde{d}}\left(\left(-M_{W}^{2} s_{2 \beta} t_{\beta}+m_{u_{k}}^{2}+t_{\beta}^{2} m_{d_{i}}^{2}\right) R_{m, k}^{\tilde{u} *}+\mu t_{\beta} m_{u_{k}} R_{m, 3+k}^{\tilde{u} *}\right)\right.\right. \\
& \left.+m_{d_{i}} R_{n, 3+i}^{\tilde{d}}\left(\mu^{*} t_{\beta} R_{m, k}^{\tilde{u} *}+\left(1+t_{\beta}^{2}\right) m_{u_{k}} R_{m, 3+k}^{\tilde{u} *}\right)\right\} \\
& \times\left\{A_{p, l}^{u} V_{\mathrm{CKM}}^{p, j} m_{u_{p}} R_{m, 3+l}^{\tilde{u}} R_{n, j}^{\tilde{d} *}+t_{\beta}^{2} A_{p, j}^{d *} V_{\mathrm{CKM}}^{l, p} m_{d_{p}} R_{m, l}^{\tilde{u}} R_{n, 3+j}^{\tilde{d} *}\right\} \\
& +V_{\mathrm{CKM}}^{l, j}\left\{R_{m, l}^{\tilde{u}}\left(\left(-M_{W}^{2} s_{2 \beta} t_{\beta}+m_{u_{l}}^{2}+t_{\beta}^{2} m_{d_{j}}^{2}\right) R_{n, j}^{\tilde{d} *}+\mu t_{\beta} m_{d_{j}} R_{n, 3+j}^{\tilde{d} *}\right)\right. \\
& \left.+m_{u_{l}} R_{m, 3+l}^{\tilde{u}}\left(\mu^{*} t_{\beta} R_{n, j}^{\tilde{d} *}+\left(1+t_{\beta}^{2}\right) m_{d_{j}} R_{n, 3+j}^{\tilde{d} *}\right)\right\} \\
& \left.\times\left\{A_{p, k}^{u *} V_{\mathrm{CKM}}^{p, i} m_{u_{p}} R_{n, i}^{\tilde{d}} R_{m, 3+k}^{\tilde{u} *}+t_{\beta}^{2} A_{p, i}^{d} V_{\mathrm{CKM}}^{k, p *} m_{d_{p}} R_{n, 3+i}^{\tilde{d}} R_{m, k}^{\tilde{u} *}\right\}\right] \\
& +\left[V _ { \mathrm { CKM } } ^ { l , j } V _ { \mathrm { CKM } } ^ { k , i * } \left\{R_{n, i}^{\tilde{d}}\left(\left(-M_{W}^{2} s_{2 \beta} t_{\beta}+m_{u_{k}}^{2}+t_{\beta}^{2} m_{d_{i}}^{2}\right) R_{m, k}^{\tilde{u} *}+\mu t_{\beta} m_{u_{k}} R_{m, 3+k}^{\tilde{u} *}\right)\right.\right. \\
& \left.+m_{d_{i}} R_{n, 3+i}^{\tilde{d}}\left(\mu^{*} t_{\beta} R_{m, k}^{\tilde{u} *}+\left(1+t_{\beta}^{2}\right) m_{u_{k}} R_{m, 3+k}^{\tilde{u} *}\right)\right\} \\
& \times\left\{R_{m, l}^{\tilde{u}}\left(\left(-M_{W}^{2} s_{2 \beta} t_{\beta}+m_{u_{l}}^{2}+t_{\beta}^{2} m_{d_{j}}^{2}\right) R_{n, j}^{\tilde{d} *}+\mu t_{\beta} m_{d_{j}} R_{n, 3+j}^{\tilde{d *}}\right)\right. \\
& \left.\left.\left.+m_{u_{l}} R_{m, 3+l}^{\tilde{u}}\left(\mu^{*} t_{\beta} R_{n, j}^{\tilde{d} *}+\left(1+t_{\beta}^{2}\right) m_{d_{j}} R_{n, 3+j}^{\tilde{d} *}\right)\right\}\right]\right\} \\
& \Sigma_{H^{-} H^{+}}^{1 \tilde{q}}=\sum_{l=1}^{6} \sum_{i=1}^{3} \frac{1}{16 c_{W}^{2} M_{W}^{2} \pi t_{\beta}^{2} s_{W}^{2}} \alpha A_{0}\left[m_{\tilde{u}_{l}}^{2}\right]\left\{2 R_{l, 3+i}^{\tilde{u}} R_{l, 3+i}^{\tilde{u} *}\left(-2 c_{2 \beta} M_{W}^{2} t_{\beta}^{2} s_{W}^{2}+3 c_{W}^{2} m_{u_{i}}^{2}\right)\right. \\
& \left.+t_{\beta}^{2} R_{l, i}^{\tilde{u}}\left(R_{l, i}^{\tilde{u} *} c_{2 \beta} m_{w}^{2}\left(1+2 c_{W}^{2}\right)+\sum_{j=1}^{3} \sum_{k=1}^{3} 6 R_{l, j}^{\tilde{u} *} c_{W}^{2} t_{\beta}^{2} V_{\mathrm{CKM}}^{i, k} V_{\mathrm{CKM}}^{j, k *} m_{d_{k}}^{2}\right)\right\}
\end{aligned}
$$




$$
\begin{aligned}
& +\sum_{l=1}^{6} \sum_{i=1}^{3} \frac{1}{16 c_{W}^{2} M_{W}^{2} \pi t_{\beta}^{2} s_{W}^{2}} \alpha A_{0}\left[m_{\tilde{d}_{l}}^{2}\right]\left\{2 R_{l, 3+i}^{\tilde{d}} R_{l, 3+i}^{\tilde{d} *}\left(c_{2 \beta} M_{W}^{2} t_{\beta}^{2} s_{W}^{2}+3 c_{W}^{2} t_{\beta}^{4} m_{d_{i}}^{2}\right)\right. \\
& \left.+R_{l, i}^{\tilde{d}}\left(R_{l, i}^{\tilde{d} *} c_{2 \beta} m_{w}^{2} t_{\beta}^{2}\left(1-4 c_{W}^{2}\right)+\sum_{j=1}^{3} \sum_{k=1}^{3} 6 R_{l, j}^{\tilde{d} *} c_{W}^{2} V_{\mathrm{CKM}}^{k, j} V_{\mathrm{CKM}}^{k, i *} m_{u_{k}}^{2}\right)\right\}
\end{aligned}
$$

- $Z$

$$
\begin{aligned}
& \Sigma_{Z Z}^{2 q}=-\sum_{i=1}^{3} \frac{\left(9-24 s_{W}^{2}+32 s_{W}^{4}\right) \alpha}{36 c_{W}^{2} \pi s_{W}^{2}}\left\{A_{0}\left[m_{u_{i}}^{2}\right]+p^{2} B_{1}\left[p^{2}, m_{u_{i}}^{2}, m_{u_{i}}^{2}\right]\right\} \\
& -\sum_{i=1}^{3} \frac{\left(9+48 s_{W}^{2}-64 s_{W}^{4}\right) \alpha}{72 c_{W}^{2} \pi s_{W}^{2}}\left\{m_{u_{i}}^{2} B_{0}\left[p^{2}, m_{u_{i}}^{2}, m_{u_{i}}^{2}\right]\right\} \\
& -\sum_{i=1}^{3} \frac{\left(9-12 s_{W}^{2}+8 s_{W}^{4}\right) \alpha}{36 c_{W}^{2} \pi s_{W}^{2}}\left\{A_{0}\left[m_{d_{i}}^{2}\right]+p^{2} B_{1}\left[p^{2}, m_{d_{i}}^{2}, m_{d_{i}}^{2}\right]\right\} \\
& -\sum_{i=1}^{3} \frac{\left(9+24 s_{W}^{2}-16 s_{W}^{4}\right) \alpha}{72 c_{W}^{2} \pi s_{W}^{2}}\left\{m_{d_{i}}^{2} B_{0}\left[p^{2}, m_{d_{i}}^{2}, m_{d_{i}}^{2}\right]\right\} \\
& \Sigma_{Z Z}^{2 \tilde{q}}=-\sum_{m, n}^{6} \sum_{i, j}^{3} \frac{\alpha}{72 c_{W}^{2} \pi s_{W}^{2}}\left\{A_{0}\left[m_{\tilde{u}_{n}}^{2}\right]+2 m_{\tilde{u}_{m}}^{2} B_{0}\left[p^{2}, m_{\tilde{u}_{m}}^{2}, m_{\tilde{u}_{n}}^{2}\right]\right. \\
& \left.+\left(p^{2}+m_{\tilde{u}_{m}}^{2}-m_{\tilde{u}_{n}}^{2}\right) B_{1}\left[p^{2}, m_{\tilde{u}_{m}}^{2}, m_{\tilde{u}_{n}}^{2}\right]-\frac{p^{2}}{3}+m_{\tilde{u}_{m}}^{2}-m_{\tilde{u}_{n}}^{2}\right\} \\
& \times\left\{\left(-3+4 s_{W}^{2}\right) R_{n, i}^{\tilde{u}} R_{m, i}^{\tilde{u} *}+4 s_{W}^{2} R_{n, 3+i}^{\tilde{u}} R_{m, 3+i}^{\tilde{u} *}\right\} \\
& \left\{\left(-3+4 s_{W}^{2}\right) R_{m, j}^{\tilde{u}} R_{n, j}^{\tilde{u} *}+4 s_{W}^{2} R_{m, 3+j}^{\tilde{u}} R_{n, 3+j}^{\tilde{u} *}\right\} \\
& -\sum_{m, n}^{6} \sum_{i, j}^{3} \frac{\alpha}{72 c_{W}^{2} \pi s_{W}^{2}}\left\{A_{0}\left[m_{\tilde{d}_{n}}^{2}\right]+2 m_{\tilde{d}_{m}}^{2} B_{0}\left[p^{2}, m_{\tilde{d}_{m}}^{2}, m_{\tilde{d}_{n}}^{2}\right]\right. \\
& \left.+\left(p^{2}+m_{\tilde{d}_{m}}^{2}-m_{\tilde{d}_{n}}^{2}\right) B_{1}\left[p^{2}, m_{\tilde{d}_{m}}^{2}, m_{\tilde{d}_{n}}^{2}\right]-\frac{p^{2}}{3}+m_{\tilde{d}_{m}}^{2}-m_{\tilde{d}_{n}}^{2}\right\} \\
& \times\left\{\left(-3+2 s_{W}^{2}\right) R_{n, i}^{\tilde{d}} R_{m, i}^{\tilde{d} *}+2 s_{W}^{2} R_{n, 3+i}^{\tilde{d}} R_{m, 3+i}^{\tilde{d} *}\right\} \\
& \left\{\left(-3+2 s_{W}^{2}\right) R_{m, j}^{\tilde{d}} R_{n, j}^{\tilde{d} *}+2 s_{W}^{2} R_{m, 3+j}^{\tilde{d}} R_{n, 3+j}^{\tilde{d} *}\right\} \\
& \Sigma_{Z Z}^{1 \tilde{q}}=\sum_{l=1}^{6} \sum_{i=1}^{3} \frac{1}{24 c_{W}^{2} \pi s_{W}^{2}} \alpha A_{0}\left[m_{\tilde{u}_{l}}^{2}\right]\left(R_{l, i}^{\tilde{u}} R_{l, i}^{\tilde{u} *}\left(-3+4 s_{W}^{2}\right)^{2}+16 s_{W}^{4} R_{l, 3+i}^{\tilde{u}} R_{l, 3+i}^{\tilde{u} *}\right) \\
& +\sum_{l=1}^{6} \sum_{i=1}^{3} \frac{1}{24 c_{W}^{2} \pi s_{W}^{2}} \alpha A_{0}\left[m_{\tilde{d}_{l}}^{2}\right]\left(R_{l, i}^{\tilde{d}} R_{l, i}^{\tilde{d} *}\left(3-2 s_{W}^{2}\right)^{2}+4 s_{W}^{4} R_{l, 3+i}^{\tilde{d}} R_{l, 3+i}^{\tilde{d} *}\right)
\end{aligned}
$$

- $W$

$$
\Sigma_{W W}^{2 q}=-\sum_{i, l}^{3} \frac{\alpha}{4 \pi s_{W}^{2}} V_{\mathrm{CKM}}^{l, i} V_{\mathrm{CKM}}^{l, i *}\left\{2 A_{0}\left[m_{d_{i}}^{2}\right]+m_{u_{l}}^{2} B_{0}\left[p^{2}, m_{d_{i}}^{2}, m_{u_{l}}^{2}\right]\right.
$$




$$
\begin{aligned}
& \left.+\left(m_{d_{l}}^{2}-m_{u_{l}}^{2}+2 p^{2}\right) B_{1}\left[p^{2}, m_{u_{i}}^{2}, m_{d_{l}}^{2}\right]\right\} \\
\Sigma_{W W}^{2 \tilde{q}}= & -\sum_{m, n}^{6} \sum_{i, j, k, l}^{3} \frac{3 \alpha}{12 \pi s_{W}^{2}} V_{\mathrm{CKM}}^{k, i} V_{\mathrm{CKM}}^{l, j *} R_{m, k}^{\tilde{u}} R_{m, l}^{\tilde{u} *} R_{n, j}^{\tilde{d}} R_{n, i}^{\tilde{d} *}\left\{A_{0}\left[m_{\tilde{d}_{n}}^{2}\right]\right. \\
& +2 m_{\tilde{u}_{m}}^{2} B_{0}\left[p^{2}, m_{\tilde{u}_{m}}^{2}, m_{\tilde{d}_{n}}^{2}\right] \\
& \left.+\left(p^{2}+m_{\tilde{u}_{m}}^{2}-m_{\tilde{d}_{n}}^{2}\right) B_{1}\left[p^{2}, m_{\tilde{u}_{m}}^{2}, m_{\tilde{d}_{n}}^{2}\right]-\frac{p^{2}}{3}+m_{\tilde{u}_{m}}^{2}+m_{\tilde{d}_{n}}^{2}\right\} \\
\Sigma_{W W}^{1 \tilde{q}}= & \sum_{l=1}^{6} \sum_{i=1}^{3} \frac{3}{8 \pi s_{W}^{2}} \alpha A_{0}\left[m_{\tilde{u}_{l}}^{2}\right] R_{l, i}^{\tilde{u}} R_{l, i}^{\tilde{u} *}+\sum_{l=1}^{6} \sum_{i=1}^{3} \frac{3}{8 \pi s_{W}^{2}} \alpha A_{0}\left[m_{\tilde{d}_{l}}^{2}\right] R_{l, i}^{\tilde{d}} R_{l, i}^{\tilde{d} *}
\end{aligned}
$$

- Tadpoles

$$
\begin{aligned}
& T_{h}^{q}=-\sum_{i}^{3} \frac{3}{8 \pi^{2} M_{W} s_{\beta} s_{W}} c_{\alpha} m_{u_{i}}^{2} e A_{0}\left[m_{u_{i}}^{2}\right] \\
& T_{h}^{\tilde{q}}=\sum_{m}^{6} \sum_{i, j}^{3} \frac{1}{32 \pi^{2} c_{W} M_{W} s_{\beta} s_{W}} e A_{0}\left[m_{\tilde{u}_{m}}^{2}\right] \\
& \times\left[\left\{\delta_{i, j}\left(M_{W} m_{Z} s_{\alpha+\beta} s_{\beta}\left(-3+4 s_{W}^{2}\right)+6 c_{\alpha} c_{W} m_{u_{i}}^{2}\right) R_{m, j}^{\tilde{u}}\right.\right. \\
& \left.+3 c_{W}\left(c_{\alpha} A_{i, j}^{u}+\mu^{*} s_{\alpha} \delta_{i, j}\right) m_{u_{i}} R_{m, 3+j}^{\tilde{u}}\right\} R_{m, i}^{\tilde{u} *} \\
& +\left\{3 c_{\alpha} c_{W} A_{j, i}^{u *} m_{u_{j}} R_{m, j}^{\tilde{u}}+3 c_{W} \mu s_{\alpha} \delta_{i, j} m_{u_{i}} R_{m, j}^{\tilde{u}}\right. \\
& \left.\left.+2 \delta_{i, j}\left(-2 M_{W} m_{Z} s_{\alpha+\beta} s_{\beta} s_{W}^{2}+3 c_{\alpha} c_{W} m_{u_{i}}^{2}\right) R_{m, 3+j}^{\tilde{u}}\right\} R_{m, 3+i}^{\tilde{u} *}\right] \\
& +\sum_{i}^{3} \frac{3}{8 \pi^{2} M_{W} c_{\beta} s_{W}} s_{\alpha} m_{d_{i}}^{2} e A_{0}\left[m_{d_{i}}^{2}\right]-\sum_{m}^{6} \sum_{i, j}^{3} \frac{1}{32 \pi^{2} c_{W} M_{W} c_{\beta} s_{W}} e A_{0}\left[m_{\tilde{d}_{m}}^{2}\right] \\
& \times\left[\left\{\delta_{i, j}\left(M_{W} m_{Z} s_{\alpha+\beta} c_{\beta}\left(-3+2 s_{W}^{2}\right)+6 s_{\alpha} c_{W} m_{d_{i}}^{2}\right) R_{m, j}^{\tilde{d}}\right.\right. \\
& \left.+3 c_{W}\left(s_{\alpha} A_{i, j}^{d}+\mu^{*} c_{\alpha} \delta_{i, j}\right) m_{d_{i}} R_{m, 3+j}^{\tilde{d}}\right\} R_{m, i}^{\tilde{d} *} \\
& +\left\{3 s_{\alpha} c_{W} A_{j, i}^{d *} m_{d_{j}} R_{m, j}^{\tilde{d}}+3 c_{W} \mu c_{\alpha} \delta_{i, j} m_{d_{i}} R_{m, j}^{\tilde{d}}\right. \\
& \left.\left.-2 \delta_{i, j}\left(M_{W} m_{Z} s_{\alpha+\beta} c_{\beta} s_{W}^{2}-3 s_{\alpha} c_{W} m_{d_{i}}^{2}\right) R_{m, 3+j}^{\tilde{d}}\right\} R_{m, 3+i}^{\tilde{d} *}\right]
\end{aligned}
$$


The self-energies for $T_{H}$ are obtained using the replacements of eq. (A.1) on the results of $T_{h}$.

\section{References}

[1] H.P. Nilles, Supersymmetry, supergravity and particle physics, Phys. Rept. 110 (1984) 1 [INSPIRE].

[2] H.E. Haber and G.L. Kane, The search for supersymmetry: probing physics beyond the Standard Model, Phys. Rept. 117 (1985) 75 [INSPIRE].

[3] R. Barbieri, Looking beyond the Standard Model: the supersymmetric option, Riv. Nuovo Cim. 11 (1988) 1.

[4] S. Heinemeyer, MSSM Higgs physics at higher orders, Int. J. Mod. Phys. A 21 (2006) 2659 [hep-ph/0407244] [INSPIRE].

[5] A. Djouadi, The anatomy of electro-weak symmetry breaking. II. The Higgs bosons in the minimal supersymmetric model, Phys. Rept. 459 (2008) 1 [hep-ph/0503173] [INSPIRE].

[6] W. Ehrenfeld, SUSY searches at ATLAS, talk given at SUSY11, https://indico.fnal.gov/ contributionDisplay.py?sessionId $=10 \&$ contribId $=257 \&$ confId $=3563$, Fermilab, Batavia U.S.A. August 2011.

[7] I. Melzer-Pellmann, SUSY searches at CMS, talk given at SUSY11, https://indico.fnal.gov/ contributionDisplay.py?sessionId $=10 \&$ contribId $=258 \&$ confId $=3563$, Fermilab, Batavia U.S.A. August 2011.

[8] Particle Data Group collaboration, K. Nakamura et al., Review of particle physics, J. Phys. G 37 (2010) 075021 [INSPIRE].

[9] LeP Working Group for Higgs boson searches, AlEPH, DELPHI, L3 and OPAL collaborations, R. Barate et al., Search for the Standard Model Higgs boson at LEP, Phys. Lett. B 565 (2003) 61 [hep-ex/0306033] [INSPIRE].

[10] AlEPH, DELPhi, L3, OPAL and LEP Working Group for Higgs Boson Searches collaborations, S. Schael et al., Search for neutral MSSM Higgs bosons at LEP, Eur. Phys. J. C 47 (2006) 547 [hep-ex/0602042] [INSPIRE].

[11] Tevatron new phenomena $\&$ Higgs working group webpage, http://tevnphwg.fnal.gov/.

[12] M. Vazquez Acosta, Higgs searches at the LHC, talk given at SUSY11, https://indico. fnal.gov/contributionDisplay.py?sessionId=13\&contribId=261\&confId=3563, Fermilab, Batavia U.S.A. August 2011.

[13] ATLAS collaboration, G. Aad et al., Expected performance of the ATLAS experiment detector, trigger and physics, arXiv:0901.0512 [INSPIRE].

[14] CMS collaboration, G. Bayatian et al., CMS technical design report, volume II: Physics performance, J. Phys. G 34 (2007) 995 [INSPIRE].

[15] V. Büscher and K. Jakobs, Higgs boson searches at hadron colliders, Int. J. Mod. Phys. A 20 (2005) 2523 [hep-ph/0504099] [inSPIRE].

[16] ECFA/DESY LC Physics Working Group collaboration, J. Aguilar-Saavedra et al., TESLA: the superconducting electron positron linear collider with an integrated $x$-ray laser laboratory. TDR part 3. Physics at an $e^{+} e^{-}$linear collider, hep-ph/0106315 [INSPIRE]. 
[17] TESLA - the superconducting electron-positron linear collider with an integrated X-ray laser laboratory technical design report webpage,

http://tesla.desy.de/new_pages/TDR_CD/start.html.

[18] K. Ackermann et al., Extended joint ECFA/DESY study on physics and detectors for a linear electron-positron collider, prepared for $4^{\text {th }}$ ECFA/DESY Workshop on Physics and Detectors for a $90 \mathrm{GeV}$ to $800 \mathrm{GeV}$ Linear $e^{+} e^{-}$Collider, DESY-PROC-2004-01, Amsterdam The Netherlands April 1-4 2003 [INSPIRE].

[19] S. Heinemeyer et al., Toward high precision Higgs-boson measurements at the international linear $e^{+} e^{-}$collider, hep-ph/0511332 [INSPIRE].

[20] LHC/LC Study Group collaboration, G. Weiglein et al., Physics interplay of the LHC and the ILC, Phys. Rept. 426 (2006) 47 [hep-ph/0410364] [INSPIRE].

[21] A. De Roeck et al., From the LHC to future colliders, Eur. Phys. J. C 66 (2010) 525 [arXiv:0909.3240] [INSPIRE].

[22] S. Heinemeyer, W. Hollik, H. Rzehak and G. Weiglein, The Higgs sector of the complex MSSM at two-loop order: QCD contributions, Phys. Lett. B 652 (2007) 300 [arXiv:0705.0746] [INSPIRE].

[23] M. Frank et al., The Higgs boson masses and mixings of the complex MSSM in the Feynman-diagrammatic approach, JHEP 02 (2007) 047 [hep-ph/0611326] [INSPIRE].

[24] G. Degrassi, S. Heinemeyer, W. Hollik, P. Slavich and G. Weiglein, Towards high precision predictions for the MSSM Higgs sector, Eur. Phys. J. C 28 (2003) 133 [hep-ph/0212020] [INSPIRE].

[25] S.P. Martin, Three-loop corrections to the lightest Higgs scalar boson mass in supersymmetry, Phys. Rev. D 75 (2007) 055005 [hep-ph/0701051] [INSPIRE].

[26] R.V. Harlander, P. Kant, L. Mihaila and M. Steinhauser, Higgs boson mass in supersymmetry to three loops, Phys. Rev. Lett. 100 (2008) 191602 [Erratum ibid. 101 (2008) 039901] [arXiv:0803.0672] [INSPIRE].

[27] P. Kant, R. Harlander, L. Mihaila and M. Steinhauser, Light MSSM Higgs boson mass to three-loop accuracy, JHEP 08 (2010) 104 [arXiv: 1005.5709] [INSPIRE].

[28] S. Heinemeyer, W. Hollik, F. Merz and S. Peñaranda, Electroweak precision observables in the MSSM with Non-Minimal Flavor Violation, Eur. Phys. J. C 37 (2004) 481 [hep-ph/0403228] [INSPIRE].

[29] S. Heinemeyer, W. Hollik and G. Weiglein, FeynHiggs: a program for the calculation of the masses of the neutral CP even Higgs bosons in the MSSM,

Comput. Phys. Commun. 124 (2000) 76 [hep-ph/9812320] [INSPIRE].

[30] FeynHiggs webpage, http://www.feynhiggs.de/.

[31] S. Heinemeyer, W. Hollik and G. Weiglein, The masses of the neutral CP-even Higgs bosons in the MSSM: accurate analysis at the two loop level, Eur. Phys. J. C 9 (1999) 343 [hep-ph/9812472] [INSPIRE].

[32] J. Guasch and J. Solà, FCNC top quark decays: a door to SUSY physics in high luminosity colliders?, Nucl. Phys. B 562 (1999) 3 [hep-ph/9906268] [INSPIRE].

[33] S. Bejar, F. Dilme, J. Guasch and J. Solà, Higgs boson flavor changing neutral decays into bottom quarks in supersymmetry, JHEP 08 (2004) 018 [hep-ph/0402188] [INSPIRE]. 
[34] A.M. Curiel, M.J. Herrero and D. Temes, Flavor changing neutral Higgs boson decays from squark Gluino loops, Phys. Rev. D 67 (2003) 075008 [hep-ph/0210335] [INSPIRE].

[35] A. Curiel, M. Herrero, W. Hollik, F. Merz and S. Peñaranda, SUSY electroweak one loop contributions to flavor changing Higgs-Boson decays, Phys. Rev. D 69 (2004) 075009 [hep-ph/0312135] [INSPIRE].

[36] T. Hahn, W. Hollik, J. Illana and S. Peñaranda, Interplay between $H \rightarrow b \bar{s}$ and $b \rightarrow s \gamma$ in the MSSM with Non-Minimal Flavor Violation, hep-ph/0512315 [INSPIRE].

[37] J. Cao, G. Eilam, K.-I. Hikasa and J.M. Yang, Experimental constraints on stop-scharm flavor mixing and implications in top-quark FCNC processes, Phys. Rev. D 74 (2006) 031701 [hep-ph/0604163] [INSPIRE].

[38] S. Dittmaier, G. Hiller, T. Plehn and M. Spannowsky, Charged-Higgs collider signals with or without flavor, Phys. Rev. D 77 (2008) 115001 [arXiv:0708.0940] [INSPIRE].

[39] J. Cao et al., SUSY-induced FCNC top-quark processes at the Large Hadron Collider, Phys. Rev. D 75 (2007) 075021 [hep-ph/0702264] [INSPIRE].

[40] G. Degrassi, P. Gambino and P. Slavich, SusyBSG: a Fortran code for $B R\left(B \rightarrow X_{s} \gamma\right)$ in the MSSM with Minimal Flavor Violation, Comput. Phys. Commun. 179 (2008) 759 [arXiv: 0712.3265] [INSPIRE].

[41] G. Degrassi, P. Gambino and P. Slavich, QCD corrections to radiative $B$ decays in the MSSM with Minimal Flavor Violation, Phys. Lett. B 635 (2006) 335 [hep-ph/0601135] [INSPIRE].

[42] G. Isidori and P. Paradisi, Hints of large $\tan \beta$ in flavour physics, Phys. Lett. B 639 (2006) 499 [hep-ph/0605012] [INSPIRE].

[43] G. Isidori, F. Mescia, P. Paradisi and D. Temes, Flavour physics at large $\tan \beta$ with a Bino-like LSP, Phys. Rev. D 75 (2007) 115019 [hep-ph/0703035] [INSPIRE].

[44] S. Baek, T. Goto, Y. Okada and K.-I. Okumura, Muon anomalous magnetic moment, lepton flavor violation and flavor changing neutral current processes in SUSY GUT with right-handed neutrino, Phys. Rev. D 64 (2001) 095001 [hep-ph/0104146] [INSPIRE].

[45] J. Foster, K.-I. Okumura and L. Roszkowski, Probing the flavor structure of supersymmetry breaking with rare B-processes: a beyond leading order analysis, JHEP 08 (2005) 094 [hep-ph/0506146] [INSPIRE].

[46] F. Gabbiani, E. Gabrielli, A. Masiero and L. Silvestrini, A complete analysis of FCNC and $C P$ constraints in general SUSY extensions of the Standard Model, Nucl. Phys. B 477 (1996) 321 [hep-ph/9604387] [inSPIRE].

[47] D. Becirevic et al., $B_{d}-\bar{B}_{d}$ mixing and the $B_{d} \rightarrow J / \psi K_{s}$ asymmetry in general SUSY models, Nucl. Phys. B 634 (2002) 105 [hep-ph/0112303] [INSPIRE].

[48] L.J. Hall, V.A. Kostelecky and S. Raby, New flavor violations in supergravity models, Nucl. Phys. B 267 (1986) 415 [inSPIRE].

[49] J. Gunion and H.E. Haber, Higgs bosons in supersymmetric models. 1, Nucl. Phys. B 272 (1986) 1 [Erratum ibid. B 402 (1993) 567] [InSPIRE].

[50] J. Küblbeck, M. Böhm and A. Denner, FeynArts: computer algebraic generation of Feynman graphs and amplitudes, Comput. Phys. Commun. 60 (1990) 165 [INSPIRE].

[51] T. Hahn, Generating Feynman diagrams and amplitudes with FeynArts 3, Comput. Phys. Commun. 140 (2001) 418 [hep-ph/0012260] [InSPIRE]. 
[52] The FeynArts website, http://www.feynarts.de/.

[53] T. Hahn and M. Pérez-Victoria, Automatized one loop calculations in four-dimensions and D-dimensions, Comput. Phys. Commun. 118 (1999) 153 [hep-ph/9807565] [INSPIRE].

[54] T. Hahn and C. Schappacher, The implementation of the minimal supersymmetric Standard Model in FeynArts and FormCalc, Comput. Phys. Commun. 143 (2002) 54 [hep-ph/0105349] [INSPIRE].

[55] P. Gambino and M. Misiak, Quark mass effects in $\bar{B} \rightarrow X_{s} \gamma$, Nucl. Phys. B 611 (2001) 338 [hep-ph/0104034] [INSPIRE].

[56] S. AbdusSalam et al., Benchmark models, planes, lines and points for future SUSY searches at the LHC, Eur. Phys. J. C 71 (2011) 1835 [arXiv:1109.3859] [InSPIRE].

[57] W. Porod and F. Staub, SPheno 3.1: extensions including flavour, CP-phases and models beyond the MSSM, arXiv:1104.1573 [INSPIRE].

[58] B. Allanach et al., The Snowmass points and slopes: benchmarks for SUSY searches, Eur. Phys. J. C 25 (2002) 113 [hep-ph/0202233] [INSPIRE].

[59] O. Buchmueller et al., Supersymmetry and Dark Matter in light of LHC 2010 and Xenon100 data, Eur. Phys. J. C 71 (2011) 1722 [arXiv:1106.2529] [INSPIRE].

[60] S. Bertolini, F. Borzumati, A. Masiero and G. Ridolfi, Effects of supergravity induced electroweak breaking on rare B decays and mixings, Nucl. Phys. B 353 (1991) 591 [INSPIRE].

[61] P.L. Cho, M. Misiak and D. Wyler, $K_{L} \rightarrow \pi^{0} e^{+} e^{-}$and $B \rightarrow X_{s} \ell^{+} \ell^{-}$decay in the MSSM, Phys. Rev. D 54 (1996) 3329 [hep-ph/9601360] [INSPIRE].

[62] G. Degrassi, P. Gambino and G. Giudice, $B \rightarrow X_{s} \gamma$ in supersymmetry: large contributions beyond the leading order, JHEP 12 (2000) 009 [hep-ph/0009337] [INSPIRE].

[63] T. Hurth, E. Lunghi and W. Porod, Untagged $\bar{B} \rightarrow X_{s+d} \gamma C P$ asymmetry as a probe for new physics, Nucl. Phys. B 704 (2005) 56 [hep-ph/0312260] [INSPIRE].

[64] M. Ciuchini, G. Degrassi, P. Gambino and G. Giudice, Next-to-leading QCD corrections to $B \rightarrow X_{s} \gamma$ : Standard Model and two Higgs doublet model, Nucl. Phys. B 527 (1998) 21 [hep-ph/9710335] [INSPIRE].

[65] L.J. Hall, R. Rattazzi and U. Sarid, The top quark mass in supersymmetric $\mathrm{SO}(10)$ unification, Phys. Rev. D 50 (1994) 7048 [hep-ph/9306309] [INSPIRE].

[66] M.S. Carena, D. Garcia, U. Nierste and C.E. Wagner, $b \rightarrow s \gamma$ and supersymmetry with large $\tan \beta$, Phys. Lett. B 499 (2001) 141 [hep-ph/0010003] [INSPIRE].

[67] M.S. Carena, D. Garcia, U. Nierste and C.E. Wagner, Effective Lagrangian for the $\bar{t} b H^{+}$ interaction in the MSSM and charged Higgs phenomenology, Nucl. Phys. B 577 (2000) 88 [hep-ph/9912516] [INSPIRE].

[68] G. Isidori and A. Retico, Scalar flavor changing neutral currents in the large $\tan \beta$ limit, JHEP 11 (2001) 001 [hep-ph/0110121] [INSPIRE].

[69] A.J. Buras, P.H. Chankowski, J. Rosiek and L. Slawianowska, $\Delta M_{d, s}, B^{0} d, s \rightarrow \mu^{+} \mu^{-}$and $B \rightarrow X_{s} \gamma$ in supersymmetry at large $\tan \beta$, Nucl. Phys. B 659 (2003) 3 [hep-ph/0210145] [INSPIRE].

[70] G. Isidori and A. Retico, $B_{s, d} \rightarrow \ell^{+} \ell^{-}$and $K_{L} \rightarrow \ell^{+} \ell^{-}$in SUSY models with nonminimal sources of flavor mixing, JHEP 09 (2002) 063 [hep-ph/0208159] [INSPIRE]. 
[71] A. Crivellin and U. Nierste, Chirally enhanced corrections to FCNC processes in the generic MSSM, Phys. Rev. D 81 (2010) 095007 [arXiv:0908.4404] [INSPIRE].

[72] A. Crivellin and U. Nierste, Supersymmetric renormalisation of the CKM matrix and new constraints on the squark mass matrices, Phys. Rev. D 79 (2009) 035018 [arXiv:0810.1613] [INSPIRE].

[73] K.-I. Okumura and L. Roszkowski, Weakened Constraints from $b \rightarrow s \gamma$ on supersymmetry flavor mixing due to next-to-leading-order corrections, Phys. Rev. Lett. 92 (2004) 161801 [hep-ph/0208101] [INSPIRE].

[74] K.-I. Okumura and L. Roszkowski, Large beyond leading order effects in $b \rightarrow s \gamma$ in supersymmetry with general flavor mixing, JHEP 10 (2003) 024 [hep-ph/0308102] [INSPIRE].

[75] J. Foster, K.-I. Okumura and L. Roszkowski, New Higgs effects in B-physics in supersymmetry with general flavour mixing, Phys. Lett. B 609 (2005) 102 [hep-ph/0410323] [INSPIRE].

[76] Heavy Flavor Averaging Group collaboration, D. Asner et al., Averages of b-hadron, c-hadron and $\tau$-lepton properties, arXiv:1010.1589 [INSPIRE].

[77] M. Misiak, $\bar{B} \rightarrow X_{s} \gamma$ : current status, Acta Phys. Polon. B 40 (2009) 2987 [arXiv: 0911.1651] [INSPIRE].

[78] P.H. Chankowski and L. Slawianowska, $B_{d, s}^{0} \rightarrow \mu^{-} \mu^{+}$decay in the MSSM, Phys. Rev. D 63 (2001) 054012 [hep-ph/0008046] [InSPIRE].

[79] C. Bobeth, T. Ewerth, F. Krüger and J. Urban, Enhancement of $B\left(\bar{B}_{d} \rightarrow \mu^{+} \mu^{-)}\right) / B\left(\bar{B}_{s} \rightarrow \mu^{+} \mu^{-}\right)$in the MSSM with Minimal Flavor Violation and large $\tan \beta$, Phys. Rev. D 66 (2002) 074021 [hep-ph/0204225] [INSPIRE].

[80] K. Babu and C.F. Kolda, Higgs mediated $B^{0} \rightarrow \mu^{+} \mu^{-}$in minimal supersymmetry, Phys. Rev. Lett. 84 (2000) 228 [hep-ph/9909476] [INSPIRE].

[81] C. Bobeth, T. Ewerth, F. Krüger and J. Urban, Analysis of neutral Higgs boson contributions to the decays $B_{s} \rightarrow \ell^{+} \ell^{-}$and $\bar{B} \rightarrow K \ell^{+} \ell^{-}$, Phys. Rev. D 64 (2001) 074014 [hep-ph/0104284] [INSPIRE].

[82] CMS and LHCB collaborations, Search for the rare decay $B_{s}^{0} \rightarrow \mu^{+} \mu^{-}$at the LHC with the $C M S$ and LHCb experiments. Combination of LHC results of the search for $B_{s} \rightarrow \mu^{+} \mu^{-}$ decays, CMS-PAS-BPH-11-019, CERN, Geneva Switzerland (2011) [INSPIRE].

[83] A.J. Buras, Flavour theory: 2009, PoS(EPS-HEP 2009) 024 [arXiv:0910.1032] [INSPIRE].

[84] D. Becirevic, V. Giménez, G. Martinelli, M. Papinutto and J. Reyes, $B$ parameters of the complete set of matrix elements of $\Delta B=2$ operators from the lattice, JHEP 04 (2002) 025 [hep-lat/0110091] [INSPIRE].

[85] D. Becirevic, V. Giménez, G. Martinelli, M. Papinutto and J. Reyes, Combined relativistic and static analysis for all $\Delta B=2$ operators, Nucl. Phys. Proc. Suppl. 106 (2002) 385 [hep-lat/0110117] [INSPIRE].

[86] A.J. Buras, M. Jamin and P.H. Weisz, Leading and next-to-leading QCD corrections to $\epsilon$ parameter and $B^{0}-\bar{B}^{0}$ mixing in the presence of a heavy top quark,

Nucl. Phys. B 347 (1990) 491 [INSPIRE].

[87] E. Golowich, J. Hewett, S. Pakvasa, A.A. Petrov and G.K. Yeghiyan, Relating $B_{s}$ mixing and $B_{s} \rightarrow \mu^{+} \mu^{-}$with new physics, Phys. Rev. D 83 (2011) 114017 [arXiv:1102.0009] [INSPIRE]. 
[88] E. Lunghi, Flavor physics in the LHC era: the role of the lattice, talk given at Lattice 2011, http://tsailab.chem.pacific.edu/lat11/plenary/lunghi/lunghi-lattice2011.pdf, July 11-16 2011.

[89] J. Gunion, H. Haber, G. Kane and S. Dawson, The Higgs hunter's guide, Addison-Wesley, Boston U.S.A. (1990).

[90] A. Dabelstein, Fermionic decays of neutral MSSM Higgs bosons at the one loop level, Nucl. Phys. B 456 (1995) 25 [hep-ph/9503443] [INSPIRE].

[91] A. Dabelstein, The one loop renormalization of the MSSM Higgs sector and its application to the neutral scalar Higgs masses, Z. Phys. C 67 (1995) 495 [hep-ph/9409375] [INSPIRE].

[92] A. Denner, Techniques for calculation of electroweak radiative corrections at the one loop level and results for $W$ physics at LEP-200, Fortsch. Phys. 41 (1993) 307 [arXiv:0709.1075] [INSPIRE]. 